\title{
Cross-sections of large-angle hadron production in proton- and pion-nucleus interactions I: beryllium nuclei and beam momenta of $+8.9 \mathrm{GeV} / c$ and $-8.0 \mathrm{GeV} / c$
}

\author{
A. Bolshakova ${ }^{1}$, I. Boyko ${ }^{1}$, G. Chelkov ${ }^{1}$, D. Dedovitch ${ }^{1}$, A. Elagin ${ }^{1,2}$, M. Gostkin ${ }^{1}$, S. Grishin ${ }^{1}$, A. Guskov ${ }^{1}$, \\ Z. Kroumchtein ${ }^{1}$, Yu. Nefedov ${ }^{1}$, K. Nikolaev ${ }^{1}$, A. Zhemchugov ${ }^{1}$, F. Dydak ${ }^{3}$, J. Wotschack ${ }^{3, a}$, A. De Min ${ }^{4, b}$, \\ V. Ammosov ${ }^{5}$, V. Gapienko ${ }^{5}$, V. Koreshev ${ }^{5}$, A. Semak ${ }^{5}$, Yu. Sviridov ${ }^{5}$, E. Usenko ${ }^{5,6}$, V. Zaets ${ }^{5}$ \\ ${ }^{1}$ Joint Institute for Nuclear Research, Dubna, Russia \\ ${ }^{2}$ Present address: Texas A\&M University, College Station, USA \\ ${ }^{3}$ CERN, Geneva, Switzerland \\ ${ }^{4}$ Politecnico di Milano and INFN, Sezione di Milano-Bicocca, Milan, Italy \\ ${ }^{5}$ Institute of High Energy Physics, Protvino, Russia \\ ${ }^{6}$ Present address: Institute for Nuclear Research RAS, Moscow, Russia
}

Received: 23 January 2009 / Revised: 16 April 2009 / Published online: 20 May 2009

(C) Springer-Verlag / Società Italiana di Fisica 2009

\begin{abstract}
We report on double-differential inclusive crosssections of the production of secondary protons, deuterons, and charged pions and kaons, in the interactions with a 5\% $\lambda_{\text {abs }}$ thick stationary beryllium target, of a $+8.9 \mathrm{GeV} / c$ proton and pion beam, and a $-8.0 \mathrm{GeV} / c$ pion beam. Results are given for secondary particles with production angles $20^{\circ}<\theta<125^{\circ}$.
\end{abstract}

PACS 13.85.Ni $\cdot 25.40 . \mathrm{Qa}$

\section{Introduction}

The HARP experiment arose from the realization that the inclusive differential cross-sections of hadron production in the interactions of low-momentum protons with nuclei were known only within a factor of two to three, while more precise cross-sections are in demand for several reasons, notably for the design of the proton driver of a neutrino factory, for the calculation of the atmospheric neutrino flux, for the flux predictions of conventional neutrino beams, and for the modelling of Monte Carlo generators of hadron-nucleus collisions. Consequently, the HARP detector was designed to carry out a programme of systematic and precise measurements of hadron production by protons and pions with momenta from 1.5 to $15 \mathrm{GeV} / c$. It is shown schematically in Fig. 1.

\footnotetext{
a e-mail: joerg.wotschack@cern.ch

${ }^{\mathrm{b}}$ On leave of absence at Ecole Polytechnique Fédérale, Lausanne, Switzerland.
}

The detector extended longitudinally over $14.7 \mathrm{~m}$ and combined a forward spectrometer with a large-angle spectrometer. The latter comprised a cylindrical Time Projection Chamber (TPC) around the target and an array of Resistive Plate Chambers (RPCs) that surrounded the TPC. The purpose of the TPC was track reconstruction and particle identification by $\mathrm{d} E / \mathrm{d} x$. The purpose of the RPCs was to complement the particle identification by time of flight.

The HARP experiment was performed at the CERN Proton Synchrotron in 2001 and 2002 with a set of stationary targets ranging from hydrogen to lead, including beryllium.

We report on the large-angle production (polar angle $\theta$ in the range $20^{\circ}<\theta<125^{\circ}$ ) of secondary protons and charged pions, and of deuterons and charged kaons, in the interactions with a $5 \% \lambda_{\text {abs }}$ Be target of $+8.9 \mathrm{GeV} / c$ protons and pions, and of $-8.0 \mathrm{GeV} / c$ pions.

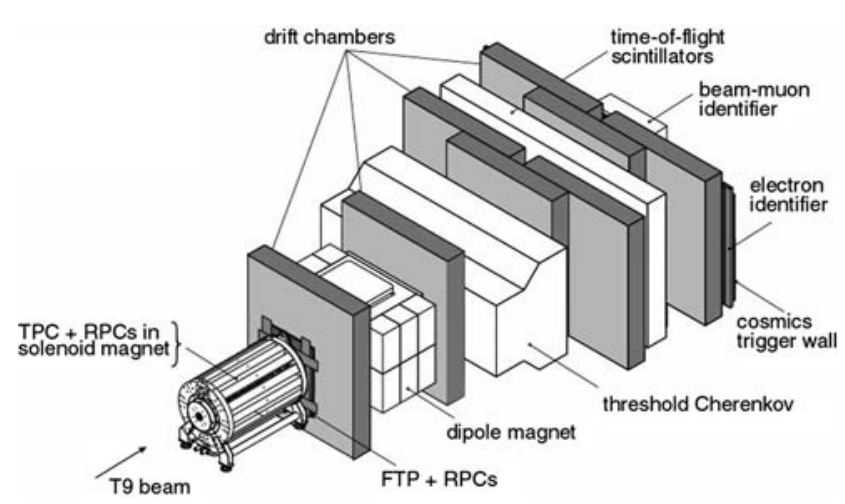

Fig. 1 Schematic view of the HARP detector 
The data analysis presented in this paper rests exclusively on the calibrations of the TPC and the RPCs that we, the HARP-CDP group, published in [1] and [2]. As discussed in [3] and [4], and succinctly summarized in this paper's Appendix, our calibrations disagree with calibrations published by the 'HARP Collaboration' [5-8]. Conclusions of independent review bodies on the discrepancies between our results and those from the HARP Collaboration can be found in $[9,10]$.

\section{The T9 proton and pion beams}

The protons and pions were delivered by the $\mathrm{T} 9$ beam line in the East Hall of CERN's Proton Synchrotron. This beam line supports beam momenta between $1.5 \mathrm{GeV} / c$ and $15 \mathrm{GeV} / c$, with a momentum bite $\Delta p / p \sim 1 \%$.

Beam particle identification was provided for by two threshold Cherenkov counters filled with nitrogen, and by time of flight over a flight path of $24.3 \mathrm{~m}$. In the $+8.9 \mathrm{GeV} / \mathrm{c}$ and $-8.0 \mathrm{GeV} / c$ beams, the pressure of the nitrogen gas was set such that protons were below threshold for Cherenkov light but pions above. The time of flight of each beam particle was measured by three scintillation counters with a precision of 106 ps. ${ }^{1}$

Figure 2(a) shows the relative velocity $\beta$ from the beam time of flight of positive particles in the $+8.9 \mathrm{GeV} / c$ beam, with protons distinguished from 'pions' 2 by the absence of a beam Cherenkov signal. Vice versa, Fig. 2(b) shows the signal charge in one beam Cherenkov counter, with protons and pions distinguished by the signal charge in the other beam Cherenkov counter. All measurements are independent of each other and together permit a clean separation between protons and pions, respectively, with a negligible contamination of less than $0.1 \%$ by the other particle.

The pion beam had a contamination by muons from pion decays. This contamination was measured to be $(1.7 \pm$ $0.5) \%$ of the pion component of the $+8.9 \mathrm{GeV} / c$ beam [11]. For the $-8.0 \mathrm{GeV} / \mathrm{c}$ beam, this contamination is $(1.9 \pm$ $0.5) \%$. The pion beam also had a contamination by electrons from converted photons from $\pi^{0}$ decays. This contamination was determined to be $(1.2 \pm 0.5) \%$ of the pion component of the $+8.9 \mathrm{GeV} / c$ beam [12]. We take the same electron fraction for the $-8.0 \mathrm{GeV} / c$ beam. For the determination of interaction cross-sections of pions, the muon and electron contaminations must be subtracted from the incoming flux of pion-like particles.

\footnotetext{
${ }^{1}$ Under stable conditions of the beam optics, such that an average particle velocity could be used, the time-of-flight precision could be improved to $77 \mathrm{ps}$; in our analysis, no use was made of this option.

${ }^{2}$ The 'pions' comprise small contaminations by muons and electrons, indistinguishable both by time of flight and by beam Cherenkov signals.
}

The beam trajectory was determined by a set of three multiwire proportional chambers (MWPCs), located upstream of the target, several metres apart. The transverse error of the projected impact point on the target was $0.5 \mathrm{~mm}$ from the resolution of the MWPCs, plus a contribution from multiple scattering of the beam particles in various materials. Excluding the target itself, the latter contribution is $0.2 \mathrm{~mm}$ for a $8.9 \mathrm{GeV} / \mathrm{c}$ beam particle.

The size of the beam spot at the position of the target was several millimetres in diameter, determined by the setting
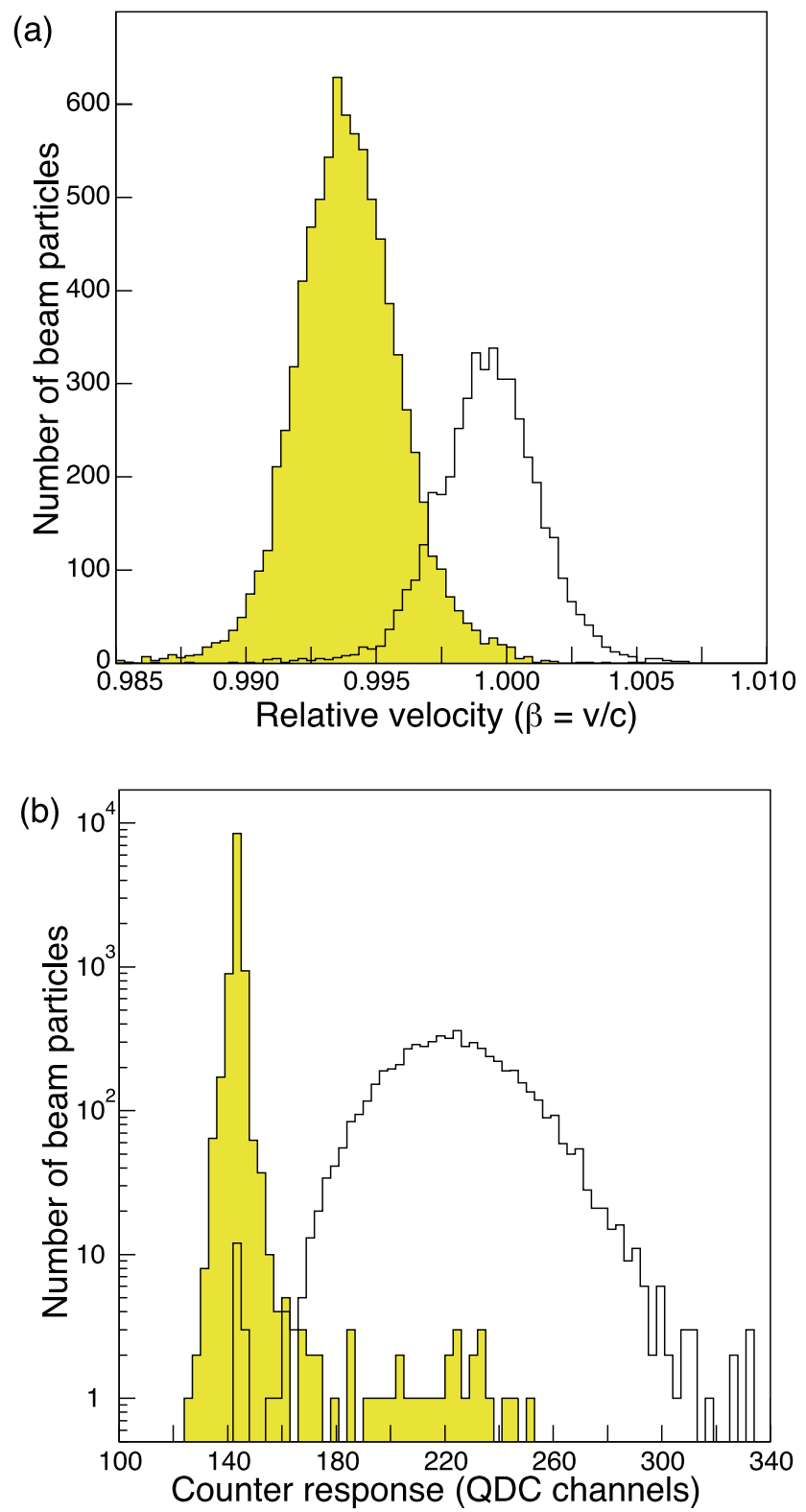

Fig. 2 (a) Relative velocity $\beta$ from the beam time-of-flight system of protons (shaded histogram) and pions, with the particles identified in the beam Cherenkov counters; (b) charge response of the light signal in one beam Cherenkov counter from protons (shaded histogram) and pions, with the particles identified in the other beam Cherenkov counter 

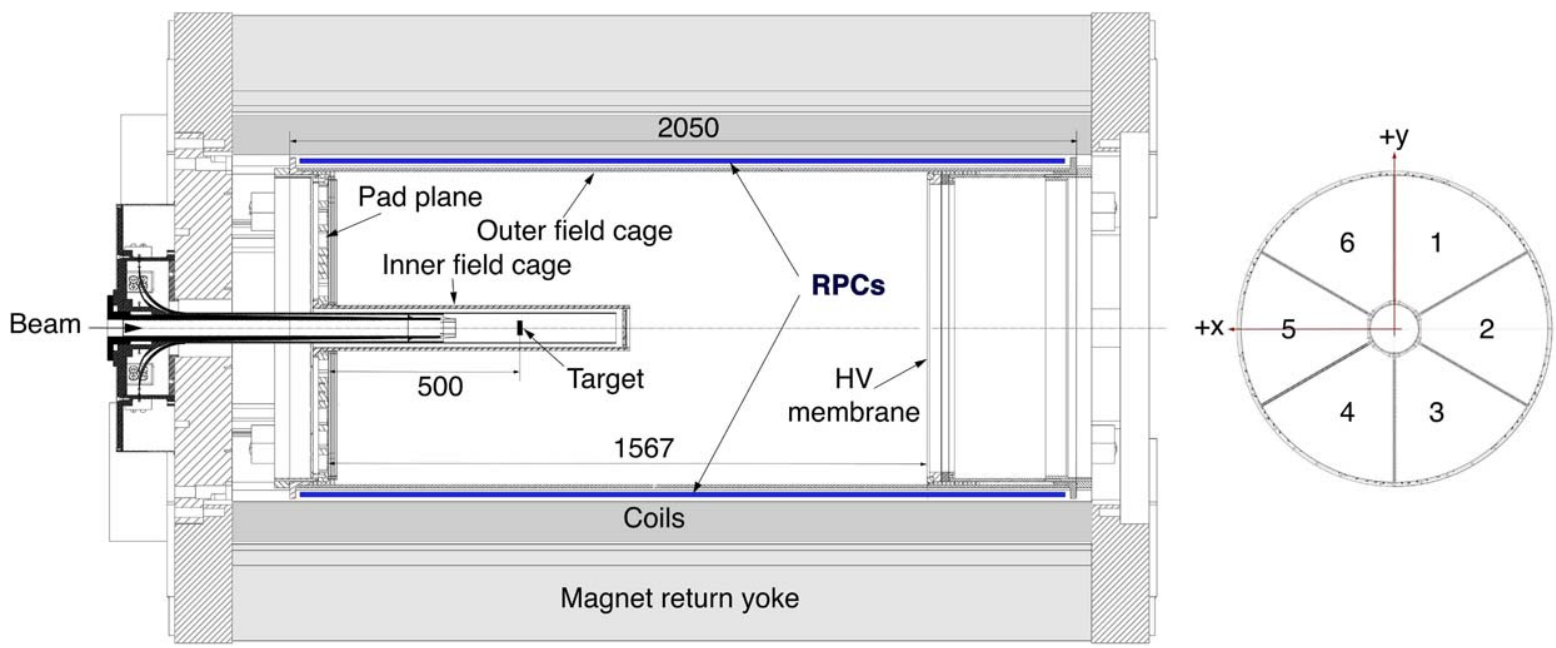

Fig. 3 Longitudinal cut through the TPC and the solenoidal magnet; the beam enters from the left side; the small figure to the right shows the layout of the six TPC readout sectors, looking downstream

of the beam optics and by multiple scattering. The nominal beam position ${ }^{3}$ was at $x_{\text {beam }}=y_{\text {beam }}=0$, however, excursions by several millimetres could occur. ${ }^{4}$ A loose fiducial cut $\sqrt{x_{\text {beam }}^{2}+y_{\text {beam }}^{2}}<12 \mathrm{~mm}$ ensured full beam acceptance. The muon and electron contaminations of the pion beam, stated above, refer to this acceptance cut.

We select 'good' beam particles by requiring the unambiguous reconstruction of the particle trajectory with good $\chi^{2}$. In addition we require that the particle type is unambiguously identified. We select 'good' accelerator spills by requiring minimal intensity and a 'smooth' variation of beam intensity across the $400 \mathrm{~ms}$ long spill. ${ }^{5}$

\section{The large-angle spectrometer}

In HARP's large-angle region, a cylindrical TPC [1] had been chosen as tracking detector. It was embedded in a solenoidal magnet that generated a magnetic field of $0.7 \mathrm{~T}$

\footnotetext{
${ }^{3} \mathrm{~A}$ right-handed Cartesian and/or spherical polar coordinate system is employed; the $z$ axis coincides with the beam line, with $+z$ pointing downstream; the coordinate origin is at the centre of the beryllium target, $500 \mathrm{~mm}$ downstream of the TPC's pad plane; looking downstream, the $+x$ coordinate points to the left and the $+y$ coordinate points up; the polar angle $\theta$ is the angle with respect to the $+z$ axis; when looking downstream, the azimuthal angle $\phi$ increases in the clockwise direction, with the $+x$ axis at $\phi=0$.

${ }^{4}$ The only relevant issue is that the trajectory of each individual beam particle is known, whether shifted or not, and therefore the amount of matter to be traversed by the secondary hadrons.

${ }^{5} \mathrm{~A}$ smooth variation of beam intensity eases corrections for dynamic TPC track distortions.
}

parallel to the TPC axis. The magnet was in general operated with its polarity tied to the beam polarity. ${ }^{6}$

The TPC filled most of the inner bore of the magnet, leaving a $25 \mathrm{~mm}$ wide gap between TPC and magnet coils. This gap was used to house two overlapping layers of $2 \mathrm{~m}$ long RPCs [2] directly mounted onto the outer field cage of the TPC.

The layout of the TPC and its position in the solenoidal magnet is shown in Fig. 3. The TPC has an external diameter of $832 \mathrm{~mm}$ and an overall length of $\sim 2 \mathrm{~m}$. It consists of two Stesalit cylinders forming the inner and outer field cages, a wire chamber with pad readout, located at the upstream end, and a high-voltage (HV) membrane at $1567 \mathrm{~mm}$ distance from the pad plane. The inner field cage extends over about half of the drift volume; it encloses the target, the centre of which is located $500 \mathrm{~mm}$ downstream of the pad plane.

The tracking volume extends radially from $75 \mathrm{~mm}$ to $385 \mathrm{~mm}$ and over $\sim 1.5 \mathrm{~m}$ longitudinally. Electrons from ionization induced by charged particles in the TPC gas drift upstream under the influence of the longitudinal electrical field; they are amplified in the wire chamber and read out through pads arranged in six identical sectors, as shown in Fig. 3. Each sector comprised 662 readout pads of dimensions $6.5 \times 15 \mathrm{~mm}^{2}$ arranged in 20 concentrical rows.

Our calibration work on the HARP TPC and RPCs is described in [1] and [2], and in references cited therein. In particular, we recall that static and dynamic TPC track distortions up to $\sim 10 \mathrm{~mm}$ have been corrected to better than $300 \mu \mathrm{m}$. Therefore, TPC track distortions do not affect the precision of our cross-section measurements.

${ }^{6}$ The HARP data-taking convention was that $B_{\mathrm{z}}>0$ refers to positive beam polarity. 


\section{Track reconstruction and particle identification}

\subsection{Pattern recognition in the TPC}

The clusters measured by the TPC constitute space points along the track trajectory. Each space point has three uniquely determined coordinates: $r, \phi$, and $z$. Our pattern recognition of tracks with $p_{\mathrm{T}} \geq 0.05 \mathrm{GeV} / c$ originating from the target region is based on the TOPAZ histogram technique [13]: a 2-dimensional histogram of the ratio $z / r$ against azimuthal angle $\phi$ is filled with all reconstructed clusters. Physical tracks populate one or two adjacent bins (the bin sizes are suitably chosen) and thus are easily recognised.

\subsection{Helix fit of TPC tracks}

For the fit of trajectories in the TPC we adopted the 'Generalized Least-Squares Fit' (GLSF) concept. This is the formal generalization of the standard least-squares fit for an arbitrary number of error-prone dimensions, and the solution of the equations resulting from the $\chi^{2}$ minimization with the Lagrange-multiplier method. The mathematical intricacies can be found in [14]. For the three parameters that describe the circle projection of a helix, we adopted the TOPAZ parametrization [15], for the attractive feature of avoiding any discontinuity in the numerical values of fit parameters. Most importantly, it features a smooth transition between the left and the right bend of high- $p_{\mathrm{T}}$ tracks in the solenoidal magnetic field, and hence avoids a discontinuity in the assignment of a positive or negative charge-signed $p_{\mathrm{T}}$. For more details on the parametrization and the fit procedure, we refer to [16].

The GLSF must start from reasonable starting values of the parameters that describe the helix. They are obtained by the Chernov-Ososkov algorithm [17].

Our GLSF algorithm yields the transverse momentum $p_{\mathrm{T}}$ of a track, its charge sign, its polar angle $\theta$, and its closest point of approach to the $z$ axis.

\subsection{Virtual beam point}

The $p_{\mathrm{T}}$ resolution of tracks can be improved by nearly a factor of two by the use of the beam point ${ }^{7}$ as an additional point to the trajectory in the TPC. The transverse coordinates of the beam point are known from the extrapolation of the trajectory of the incoming beam particle. Their errors stem from the extrapolation of the beam trajectory that is measured by MWPCs, and from multiple scattering of the beam particle. When the beam point is used in the fit, a third

\footnotetext{
${ }^{7}$ What we call 'beam point' is the best estimate of the interaction vertex of the incoming beam particle.
}

contribution to the error of its transverse coordinates arises from multiple scattering of the secondary particle in materials between the vertex and the TPC volume.

However, the correct error assignment to the beam point is not sufficient. Since a secondary track loses energy by ionization in the target and in materials between the vertex and the TPC volume, a correction must be calculated that replaces the real beam point by a 'virtual' beam point which is bias-free with respect to the extrapolation of the trajectory measured in the TPC. It is this virtual beam point, and not the real beam point, that is used in the (final) track fit. It is determined in an iterative procedure that starts from the fit of the track momentum in the TPC gas, including the real beam point. The fitted trajectory in the TPC gas is then backtracked to the beam particle trajectory taking the energy loss and multiple scattering into account. It renders a first estimate of the virtual beam point. Using this estimate, the track in the TPC gas is again fitted, and the procedure is iterated until the position of the virtual beam point is stable. Since in the calculation of the move from the real to the virtual beam point the energy loss is taken into account, and since the energy loss depends on the type of particle, three different virtual beam points are calculated according to the proton, pion, and electron hypotheses. Accordingly, three different track fits are performed.

The fit with the virtual beam point included gives the best possible estimate of the particle momentum in the TPC gas. In order to determine what is really needed, namely the momentum at the vertex, in a last step the particle is tracked back to the vertex, taking into account the energy loss under the three different particle hypotheses. The track parameters at the vertex are used for the determination of differential cross-sections.

\subsection{Particle identification algorithm}

The particles detected in HARP's large-angle region are protons, charged pions, and electrons ${ }^{8}$ (we disregard here small admixtures of kaons and deuterons which will be discussed in Sect. 7). The charged pion sample comprises muons from pion decay since the available instrumentation does not distinguish them from charged pions.

The $\mathrm{d} E / \mathrm{d} x$ and the time-of-flight methods of particle identification are considered independent.

To separate measured particles into species, we assign to each particle a probability of being a proton, a pion (muon), or an electron, respectively. The probabilities add up to unity, so that the number of particles is conserved.

Each track is characterized by four measured quantities: $p_{\mathrm{T}}$ (transverse momentum), $\theta$ (polar angle), $\beta$ (relative velocity) and $\mathrm{d} E / \mathrm{d} x$ (specific ionization). For particle identification purposes, these variables refer to reconstructed

\footnotetext{
${ }^{8}$ The term 'electron' also refers to positrons.
} 
('smeared') variables in both the data and the Monte Carlo simulation.

In every bin of $\left(p_{\mathrm{T}}, \theta\right)$, the probability $\mathcal{P}(i \mid \beta$, $\left.\mathrm{d} E / \mathrm{d} x, p_{\mathrm{T}}, \theta\right)$ of a particle to belong to species $i(i=1$ [proton], 2 [pion], 3 [electron]) in a mixture of protons, pions, and electrons is according to Bayes' theorem as follows:

$$
\begin{aligned}
& \mathcal{P}\left(i \mid \beta, \mathrm{d} E / \mathrm{d} x, p_{\mathrm{T}}, \theta\right) \\
& =\frac{P\left(\beta, \mathrm{d} E / \mathrm{d} x \mid i, p_{\mathrm{T}}, \theta\right) \cdot P\left(i, p_{\mathrm{T}}, \theta\right)}{\sum_{i=1}^{3}\left[P\left(\beta, \mathrm{d} E / \mathrm{d} x \mid i, p_{\mathrm{T}}, \theta\right) \cdot P\left(i, p_{\mathrm{T}}, \theta\right)\right]},
\end{aligned}
$$

where the sum

$$
\sum_{i=1}^{3} \mathcal{P}\left(i \mid \beta, \mathrm{d} E / \mathrm{d} x, p_{\mathrm{T}}, \theta\right)
$$

is normalized to unity. The probabilities $P\left(i, p_{\mathrm{T}}, \theta\right)$ are given by

$$
P\left(i, p_{\mathrm{T}}, \theta\right)=\frac{N_{i}\left(p_{\mathrm{T}}, \theta\right)}{\sum_{i=1}^{3} N_{i}\left(p_{\mathrm{T}}, \theta\right)},
$$

where $N_{i}\left(p_{\mathrm{T}}, \theta\right)$ is the number of particles of species $i$ in the respective data sample. Then (1) becomes

$$
\begin{aligned}
\mathcal{P}\left(i \mid \beta, \mathrm{d} E / \mathrm{d} x, p_{\mathrm{T}}, \theta\right) & \\
= & \frac{P\left(\beta, \mathrm{d} E / \mathrm{d} x \mid i, p_{\mathrm{T}}, \theta\right) \cdot N_{i}\left(p_{\mathrm{T}}, \theta\right)}{\sum_{i}^{3}\left[P\left(\beta, \mathrm{d} E / \mathrm{d} x \mid i, p_{\mathrm{T}}, \theta\right) \cdot N_{i}\left(p_{\mathrm{T}}, \theta\right)\right]} .
\end{aligned}
$$

We note that in (1) and (2) the term $P\left(\beta, \mathrm{d} E / \mathrm{d} x \mid i, p_{\mathrm{T}}, \theta\right)$ denotes a probability density function which is normalized to unity. This probability density function must represent the data in the bin $\left(p_{\mathrm{T}}, \theta\right)$.

Before determining the probability represented by (2), the probability density functions $P\left(\beta, \mathrm{d} E / \mathrm{d} x \mid i, p_{\mathrm{T}}, \theta\right)$ and the particle abundances $N_{i}\left(p_{\mathrm{T}}, \theta\right)$ must be known. This seemingly circular situation is resolved by an iterative comparison of data with the Monte Carlo simulation, to achieve agreement of the distributions in both variables $\beta$ and $\mathrm{d} E / \mathrm{d} x$. With a view to starting from abundances as realistic as possible, the comparison is initially limited to regions in phase space where the particle species are unambiguously separated from each other in either $\mathrm{d} E / \mathrm{d} x$ or $\beta$. In other words, the few parameters that govern the probability density functions and the particle abundances are determined from the data in every bin of $\left(p_{\mathrm{T}}, \theta\right)$.

In case one of the two identification variables is absent, ${ }^{9}$ only the other is used. In the rare cases where both identification variables are absent, the identification probabilities reproduce the estimated particle abundances.

${ }^{9}$ For example, because of too few clusters to calculate $\mathrm{d} E / \mathrm{d} x$, or a missing RPC pad.

\subsection{Particle abundances}

Particle abundances cannot a priori be expected to be correct in the Monte Carlo simulation. Therefore in general the particles must be weighted such that data and Monte Carlo distributions agree.

We had expected that the Monte Carlo simulation tool kit Geant4 [18, 19] would provide us with reasonably realistic spectra of secondary hadrons. We found this expectation more or less met by Geant4's so-called QGSP_BIC physics list, but only for the secondaries from incoming beam protons. For the secondaries from incoming beam pions, we found the standard physics lists of Geant4 unsuitable [20].

To overcome this problem, we built our own HARP_CDP physics list for the production of secondaries from incoming beam pions. It starts from Geant4's standard QBBC physics list, but the Quark-Gluon String Model is replaced by the FRITIOF string fragmentation model for kinetic energy $E>$ $6 \mathrm{GeV}$; for $E<6 \mathrm{GeV}$, the Bertini Cascade is used for pions, and the Binary Cascade for protons; elastic and quasi-elastic scattering is disabled. Examples of the good performance of the HARP_CDP physics list are given in [20].

Figure 4 demonstrates the level of overall agreement between data and Monte Carlo simulation in the variable $1 / p_{\mathrm{T}}$, after convergence of the iterative procedure to determine the smooth weighting functions to the latter. The figure also shows, for incoming protons and for a typical polar-angle range, the subdivision of the data into particle species by applying the particle identification weights.

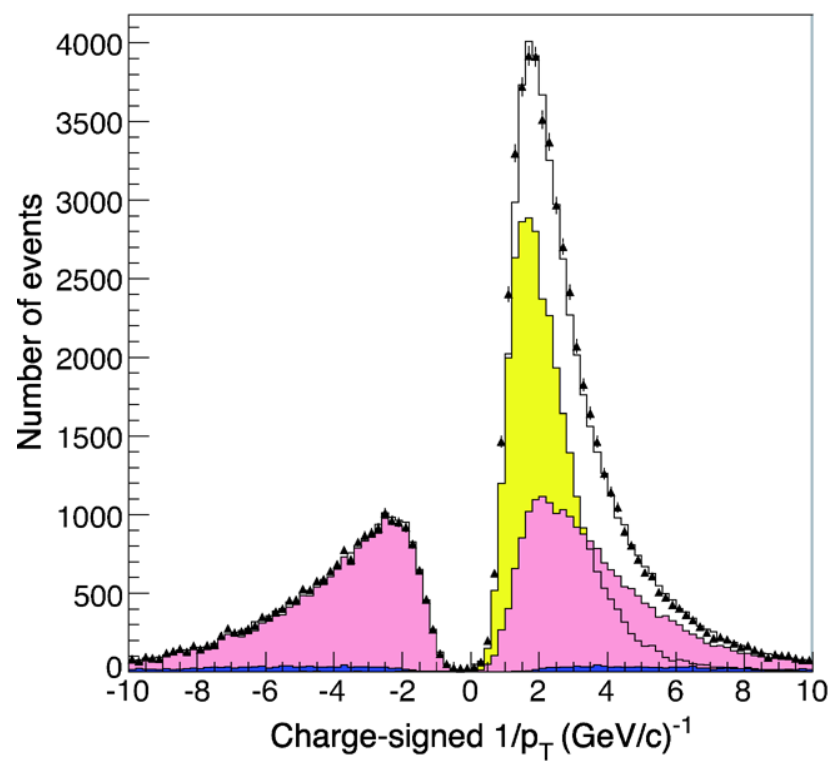

Fig. $41 / p_{\mathrm{T}}$ spectra of the secondary particles from $+8.9 \mathrm{GeV} / \mathrm{c}$ beam protons on a $5 \% \lambda_{\text {abs }}$ Be target, for polar angles $50^{\circ}<\theta<60^{\circ}$; black triangles denote data, the solid lines Monte Carlo simulation; the shaded histograms show the subdivision of the data into particle species by applying the particle identification weights: light shading denotes protons, medium shading pions, and dark shading electrons 
Once the abundances are determined, for any pair of $\mathrm{d} E / \mathrm{d} x$ and $\beta$, and using the experimental resolution functions, the probability can be derived that the particle is a proton, a pion, or an electron. This probability is consistently used for weighting when entering tracks into plots or tables.

\section{Physics performance}

\subsection{Physics performance of the TPC}

In [1] the absolute momentum scale has been shown to be correct to better than $2 \%$, both for positively and negatively charged particles.

Figure 5(a) shows the $1 / p_{\mathrm{T}}$ difference for positive particles with $0.6<\beta<0.75$ and $45^{\circ}<\theta<65^{\circ}$, between the measurement in the TPC and the determination from RPC time of flight with the proton-mass hypothesis. The selection cuts ensure a practically pure sample of protons (the background from pions and kaons is negligible as suggested by the very small contribution of negative particles selected with the same cuts that are shown as dots in Fig. 5(a)). A net TPC resolution of $\sigma\left(1 / p_{\mathrm{T}}\right)=0.20(\mathrm{GeV} / c)^{-1}$ is obtained by subtracting the contribution of $\sim 0.18(\mathrm{GeV} / c)^{-1}$ from the time-of-flight resolution and fluctuations from energy loss and multiple scattering in materials between the vertex and the TPC volume quadratically from the convoluted resolution of $0.27(\mathrm{GeV} / \mathrm{c})^{-1}$.
Figure 5(b) shows the net TPC resolution $\sigma\left(1 / p_{\mathrm{T}}\right)$ as a function of $\beta$. Figure 5(c) shows the same as a function of $\theta$. The agreement with the expectation from a Monte Carlo simulation is satisfactory. The resolution $\sigma\left(1 / p_{\mathrm{T}}\right)$ is typically $0.2(\mathrm{GeV} / \mathrm{c})^{-1}$ and worsens towards small $\beta$ and small $\theta$. This is because in both cases the position error of the virtual beam point increases owing to increased multiple scattering in materials before the protons enter the TPC.

Data from the elastic scattering of incoming pions or protons on protons at rest have the added feature of a kinematical constraint. The possibility to calculate from the fourmomentum of the incoming beam particle and the polar angle $\theta$ the momentum of the large-angle recoil proton, permits a valuable cross-check of the TPC's $p_{\mathrm{T}}$ resolution. Figure 6 shows the result from the elastic scattering of incoming $+3 \mathrm{GeV} / c$ protons and $\pi^{+}$'s in a liquid hydrogen target. Here, the $p_{\mathrm{T}}$ of the recoil proton has been determined in the following two ways: $1 / p_{\mathrm{T}}^{\text {meas }}$ is determined from the reconstructed track curvature in the TPC; $1 / p_{\mathrm{T}}^{\text {pred }}$ is predicted from the elastic scattering kinematics from the polar angle of the recoil proton which is little affected by TPC track distortions. Figure 6 demonstrates a resolution of $\sigma\left(1 / p_{\mathrm{T}}\right) \sim 0.19(\mathrm{GeV} / c)^{-1}$ after unfolding a contribution of $\sigma\left(1 / p_{\mathrm{T}}\right) \sim 0.12(\mathrm{GeV} / c)^{-1}$ from fluctuations from energy loss and multiple scattering in materials between the vertex and the TPC volume. The measured difference in $p_{\mathrm{T}}$ is $0.8 \%$, in line with the $2 \%$ uncertainty of the momentum scale.
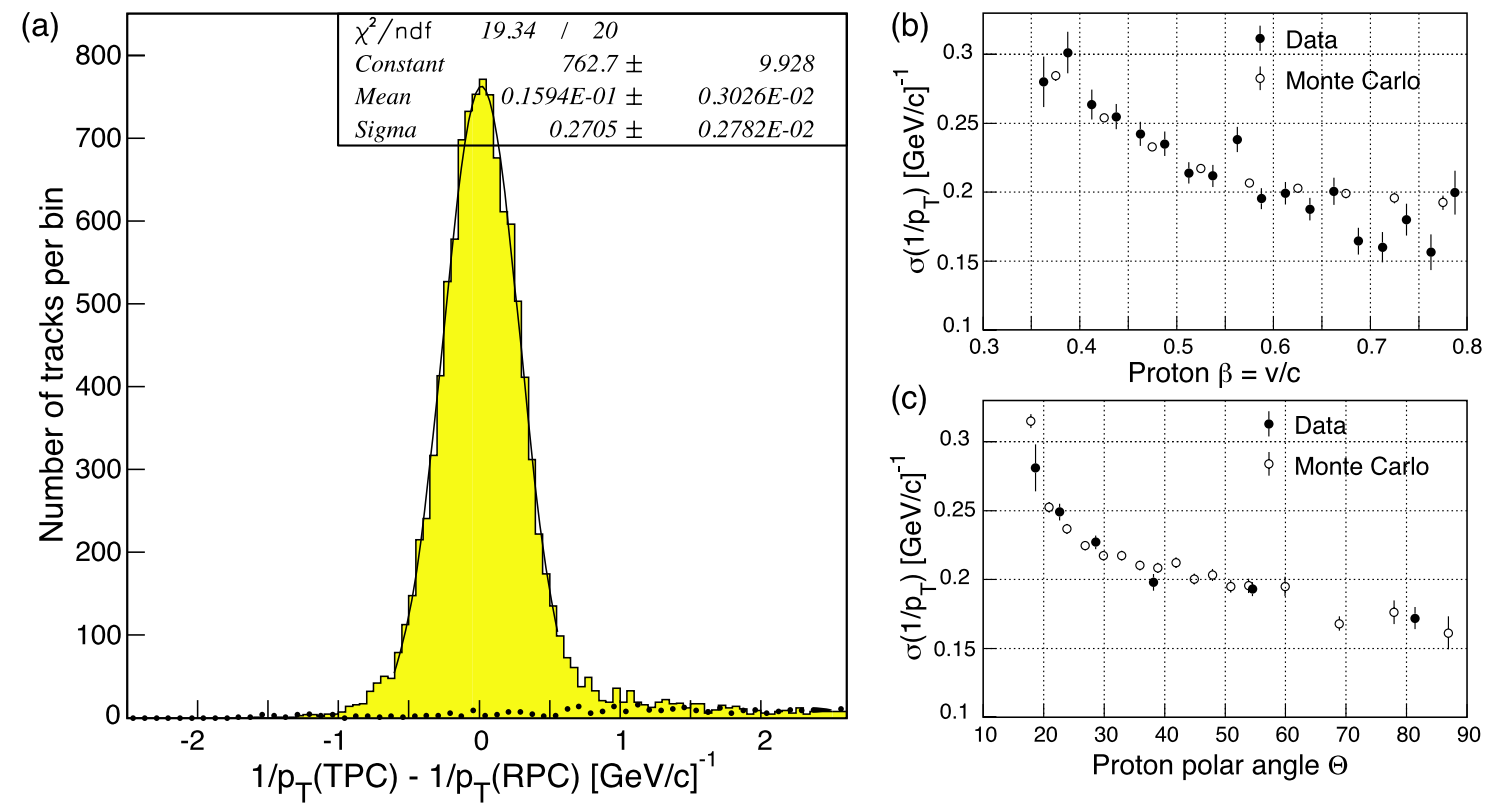

Fig. 5 (a) Difference of the inverse transverse momenta of positive (shaded histogram) and negative (dots) particles from the measurement in the TPC and from the determination from RPC time of flight, for $0.6<\beta<0.75$ and for $45^{\circ}<\theta<65^{\circ}$; the positive particles are pro- tons, with a very small background from pions and kaons; (b) $\sigma\left(1 / p_{\mathrm{T}}\right)$ of protons with $45^{\circ}<\theta<65^{\circ}$ as a function of their relative velocity $\beta$; (c) $\sigma\left(1 / p_{\mathrm{T}}\right)$ of protons with $0.6<\beta<0.75$ as a function of their polar angle $\theta$ 


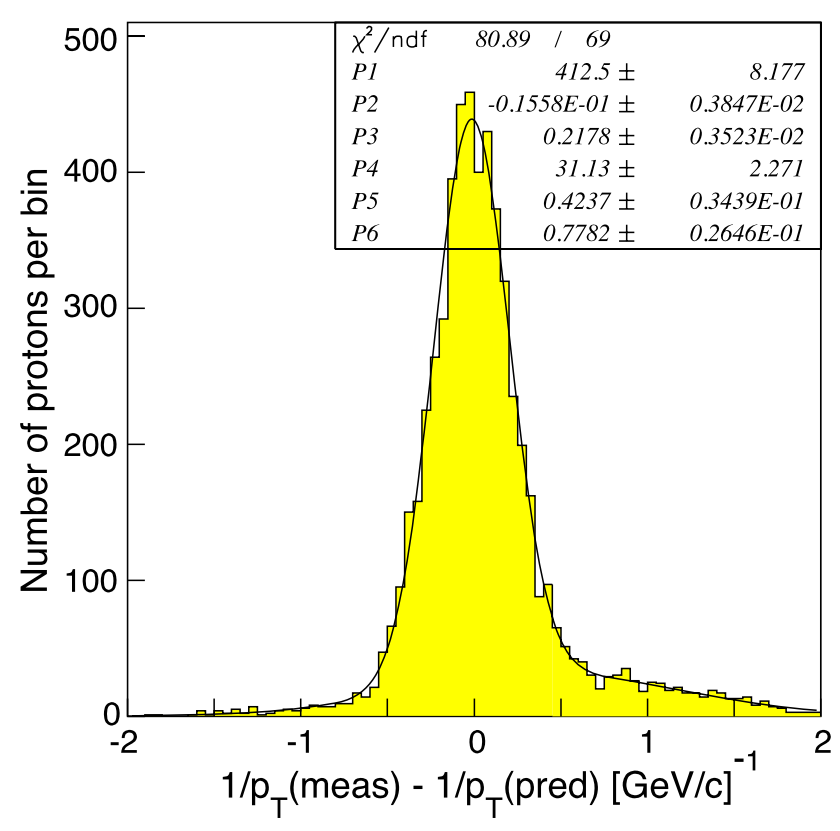

Fig. 6 The difference $1 / p_{\mathrm{T}}^{\text {meas }}-1 / p_{\mathrm{T}}^{\text {pred }}$ from large-angle recoil protons in elastic scattering events from a $+3 \mathrm{GeV} / c$ beam impinging on a liquid hydrogen target; the tail at the right side reflects the Landau tail in the proton energy loss in materials between the interaction point and the TPC volume

The polar angle $\theta$ is measured in the TPC with a resolution of $\sim 9 \mathrm{mrad}$, for a representative angle of $\theta=60^{\circ}$. To this a multiple scattering error has to be added which is $\sim 7 \mathrm{mrad}$ for a proton with $p_{\mathrm{T}}=500 \mathrm{MeV} / c$ and $\theta=60^{\circ}$, and $\sim 4 \mathrm{mrad}$ for a pion with the same characteristics. The polar-angle scale is correct to better than $2 \mathrm{mrad}$.

Besides the $p_{\mathrm{T}}$ and the polar angle $\theta$ of tracks, the TPC also measures $\mathrm{d} E / \mathrm{d} x$ with a view to particle identification. The $\mathrm{d} E / \mathrm{d} x$ resolution is $16 \%$ for a track length of $300 \mathrm{~mm}$.

\subsection{Physics performance of the RPCs}

The intrinsic efficiency of the RPCs that surround the TPC is better than $98 \%$. While the system efficiency for pions with $p_{\mathrm{T}}>100 \mathrm{MeV} / c$ at the vertex is close to the intrinsic efficiency, it is slightly worse for protons because of their higher energy loss in structural materials. Protons with $p<$ $350 \mathrm{MeV} / c$ at the vertex get absorbed before they reach the RPCs and thus escape time-of-flight measurement.

The intrinsic time resolution of the RPCs is $127 \mathrm{ps}$ and the system time-of-flight resolution (that includes the jitter of the arrival time of the beam particle at the target) is $175 \mathrm{ps}$.

Figure 7(a) shows the specific ionization $\mathrm{d} E / \mathrm{d} x$, measured by the TPC, and Fig. 7(b) the relative velocity $\beta$ from the RPC time of flight, of positive and negative secondaries, as a function of the momentum measured in the TPC. The figures demonstrate that in general protons and pions are
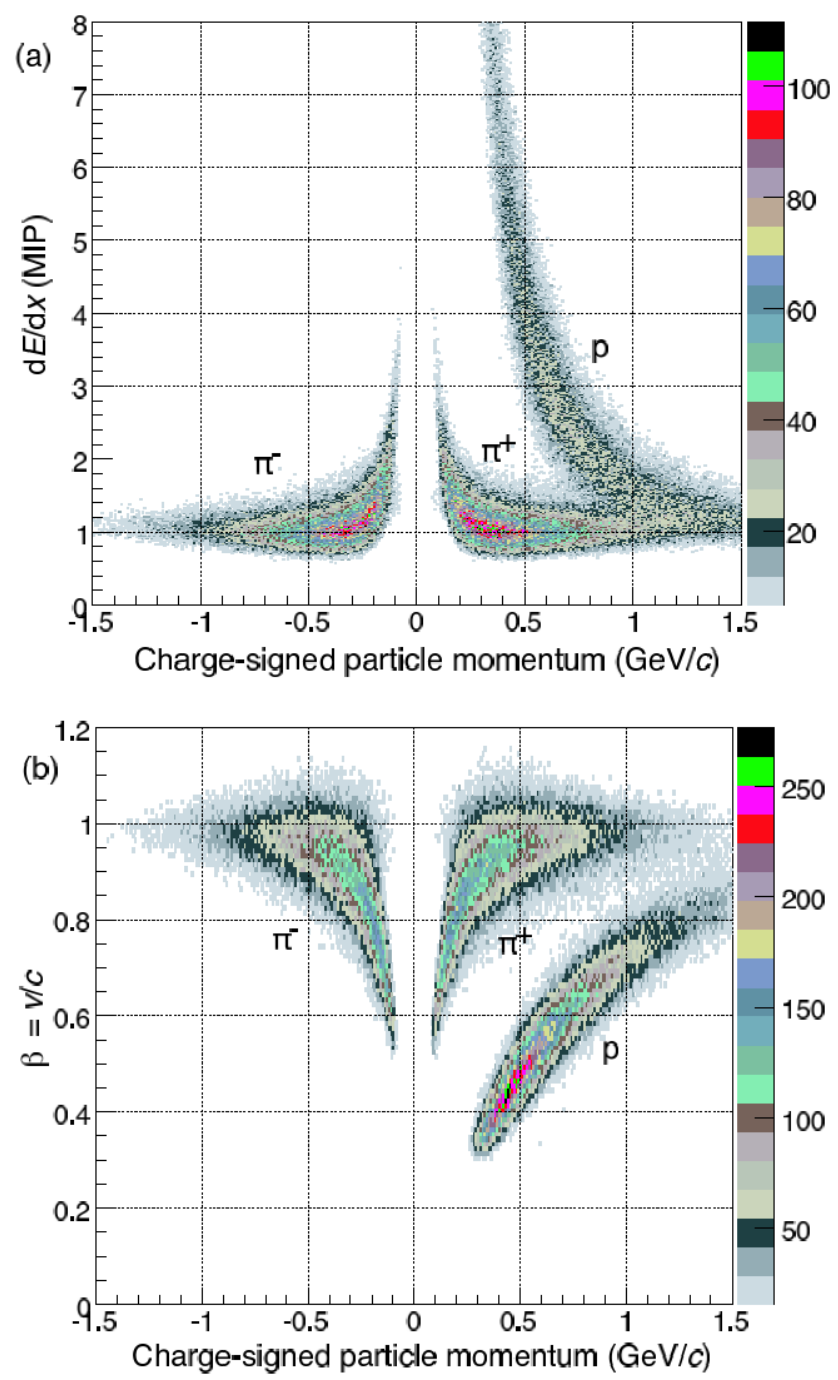

Fig. 7 (a) Specific ionization $\mathrm{d} E / \mathrm{d} x$ and (b) velocity $\beta$, versus the charge-signed momentum of positive and negative tracks in $+8.9 \mathrm{GeV} / c$ data; the boxes at the right side indicate the event statistics

well separated. They also underline the importance of the complementary separation by RPC time of flight at large particle momentum.

\section{Normalized secondary particle flux}

The measurement of the inclusive double-differential crosssection $\mathrm{d}^{2} \sigma / \mathrm{d} p \mathrm{~d} \Omega$ requires the flux of incoming beam particles, the number of target nuclei, and the number of secondary particles in bins of momentum $p$ and polar angle $\theta$. We shall discuss these elements in turn.

\subsection{Beam intensity}

The event trigger had two levels. A first, loose, level required only time-coincident signals from beam scintillation coun- 
ters. Irrespective of an interaction in the target, each 64th coincidence signal requested data readout as 'beam trigger'. A second, tighter level required in addition a signal in a cylindrical scintillator fibre detector that surrounded the target region, or a signal in a plane of scintillators in the forward direction (termed 'FTP' in Fig. 1). Each such 'event trigger' also requested data readout.

To achieve the wanted event statistics, the experiment was typically run with a dead time in excess of $50 \%$, given the $400 \mathrm{~ms}$ long accelerator spill and a readout time of order $1 \mathrm{~ms}$ per event. Since the dead time affects the beam trigger and the event trigger in the same way, it cancels in the cross-section calculation. For a given data set, the flux of incoming beam particles is defined by the number of beam triggers, multiplied by the scale-down factor of 64 . It is imperative, though, that the same cuts on the quality of the trajectory of the beam particle and on its identification be applied for accepted beam triggers and for accepted event triggers.

The efficiencies of both the beam trigger and the event trigger are very close to $100 \%$, thanks to majority requirements. The beam trigger efficiency cancels. For the event trigger, we determined an efficiency of $(99.0 \pm 0.2) \%$.

\subsection{Target}

The target was a cylinder made of high-purity (99.95\%) beryllium, with a density of $1.85 \mathrm{~g} / \mathrm{cm}^{3}$, a radius of $15 \mathrm{~mm}$, and a thickness of $20.5 \pm 0.1 \mathrm{~mm}\left(5 \% \lambda_{\text {abs }}\right)$.

The finite thickness of the target leads to a small attenuation of the number of incident beam particles. The attenuation factor is $f_{\text {att }}=0.975$.

\subsection{Track counting in bins of $p_{\mathrm{T}}$ and $\theta$}

This paper is concerned with determining inclusive crosssections of secondaries from the interactions of protons and pions with beryllium nuclei. This means that for a given data set, the secondaries are weighted with their probability of being a proton, a pion, or an electron, counted in bins of $p_{\mathrm{T}}$ and $\theta$, and related to the number of incoming beam particles and the number of target nuclei. The counting of secondaries is done in an integral way without regard to track-event relations.

Electrons stem primarily from the conversion of photons from $\pi^{0}$ decays. They tend to concentrate at small momenta. Below $150 \mathrm{MeV} / c$, they are identified by both $\mathrm{d} E / \mathrm{d} x$ and time of flight from the RPCs. From 150 to $250 \mathrm{MeV} / c$, the $\mathrm{d} E / \mathrm{d} x$ of pions and electrons coincides and they are only identified by time of flight. The Geant 4 electron abundance is compared with data in the region of good separation, as a function of momentum, and weighted to agree with the data. In the region of bad separation the electrons are subtracted using not the electron abundance predicted by Geant 4 , but the weighted prediction extrapolated from the region of good separation. Therefore, the Geant 4 prediction is used only through its extrapolated prediction of the energy dependence of electrons with momentum larger than $250 \mathrm{MeV} / c$.

Since the particle identification algorithm assigns to every particle a probability of being a proton, a pion, or an electron, the elimination of electrons from the samples of secondary protons and pions is straightforward.

It is justified to think of secondary tracks as originating exclusively from proton and pion interactions: interactions of beam electrons might occasionally lead to lowmomentum electron or positron tracks in the TPC, however, such tracks are recognized by the particle identification algorithm and disregarded in hadron production crosssections. Interactions of beam muons can be neglected.

Kaon and deuteron secondaries are initially part of pions and protons, respectively. Their identification is dealt with in Sect. 7.

\subsection{Track selection cuts}

We have a selection of 'good' TPC sectors: we discard tracks from the 'horizontal' sectors 2 and 5 out of the six sectors (see Fig. 3) for reasons of much worse than average performance, and the lack of reliable track distortion corrections [1].

Tracks are accepted if there are at least 10 TPC clusters along the trajectory.

A cut in the azimuthal angle $\phi$ is applied to avoid the dead regions of the six 'spokes' that subdivide the TPC pad plane into six sectors: $10^{\circ}$ on one side and $2^{\circ}$ on the other side of each spoke for tracks of one charge, and vice versa for the other charge. The asymmetric cut is motivated by the opposite bending of positive and negative tracks in the magnetic field.

The polar-angle range of tracks is limited to the range $20^{\circ}<\theta<125^{\circ}$. Tracks are also required to point back to the target, within the resolution limits.

\subsection{Correction for inefficiencies of track reconstruction and track selection}

The track reconstruction efficiency was determined by eyeball scanning of several thousand events by several physicists, with consistent results among them. The large number of scanned events permits us to determine the reconstruction efficiency as a function of geometric or kinematic variables. For example, Fig. 8(a) shows the reconstruction efficiency as a function of $1 / p_{\mathrm{T}}$ for all cases where the human eye finds at least five (out of a maximum of 20) clusters along a trajectory. The average reconstruction efficiency is between 

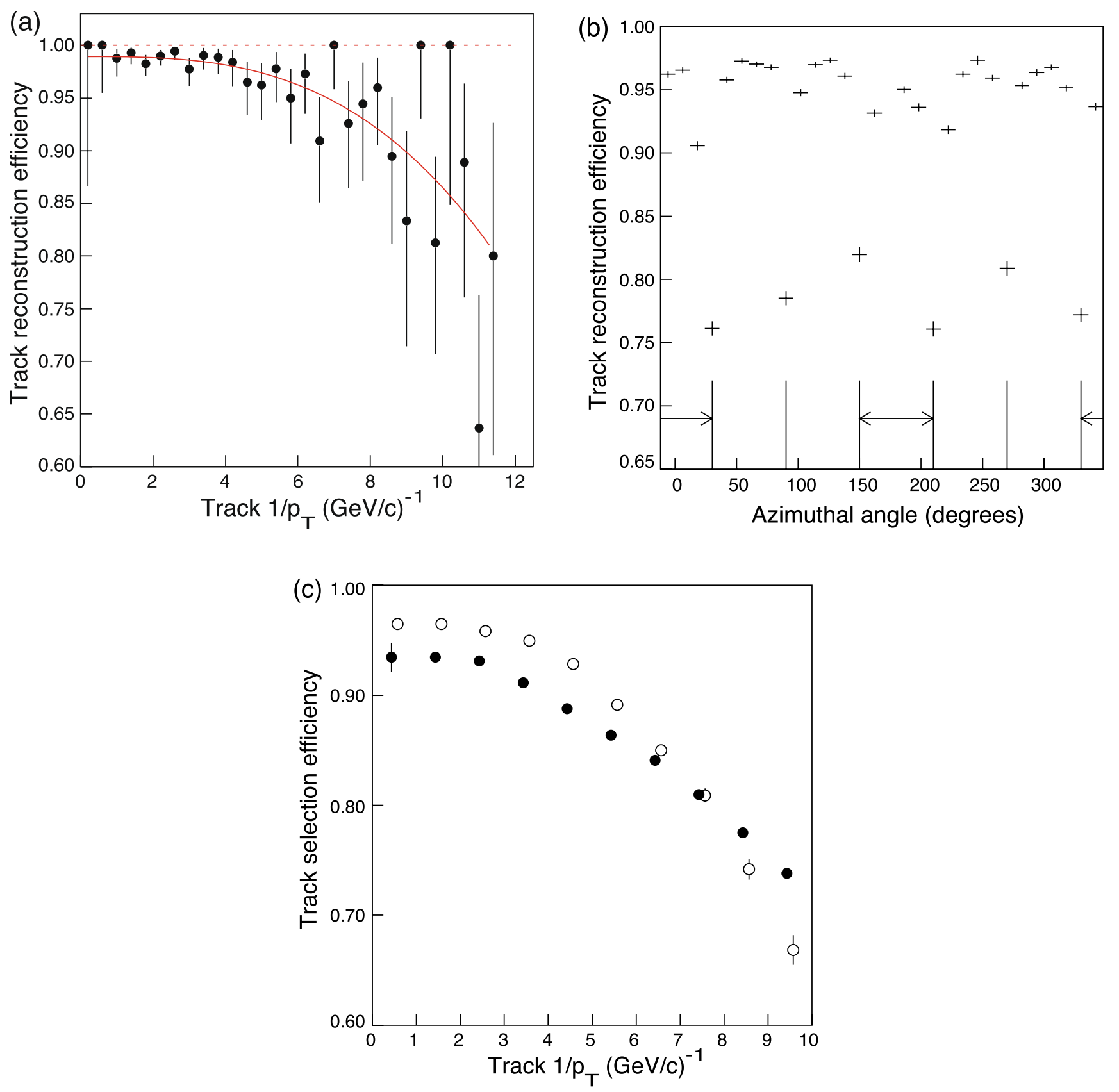

Fig. 8 (a) Track reconstruction efficiency from the eyeball scan; (b) track reconstruction efficiency from RPC hits; the vertical lines denote TPC sector boundaries where the TPC 'spokes' render the efficiency lower; the azimuthal ranges of the two TPC sectors not used for

$95 \%$ and $97 \%,{ }^{10}$ where the $2 \%$ range reflects the variation between different data sets.

We cross-checked the track reconstruction efficiency by requiring an RPC hit and at least two TPC clusters in the

\footnotetext{
${ }^{10}$ This average holds for tracks with $\left|p_{\mathrm{T}}\right|>0.1 \mathrm{GeV} / \mathrm{c}$ and $20^{\circ}<\theta<$ $125^{\circ}$, with safe distance from the insensitive azimuthal regions caused by the TPC 'spokes'.
}

the analysis are indicated by arrows; (c) efficiency of 10 or more TPC clusters for protons (open circles) and for pions (black circles); the errors shown are statistical only and mostly smaller than the symbol size

cone that is subtended by the respective RPC pad, as seen from the vertex. Figure 8(b) shows the resulting reconstruction efficiency as a function of the track's azimuthal angle. Outside the TPC spokes and within the four 'good' TPC sectors, the reconstruction efficiency determined that way agrees with the result from the eyeball scan.

The requirement of a minimum of 10 TPC clusters per track entails a loss that must be accounted for. Since the 
TPC cluster charge is in general larger for protons ${ }^{11}$ than for pions, the loss from this cut is different for protons and pions. Figure 8(c) shows the efficiency of requiring 10 or more TPC clusters, determined from the data, as a function of $1 / p_{\mathrm{T}}$, separately for protons and pions (the average number of clusters was $\sim 14$ ).

The overall track efficiency was taken as the product of the track reconstruction efficiency and the probability of having at least 10 clusters along the trajectory.

\subsection{Further corrections}

In this section, we discuss a few more corrections that are applied to the data. In general, they are determined from a Monte Carlo simulation that reproduces the migration of track parameters from generated ('true') to reconstructed ('smeared') ones. This concerns effects arising from finite resolution, charge misidentification, pion decays into muons, and re-interactions of secondaries in materials between the vertex and the TPC volume. ${ }^{12}$ There is also backscattering of particles from the solenoid coil at large radius back into the TPC, however, tracks from backscattering are eliminated by the requirement that they originate from the target.

Other than for the transverse momentum $p_{\mathrm{T}}$, migration is nearly negligible in the measurement of the polar angle $\theta$.

Charge misidentification occurs only at large transverse momentum, at the level of a few per cent. For example, a few 'antiprotons' at large transverse momentum are chargemisidentified protons and treated accordingly in the migration correction.

Pion decay into muons occurs at the typical level of $2 \%$. When the pion decay occurs in the first few centimetres of the flight path, the phenomenon is taken care of by the migration correction. When the pion decay occurs later, the track is likely to be lost because of the requirement that it originates from the target. Therefore, each pion receives a weight that compensates on the average for the loss from decay along a path of $200 \mathrm{~mm}$ length.

The re-interaction of secondaries takes place in the target material or in other materials between the target and the TPC volume. The typical probability for re-interaction is $3 \%$ for the former, and $2 \%$ for the latter. The re-interaction leads to tracks with other parameters than the initial track, and is taken care of by the migration correction.

\footnotetext{
${ }^{11}$ The lower proton velocity leads to higher specific ionization.

${ }^{12}$ These re-interactions, especially in the target material, are different from re-interactions of secondaries in the nuclear matter of the same nucleus in which the incoming beam particle interacted; the latter is an integral part of the inclusive cross-section reported in this paper.
}

6.7 Systematic errors

The systematic precision of our inclusive cross-sections is at the few-per-cent level, from errors in the normalization, in the momentum measurement, in particle identification, and in the corrections applied to the data.

The systematic error of the absolute flux normalization is taken as $2 \%$. This error arises from uncertainties in the target thickness, in the contribution of large-angle scattering of beam particles, in the attenuation of beam particles in the target, and in the subtraction of the muon and electron contaminations. Another contribution comes from the removal of events with an abnormally large number of TPC hits above threshold. ${ }^{13}$

The systematic error of the track finding efficiency is taken as $1 \%$ which reflects differences between results from different persons who conducted eyeball scans. We also take the statistical errors of the parameters of a fit to scan results as shown in Fig. 8(a) as systematic error into account. The systematic error of the correction for losses from the requirement of at least 10 TPC clusters per track is taken as $20 \%$ of the correction which itself is in the range of 5 to $30 \%$. This estimate arose from differences between the four TPC sectors that were used in our analysis, and from the observed variations with time.

The systematic error of the $p_{\mathrm{T}}$ scale is taken as $2 \%$ as discussed in [1].

The systematic errors of the proton, pion, and electron abundances are taken as $10 \%$. We stress that errors on abundances only lead to cross-section errors in case of a strong overlap of the resolution functions of both identification variables, $\mathrm{d} E / \mathrm{d} x$ and $\beta$. The systematic error of the correction for migration, absorption of secondary protons and pions in materials, and for pion decay into muons, is taken as $20 \%$ of the correction, or $1 \%$ of the cross-section, whichever is larger. These estimates reflect our experience with remanent differences between data and Monte Carlo simulations after weighting Monte Carlo events with smooth functions with a view to reproducing the data simultaneously in several variables in the best possible way.

All systematic errors are propagated into the momentum spectra of secondaries and then added in quadrature.

\section{Kaon and deuteron production}

The statistics from the $+8.9 \mathrm{GeV} / \mathrm{c}$ beam on a $5 \% \lambda_{\text {abs }}$ beryllium target is much larger than for any other combination of beam and target. This permits us to investigate in

\footnotetext{
${ }^{13}$ Very rarely, because of apparatus malfunction, the number of TPC hits was much larger than possible for a physics event. Such events are considered unphysical and eliminated.
} 
this particular data set the production of $\mathrm{K}^{+}$'s and deuterons in addition to the dominant protons, $\pi^{+}$'s, and $\pi^{-}$'s. With a view to benefiting from the cancellation of systematic errors, we present results in terms of the ratios $\mathrm{K}^{+} / \pi^{+}$and $\mathrm{d} / \mathrm{p}$.

\subsection{Kaons}

Figure 9 shows the relative velocity $\beta$ of positive secondaries for the polar-angle range $20.5^{\circ}<\theta<25.3^{\circ}$ and momentum between 520 and $560 \mathrm{MeV} / c$. A logarithmic scale is employed to make $\mathrm{K}^{+}$production visible which is at the level of a few per cent of the $\pi^{+}$production. The $\mathrm{K}^{+}$signal shows up between the proton and $\pi^{+}$signal thanks to the good resolution of the $\beta$ measurement by the RPCs.

The $\mathrm{K}^{+}$signal is fitted with a Gaussian. The $\pi^{+}$signal is represented by a Gaussian together with a tail that is experimentally determined from the $\beta$ distribution of the $\pi^{-}$'s. The latter is shown with crosses in Fig. 9. A possible $\mathrm{K}^{-}$ contribution is minimized by a $\mathrm{d} E / \mathrm{d} x$ cut.

In order to maximize the time of flight and hence the separation power, we restrict the analysis to the forward region in the range $20^{\circ}<\theta<32^{\circ}$. The momentum is required to be in the range $400<p<700 \mathrm{MeV} / c$, and $\mathrm{d} E / \mathrm{d} x$ must be between $70 \%$ and $155 \%$ of the nominal value.

Several corrections must be made to the fit results of the relative $\mathrm{K}^{+}$abundance. Correcting for cuts on the charge of the RPC signal, made with a view to optimizing time-offlight resolution, reduces the signal by $5 \%$. The correction for the non-Gaussian tail of the $\beta$ distribution of $\mathrm{K}^{+}$'s in-

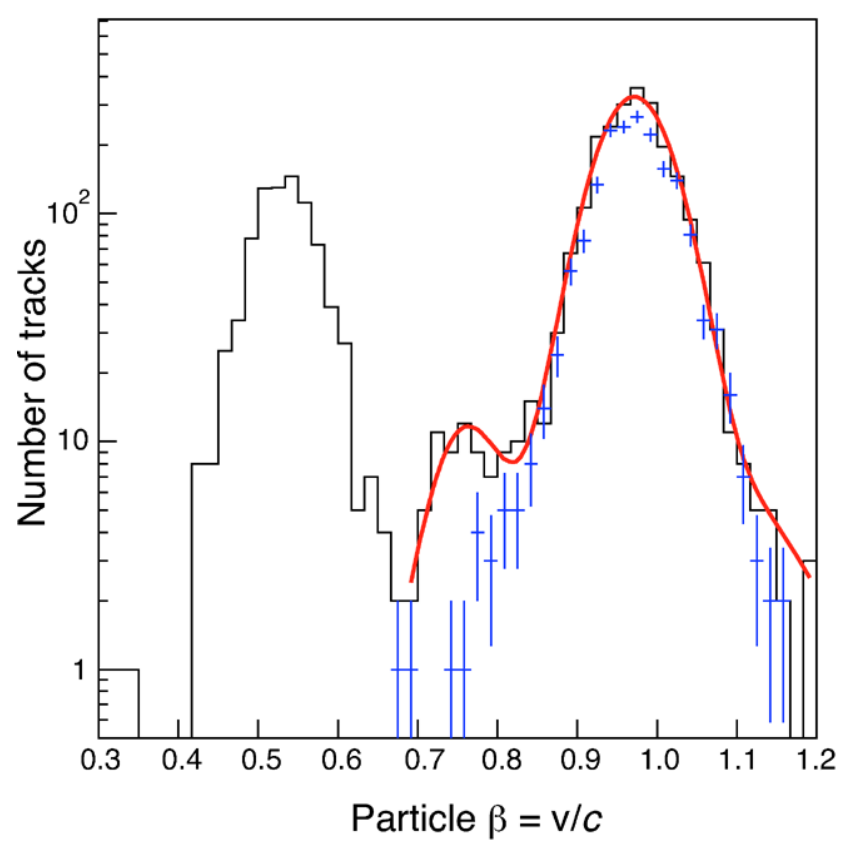

Fig. 9 Distribution of $\beta$ of positive secondaries on a logarithmic scale, with the $\mathrm{K}^{+}$signal showing up between pions and protons; the crosses show the $\beta$ distribution of $\pi^{-}$'s; the shown fit is explained in the text creases the signal by $8 \%$. The correction for different absorption of $\mathrm{K}^{+}$'s and $\pi^{+}$'s in structural materials increases the signal by $1 \%$.

Altogether, the resulting ratio is

$R_{\mathrm{K}}(p, \theta)=\frac{\mathrm{d}^{2} \sigma_{\mathrm{K}}(p, \theta) / \mathrm{d} p \mathrm{~d} \Omega}{\mathrm{d}^{2} \sigma_{\pi}(p, \theta) / \mathrm{d} p \mathrm{~d} \Omega}=0.020 \pm 0.003$

averaged over the said range of momentum and polar angle, and over the proton and $\pi^{+}$beams (in kaon production, no significant difference is seen between these beams). Figure 10 shows the $\mathrm{K}^{+} / \pi^{+}$ratio as a function of particle momentum and compares the measured ratios with the ratios from the FRITIOF and Binary Cascade hadron production models in Geant4. The data points are closer to the prediction by the FRITIOF model, however, the dependence on momentum is not reproduced. The agreement with the $\mathrm{Bi}$ nary Cascade model is poor.

\subsection{Deuterons}

Figure 11 shows the $\mathrm{d} E / \mathrm{d} x$ of positive secondaries for the polar-angle range $30^{\circ}<\theta<45^{\circ}$ and the momentum range from 500 to $600 \mathrm{MeV} / c$ (this momentum range refers to the momentum measured in the TPC and not to the momentum at the vertex). Pions and electrons are reduced by a loose time-of-flight cut. A clear signal of deuterons is visible at large $\mathrm{d} E / \mathrm{d} x$, next to the abundant protons.

In order to transform the ratio measured in the TPC volume to that at the vertex, appropriate corrections for the dif-

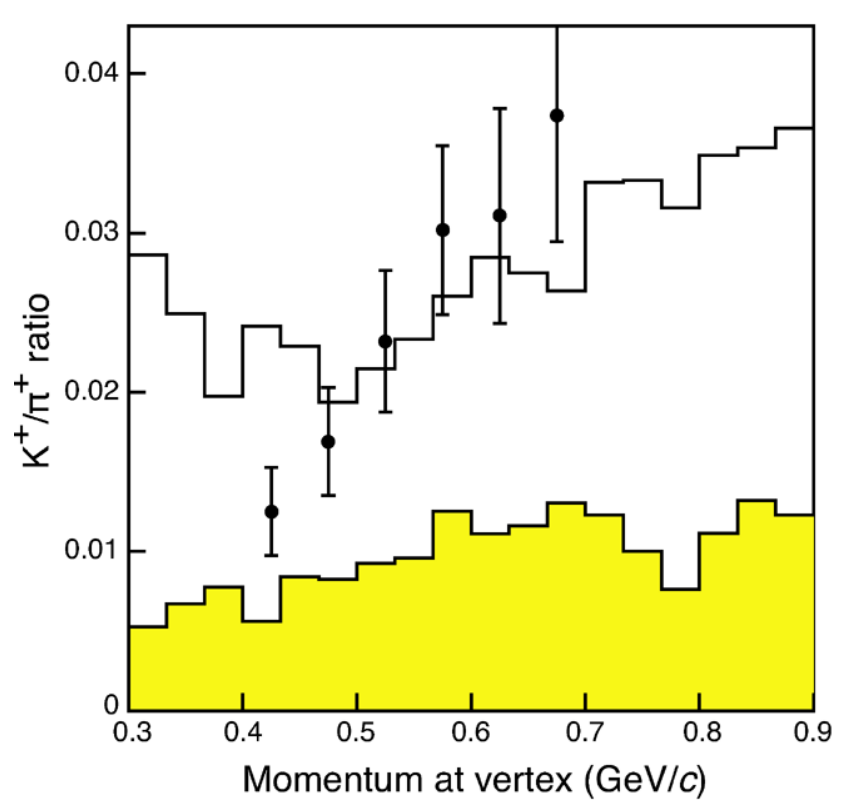

Fig. 10 Ratio of $\mathrm{K}^{+} / \pi^{+}$in $+8.9 \mathrm{GeV} / c$ proton and $\pi^{+}$interactions with beryllium nuclei; the data points (black circles) are closer to the prediction by the FRITIOF model (open histogram) in Geant4, but agree poorly with its Binary Cascade model (shaded histogram) 
Table 1 Ratio $R_{\mathrm{d}}$ of deuterons to protons, for different beam particles, averaged over the momentum at the vertex between $600 \mathrm{MeV} / c$ and $1050 \mathrm{MeV} / c$

\begin{tabular}{lccc}
\hline & $+8.9 \mathrm{GeV} / c$ protons & $+8.9 \mathrm{GeV} / c \pi^{+}$ & $-8.0 \mathrm{GeV} / c \pi^{-}$ \\
\hline $20^{\circ}<\theta<30^{\circ}$ & $0.051 \pm 0.004$ & $0.038 \pm 0.004$ & $0.05 \pm 0.01$ \\
$30^{\circ}<\theta<45^{\circ}$ & $0.076 \pm 0.004$ & $0.065 \pm 0.005$ & $0.07 \pm 0.01$ \\
$45^{\circ}<\theta<65^{\circ}$ & $0.113 \pm 0.009$ & $0.094 \pm 0.008$ & $0.13 \pm 0.02$ \\
$65^{\circ}<\theta<90^{\circ}$ & $0.17 \pm 0.03$ & $0.15 \pm 0.03$ & $0.16 \pm 0.03$ \\
$90^{\circ}<\theta<125^{\circ}$ & $0.26 \pm 0.04$ & $0.21 \pm 0.05$ & $0.30 \pm 0.05$ \\
\hline
\end{tabular}

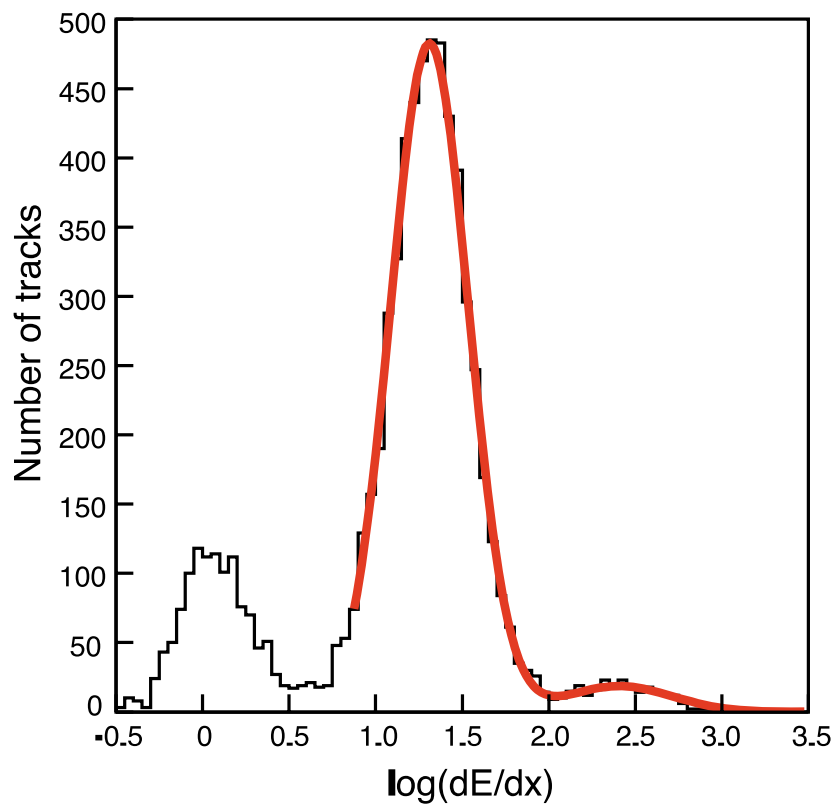

Fig. 11 Distribution of $\mathrm{d} E / \mathrm{d} x$ of positive secondaries, with the deuteron signal showing up at large $\mathrm{d} E / \mathrm{d} x$; see the text for the cuts applied; both the proton and deuteron signals are fitted with a Gaussian

ferent energy loss of protons and deuterons in materials between the vertex and the TPC volume, and for differences in the momentum spectra of protons and deuterons, must be applied. The results for the ratio

$R_{\mathrm{d}}(p, \theta)=\frac{\mathrm{d}^{2} \sigma_{\mathrm{d}}(p, \theta) / \mathrm{d} p \mathrm{~d} \Omega}{\mathrm{d}^{2} \sigma_{\mathrm{p}}(p, \theta) / \mathrm{d} p \mathrm{~d} \Omega}$

averaged over the momentum at the vertex between $600 \mathrm{MeV} / c$ and $1050 \mathrm{MeV} / c$, are given in Table 1.

The ratios $R_{\mathrm{d}}$ for the $+8.9 \mathrm{GeV} / c$ proton and $\pi^{+}$beams are shown in Fig. 12 for the polar-angle range $30^{\circ}<\theta<45^{\circ}$ as a function of the momentum at the vertex. We note that the deuteron abundance is reasonably well reproduced by the FRITIOF String Fragmentation model used in the Geant4 simulation tool kit, while it is underestimated by about one order of magnitude by the Binary Cascade model.

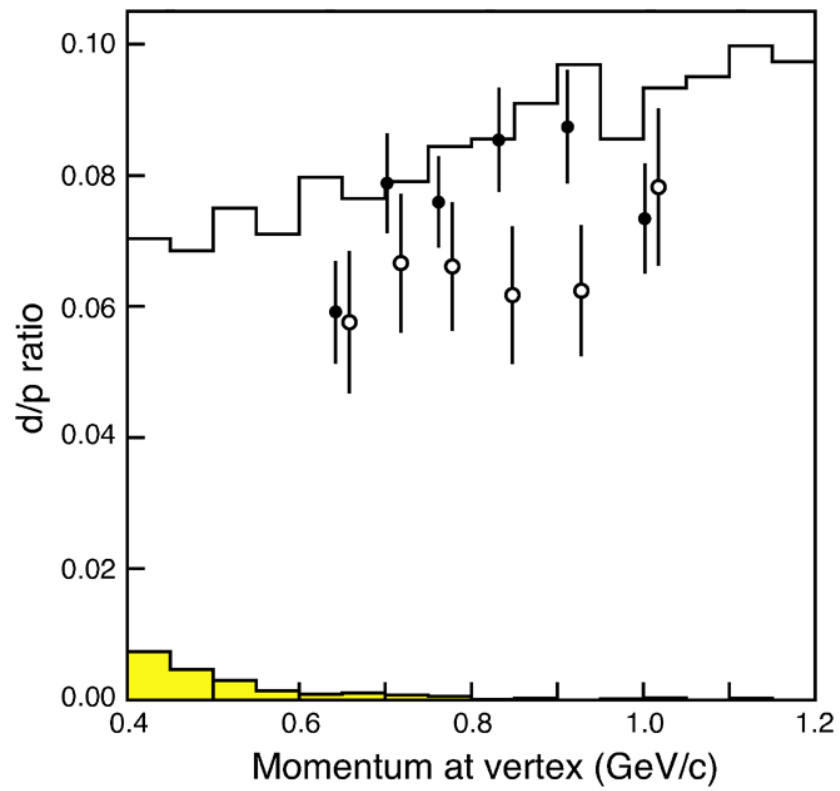

Fig. 12 Ratio $R_{\mathrm{d}}$ in $+8.9 \mathrm{GeV} / c$ proton and $\pi^{+}$interactions with beryllium nuclei, for $30^{\circ}<\theta<45^{\circ}$, as a function of the momentum at the vertex; black data points refer to the proton beam, open circles to the $\pi^{+}$beam. The proton beam data are compared with the predictions of the FRITIOF (open histogram) and the Binary Cascade (shaded histogram) models in Geant4

\section{Double-differential inclusive cross-sections of protons and pions}

In Tables $2-10$ we give the double-differential inclusive cross-sections $\mathrm{d}^{2} \sigma / \mathrm{d} p \mathrm{~d} \Omega$ for all nine combinations of incoming beam particle and secondary particle, including statistical and systematic errors. In each bin, the average momentum and the average polar angle are also given.

Cross-sections are only given if the total error is not larger than the cross-section itself. Since our track reconstruction algorithm is optimized for tracks with $p_{\mathrm{T}}$ above $\sim 70 \mathrm{MeV} / c$ in the TPC volume, we do not give crosssections from tracks with $p_{\mathrm{T}}$ below this value. Because of the absorption of slow protons in the material between the vertex and the TPC gas, and with a view to keeping the correction for absorption losses below 30\%, cross-sections from protons are limited to $p>350 \mathrm{MeV} / c$ at the interaction vertex. Proton cross-sections are also not given if a $10 \%$ 
Table 2 Double-differential inclusive cross-section $\mathrm{d}^{2} \sigma / \mathrm{d} p \mathrm{~d} \Omega[\mathrm{mb} /(\mathrm{GeV} / c \mathrm{sr})]$ of the production of protons in $\mathrm{p}+\mathrm{Be} \rightarrow \mathrm{p}+\mathrm{X}$ interactions with $+8.9 \mathrm{GeV} / c$ beam momentum; the first error is statistical, the second systematic; $p_{\mathrm{T}}$ in $\mathrm{GeV} / c$, polar angle $\theta$ in degrees

\begin{tabular}{|c|c|c|c|c|c|c|}
\hline \multirow[t]{2}{*}{$p_{\mathrm{T}}$} & \multicolumn{3}{|c|}{$20<\theta<30$} & \multicolumn{3}{|c|}{$30<\theta<40$} \\
\hline & $\left\langle p_{\mathrm{T}}\right\rangle$ & $\langle\theta\rangle$ & $\mathrm{d}^{2} \sigma / \mathrm{d} p \mathrm{~d} \Omega$ & $\left\langle p_{\mathrm{T}}\right\rangle$ & $\langle\theta\rangle$ & $\mathrm{d}^{2} \sigma / \mathrm{d} p \mathrm{~d} \Omega$ \\
\hline $0.20-0.24$ & 0.220 & 24.9 & $48.38 \pm 0.72 \pm 2.25$ & & & \\
\hline $0.24-0.30$ & 0.270 & 24.9 & $48.68 \pm 0.59 \pm 2.02$ & 0.271 & 35.0 & $42.76 \pm 0.54 \pm 1.65$ \\
\hline $0.30-0.36$ & 0.330 & 25.0 & $44.77 \pm 0.55 \pm 1.66$ & 0.329 & 35.0 & $41.00 \pm 0.52 \pm 1.39$ \\
\hline $0.36-0.42$ & 0.389 & 24.9 & $41.06 \pm 0.53 \pm 1.41$ & 0.389 & 35.0 & $38.10 \pm 0.51 \pm 1.20$ \\
\hline $0.42-0.50$ & 0.459 & 24.9 & $39.55 \pm 0.45 \pm 1.26$ & 0.459 & 34.9 & $33.39 \pm 0.42 \pm 1.00$ \\
\hline $0.50-0.60$ & 0.548 & 24.9 & $34.14 \pm 0.37 \pm 1.11$ & 0.548 & 34.9 & $27.89 \pm 0.34 \pm 0.87$ \\
\hline $0.60-0.72$ & 0.657 & 24.9 & $27.82 \pm 0.31 \pm 1.05$ & 0.656 & 34.9 & $21.47 \pm 0.27 \pm 0.80$ \\
\hline $0.72-0.90$ & & & & 0.801 & 34.8 & $14.52 \pm 0.19 \pm 0.74$ \\
\hline \multirow[t]{2}{*}{$p_{\mathrm{T}}$} & \multicolumn{3}{|c|}{$40<\theta<50$} & \multicolumn{3}{|c|}{$50<\theta<60$} \\
\hline & $\left\langle p_{\mathrm{T}}\right\rangle$ & $\langle\theta\rangle$ & $\mathrm{d}^{2} \sigma / \mathrm{d} p \mathrm{~d} \Omega$ & $\left\langle p_{\mathrm{T}}\right\rangle$ & $\langle\theta\rangle$ & $\mathrm{d}^{2} \sigma / \mathrm{d} p \mathrm{~d} \Omega$ \\
\hline $0.30-0.36$ & 0.330 & 45.0 & $38.81 \pm 0.50 \pm 1.15$ & & & \\
\hline $0.36-0.42$ & 0.389 & 45.0 & $36.20 \pm 0.49 \pm 0.99$ & 0.390 & 55.0 & $33.80 \pm 0.47 \pm 0.88$ \\
\hline $0.42-0.50$ & 0.458 & 44.9 & $30.11 \pm 0.40 \pm 0.86$ & 0.458 & 54.9 & $28.77 \pm 0.38 \pm 0.78$ \\
\hline $0.50-0.60$ & 0.548 & 44.9 & $23.87 \pm 0.32 \pm 0.78$ & 0.548 & 55.0 & $21.07 \pm 0.30 \pm 0.74$ \\
\hline $0.60-0.72$ & 0.656 & 44.9 & $17.87 \pm 0.25 \pm 0.70$ & 0.655 & 54.9 & $15.71 \pm 0.25 \pm 0.69$ \\
\hline $0.72-0.90$ & 0.801 & 44.8 & $11.38 \pm 0.17 \pm 0.61$ & 0.798 & 54.8 & $8.62 \pm 0.15 \pm 0.51$ \\
\hline $0.90-1.25$ & 1.035 & 44.7 & $3.96 \pm 0.07 \pm 0.35$ & 1.033 & 54.7 & $2.39 \pm 0.06 \pm 0.25$ \\
\hline \multirow[t]{2}{*}{$p_{\mathrm{T}}$} & \multicolumn{3}{|c|}{$60<\theta<75$} & \multicolumn{3}{|c|}{$75<\theta<90$} \\
\hline & $\left\langle p_{\mathrm{T}}\right\rangle$ & $\langle\theta\rangle$ & $\mathrm{d}^{2} \sigma / \mathrm{d} p \mathrm{~d} \Omega$ & $\left\langle p_{\mathrm{T}}\right\rangle$ & $\langle\theta\rangle$ & $\mathrm{d}^{2} \sigma / \mathrm{d} p \mathrm{~d} \Omega$ \\
\hline $0.36-0.42$ & 0.389 & 67.5 & $29.99 \pm 0.35 \pm 0.79$ & & & \\
\hline $0.42-0.50$ & 0.459 & 67.4 & $26.77 \pm 0.29 \pm 0.70$ & 0.458 & 81.9 & $19.01 \pm 0.25 \pm 0.63$ \\
\hline $0.50-0.60$ & 0.547 & 67.3 & $19.65 \pm 0.23 \pm 0.70$ & 0.546 & 81.6 & $12.23 \pm 0.18 \pm 0.54$ \\
\hline $0.60-0.72$ & 0.654 & 67.0 & $11.87 \pm 0.17 \pm 0.62$ & 0.653 & 81.5 & $6.04 \pm 0.13 \pm 0.41$ \\
\hline $0.72-0.90$ & 0.795 & 66.7 & $5.58 \pm 0.10 \pm 0.42$ & 0.795 & 81.3 & $2.42 \pm 0.07 \pm 0.24$ \\
\hline $0.90-1.25$ & 1.024 & 66.4 & $1.34 \pm 0.04 \pm 0.18$ & 1.026 & 81.5 & $0.56 \pm 0.02 \pm 0.09$ \\
\hline \multirow[t]{2}{*}{$p_{\mathrm{T}}$} & \multicolumn{3}{|c|}{$90<\theta<105$} & \multicolumn{3}{|c|}{$105<\theta<125$} \\
\hline & $\left\langle p_{\mathrm{T}}\right\rangle$ & $\langle\theta\rangle$ & $\mathrm{d}^{2} \sigma / \mathrm{d} p \mathrm{~d} \Omega$ & $\overline{\left\langle p_{\mathrm{T}}\right\rangle}$ & $\langle\theta\rangle$ & $\mathrm{d}^{2} \sigma / \mathrm{d} p \mathrm{~d} \Omega$ \\
\hline $0.36-0.42$ & & & & 0.388 & 113.3 & $7.21 \pm 0.15 \pm 0.27$ \\
\hline $0.42-0.50$ & 0.457 & 96.8 & $10.65 \pm 0.18 \pm 0.52$ & 0.456 & 113.2 & $4.82 \pm 0.11 \pm 0.23$ \\
\hline $0.50-0.60$ & 0.545 & 96.5 & $6.21 \pm 0.13 \pm 0.37$ & 0.543 & 112.7 & $2.28 \pm 0.07 \pm 0.18$ \\
\hline $0.60-0.72$ & 0.652 & 96.3 & $2.57 \pm 0.08 \pm 0.22$ & 0.650 & 112.0 & $0.75 \pm 0.04 \pm 0.09$ \\
\hline $0.72-0.90$ & 0.792 & 96.0 & $0.91 \pm 0.04 \pm 0.10$ & 0.792 & 111.7 & $0.21 \pm 0.02 \pm 0.04$ \\
\hline $0.90-1.25$ & 1.018 & 95.9 & $0.19 \pm 0.02 \pm 0.04$ & 1.012 & 111.8 & $0.03 \pm 0.01 \pm 0.02$ \\
\hline
\end{tabular}

error on the proton energy loss in materials between the interaction vertex and the TPC volume leads to a momentum change larger than $2 \%$. Pion cross-sections are not given if pions are separated from protons by less than twice the timeof-flight resolution.

The data given in Tables $2-10$ are available in ASCII format in [21].
We refrain from presenting the wealth of cross-section data also in the form of plots. We limit ourselves to three representative figures that show the inclusive cross-sections

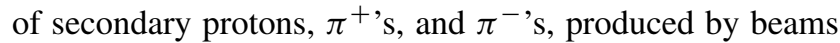
of protons, $\pi^{+}$'s, and $\pi^{-}$'s. We chose a polar-angle range that permits a good comparison of our results with published results from other experiments. 
Table 3 Double-differential inclusive cross-section $\mathrm{d}^{2} \sigma / \mathrm{d} p \mathrm{~d} \Omega[\mathrm{mb} /(\mathrm{GeV} / \mathrm{c}$ sr $)]$ of the production of $\pi^{+}$'s in $\mathrm{p}+\mathrm{Be} \rightarrow \pi^{+}+\mathrm{X}$ interactions with $+8.9 \mathrm{GeV} / c$ beam momentum; the first error is statistical, the second systematic; $p_{\mathrm{T}}$ in $\mathrm{GeV} / c$, polar angle $\theta$ in degrees

\begin{tabular}{|c|c|c|c|c|c|c|}
\hline \multirow[t]{2}{*}{$p_{\mathrm{T}}$} & \multicolumn{3}{|c|}{$20<\theta<30$} & \multicolumn{3}{|c|}{$30<\theta<40$} \\
\hline & $\left\langle p_{\mathrm{T}}\right\rangle$ & $\langle\theta\rangle$ & $\mathrm{d}^{2} \sigma / \mathrm{d} p \mathrm{~d} \Omega$ & $\left\langle p_{\mathrm{T}}\right\rangle$ & $\langle\theta\rangle$ & $\mathrm{d}^{2} \sigma / \mathrm{d} p \mathrm{~d} \Omega$ \\
\hline $0.10-0.13$ & 0.116 & 24.8 & $53.94 \pm 1.04 \pm 3.98$ & 0.116 & 34.7 & $37.12 \pm 0.84 \pm 2.54$ \\
\hline $0.13-0.16$ & 0.146 & 24.5 & $68.32 \pm 1.08 \pm 3.91$ & 0.146 & 34.7 & $48.46 \pm 0.92 \pm 2.53$ \\
\hline $0.16-0.20$ & 0.180 & 24.7 & $80.65 \pm 0.98 \pm 3.65$ & 0.180 & 34.8 & $51.60 \pm 0.78 \pm 2.26$ \\
\hline $0.20-0.24$ & 0.220 & 24.7 & $83.11 \pm 0.98 \pm 3.08$ & 0.220 & 34.7 & $55.60 \pm 0.80 \pm 2.10$ \\
\hline $0.24-0.30$ & 0.269 & 24.7 & $76.59 \pm 0.76 \pm 2.44$ & 0.269 & 34.7 & $51.20 \pm 0.61 \pm 1.60$ \\
\hline $0.30-0.36$ & 0.329 & 24.7 & $63.92 \pm 0.69 \pm 1.83$ & 0.329 & 34.7 & $43.01 \pm 0.56 \pm 1.19$ \\
\hline $0.36-0.42$ & 0.389 & 24.7 & $51.22 \pm 0.61 \pm 1.46$ & 0.389 & 34.7 & $35.38 \pm 0.51 \pm 0.96$ \\
\hline $0.42-0.50$ & 0.458 & 24.7 & $39.63 \pm 0.46 \pm 1.35$ & 0.458 & 34.7 & $27.21 \pm 0.38 \pm 0.83$ \\
\hline $0.50-0.60$ & 0.547 & 24.7 & $24.72 \pm 0.31 \pm 1.18$ & 0.547 & 34.7 & $17.24 \pm 0.27 \pm 0.72$ \\
\hline $0.60-0.72$ & 0.655 & 24.8 & $14.28 \pm 0.21 \pm 1.02$ & 0.654 & 34.6 & $9.97 \pm 0.18 \pm 0.62$ \\
\hline $0.72-0.90$ & & & & 0.794 & 34.6 & $4.41 \pm 0.09 \pm 0.44$ \\
\hline \multirow[t]{2}{*}{$p_{\mathrm{T}}$} & \multicolumn{3}{|c|}{$40<\theta<50$} & \multicolumn{3}{|c|}{$50<\theta<60$} \\
\hline & $\overline{\left\langle p_{\mathrm{T}}\right\rangle}$ & $\langle\theta\rangle$ & $\mathrm{d}^{2} \sigma / \mathrm{d} p \mathrm{~d} \Omega$ & $\left\langle p_{\mathrm{T}}\right\rangle$ & $\langle\theta\rangle$ & $\mathrm{d}^{2} \sigma / \mathrm{d} p \mathrm{~d} \Omega$ \\
\hline $0.10-0.13$ & 0.116 & 44.9 & $28.16 \pm 0.73 \pm 1.94$ & & & \\
\hline $0.13-0.16$ & 0.145 & 44.9 & $34.68 \pm 0.76 \pm 1.83$ & 0.146 & 54.9 & $25.72 \pm 0.64 \pm 1.41$ \\
\hline $0.16-0.20$ & 0.180 & 44.8 & $38.77 \pm 0.68 \pm 1.72$ & 0.180 & 54.8 & $30.54 \pm 0.60 \pm 1.36$ \\
\hline $0.20-0.24$ & 0.220 & 44.7 & $40.38 \pm 0.68 \pm 1.52$ & 0.220 & 54.8 & $29.38 \pm 0.57 \pm 1.16$ \\
\hline $0.24-0.30$ & 0.269 & 44.7 & $36.11 \pm 0.52 \pm 1.14$ & 0.269 & 54.7 & $26.24 \pm 0.44 \pm 0.84$ \\
\hline $0.30-0.36$ & 0.329 & 44.8 & $29.85 \pm 0.47 \pm 0.84$ & 0.329 & 54.7 & $21.36 \pm 0.39 \pm 0.60$ \\
\hline $0.36-0.42$ & 0.389 & 44.7 & $25.01 \pm 0.43 \pm 0.69$ & 0.389 & 54.8 & $15.57 \pm 0.33 \pm 0.45$ \\
\hline $0.42-0.50$ & 0.458 & 44.6 & $18.06 \pm 0.31 \pm 0.56$ & 0.457 & 54.7 & $11.91 \pm 0.25 \pm 0.39$ \\
\hline $0.50-0.60$ & 0.547 & 44.6 & $11.65 \pm 0.22 \pm 0.47$ & 0.546 & 54.6 & $8.23 \pm 0.19 \pm 0.35$ \\
\hline $0.60-0.72$ & 0.655 & 44.6 & $7.10 \pm 0.16 \pm 0.40$ & 0.654 & 54.7 & $4.45 \pm 0.12 \pm 0.26$ \\
\hline $0.72-0.90$ & 0.795 & 44.5 & $3.18 \pm 0.08 \pm 0.28$ & 0.793 & 54.3 & $1.96 \pm 0.06 \pm 0.17$ \\
\hline $0.90-1.25$ & & & & 1.027 & 54.2 & $0.36 \pm 0.02 \pm 0.05$ \\
\hline \multirow[t]{2}{*}{$p_{\mathrm{T}}$} & \multicolumn{3}{|c|}{$60<\theta<75$} & \multicolumn{3}{|c|}{$75<\theta<90$} \\
\hline & $\left\langle p_{\mathrm{T}}\right\rangle$ & $\langle\theta\rangle$ & $\mathrm{d}^{2} \sigma / \mathrm{d} p \mathrm{~d} \Omega$ & $\left\langle p_{\mathrm{T}}\right\rangle$ & $\langle\theta\rangle$ & $\mathrm{d}^{2} \sigma / \mathrm{d} p \mathrm{~d} \Omega$ \\
\hline $0.13-0.16$ & 0.146 & 67.1 & $20.46 \pm 0.46 \pm 1.13$ & 0.145 & 82.5 & $16.41 \pm 0.41 \pm 0.92$ \\
\hline $0.16-0.20$ & 0.180 & 67.3 & $23.19 \pm 0.41 \pm 1.00$ & 0.180 & 82.2 & $17.47 \pm 0.35 \pm 0.75$ \\
\hline $0.20-0.24$ & 0.219 & 67.2 & $21.65 \pm 0.40 \pm 0.86$ & 0.219 & 82.0 & $15.72 \pm 0.33 \pm 0.63$ \\
\hline $0.24-0.30$ & 0.269 & 67.0 & $17.35 \pm 0.29 \pm 0.55$ & 0.268 & 82.0 & $11.83 \pm 0.24 \pm 0.41$ \\
\hline $0.30-0.36$ & 0.329 & 66.9 & $13.61 \pm 0.26 \pm 0.39$ & 0.328 & 81.9 & $8.09 \pm 0.20 \pm 0.26$ \\
\hline $0.36-0.42$ & 0.388 & 66.8 & $9.72 \pm 0.21 \pm 0.30$ & 0.388 & 81.9 & $5.53 \pm 0.16 \pm 0.21$ \\
\hline $0.42-0.50$ & 0.457 & 66.7 & $7.24 \pm 0.16 \pm 0.27$ & 0.458 & 81.9 & $4.43 \pm 0.13 \pm 0.20$ \\
\hline $0.50-0.60$ & 0.546 & 66.7 & $4.69 \pm 0.12 \pm 0.24$ & 0.543 & 81.9 & $2.40 \pm 0.08 \pm 0.15$ \\
\hline $0.60-0.72$ & 0.653 & 66.3 & $2.51 \pm 0.08 \pm 0.17$ & 0.654 & 81.3 & $1.06 \pm 0.05 \pm 0.09$ \\
\hline $0.72-0.90$ & 0.792 & 66.4 & $0.89 \pm 0.03 \pm 0.09$ & 0.791 & 81.3 & $0.27 \pm 0.02 \pm 0.03$ \\
\hline $0.90-1.25$ & 1.010 & 66.0 & $0.13 \pm 0.01 \pm 0.02$ & 1.002 & 81.4 & $0.03 \pm 0.01 \pm 0.01$ \\
\hline \multirow[t]{2}{*}{$p_{\mathrm{T}}$} & \multicolumn{3}{|c|}{$90<\theta<105$} & \multicolumn{3}{|c|}{$105<\theta<125$} \\
\hline & $\left\langle p_{\mathrm{T}}\right\rangle$ & $\langle\theta\rangle$ & $\mathrm{d}^{2} \sigma / \mathrm{d} p \mathrm{~d} \Omega$ & $\overline{\left\langle p_{\mathrm{T}}\right\rangle}$ & $\langle\theta\rangle$ & $\mathrm{d}^{2} \sigma / \mathrm{d} p \mathrm{~d} \Omega$ \\
\hline $0.13-0.16$ & 0.145 & 97.4 & $14.32 \pm 0.37 \pm 0.79$ & 0.145 & 114.9 & $11.20 \pm 0.27 \pm 0.53$ \\
\hline $0.16-0.20$ & 0.179 & 97.3 & $13.31 \pm 0.29 \pm 0.54$ & 0.179 & 114.0 & $9.75 \pm 0.22 \pm 0.39$ \\
\hline $0.20-0.24$ & 0.219 & 97.2 & $11.85 \pm 0.28 \pm 0.43$ & 0.219 & 113.7 & $7.40 \pm 0.20 \pm 0.29$ \\
\hline $0.24-0.30$ & 0.268 & 96.9 & $7.55 \pm 0.19 \pm 0.26$ & 0.267 & 113.6 & $4.60 \pm 0.13 \pm 0.19$ \\
\hline $0.30-0.36$ & 0.328 & 96.8 & $5.25 \pm 0.16 \pm 0.20$ & 0.327 & 113.6 & $2.73 \pm 0.10 \pm 0.14$ \\
\hline $0.36-0.42$ & 0.387 & 96.6 & $3.63 \pm 0.14 \pm 0.18$ & 0.387 & 113.2 & $1.55 \pm 0.07 \pm 0.10$ \\
\hline $0.42-0.50$ & 0.457 & 96.4 & $2.10 \pm 0.09 \pm 0.14$ & 0.455 & 112.8 & $0.70 \pm 0.04 \pm 0.06$ \\
\hline $0.50-0.60$ & 0.541 & 96.2 & $1.07 \pm 0.06 \pm 0.09$ & 0.536 & 111.5 & $0.22 \pm 0.02 \pm 0.03$ \\
\hline $0.60-0.72$ & 0.650 & 95.9 & $0.35 \pm 0.03 \pm 0.04$ & 0.655 & 111.2 & $0.07 \pm 0.02 \pm 0.02$ \\
\hline $0.72-0.90$ & 0.788 & 95.5 & $0.09 \pm 0.02 \pm 0.02$ & & & \\
\hline
\end{tabular}


Table 4 Double-differential inclusive cross-section $\mathrm{d}^{2} \sigma / \mathrm{d} p \mathrm{~d} \Omega[\mathrm{mb} /(\mathrm{GeV} / \mathrm{cs})]$ of the production of $\pi^{-}$'s in $\mathrm{p}+\mathrm{Be} \rightarrow \pi^{-}+\mathrm{X}$ interactions with $+8.9 \mathrm{GeV} / c$ beam momentum; the first error is statistical, the second systematic; $p_{\mathrm{T}}$ in $\mathrm{GeV} / c$, polar angle $\theta$ in degrees

\begin{tabular}{|c|c|c|c|c|c|c|}
\hline \multirow[t]{2}{*}{$p_{\mathrm{T}}$} & \multicolumn{3}{|c|}{$20<\theta<30$} & \multicolumn{3}{|c|}{$30<\theta<40$} \\
\hline & $\overline{\left\langle p_{\mathrm{T}}\right\rangle}$ & $\langle\theta\rangle$ & $\mathrm{d}^{2} \sigma / \mathrm{d} p \mathrm{~d} \Omega$ & $\overline{\left\langle p_{\mathrm{T}}\right\rangle}$ & $\langle\theta\rangle$ & $\mathrm{d}^{2} \sigma / \mathrm{d} p \mathrm{~d} \Omega$ \\
\hline $0.10-0.13$ & 0.116 & 24.7 & $52.88 \pm 0.99 \pm 3.67$ & 0.116 & 34.7 & $37.22 \pm 0.80 \pm 2.54$ \\
\hline $0.13-0.16$ & 0.145 & 24.8 & $68.86 \pm 1.07 \pm 3.72$ & 0.145 & 34.7 & $43.73 \pm 0.81 \pm 2.33$ \\
\hline $0.16-0.20$ & 0.180 & 24.7 & $73.99 \pm 0.92 \pm 3.23$ & 0.180 & 34.7 & $51.74 \pm 0.77 \pm 2.28$ \\
\hline $0.20-0.24$ & 0.220 & 24.8 & $70.71 \pm 0.89 \pm 2.59$ & 0.220 & 34.6 & $50.06 \pm 0.74 \pm 1.85$ \\
\hline $0.24-0.30$ & 0.269 & 24.8 & $63.44 \pm 0.68 \pm 1.95$ & 0.269 & 34.7 & $45.71 \pm 0.57 \pm 1.41$ \\
\hline $0.30-0.36$ & 0.329 & 24.8 & $50.19 \pm 0.60 \pm 1.37$ & 0.329 & 34.8 & $38.12 \pm 0.52 \pm 1.04$ \\
\hline $0.36-0.42$ & 0.389 & 24.8 & $39.48 \pm 0.54 \pm 1.10$ & 0.389 & 34.7 & $30.08 \pm 0.46 \pm 0.83$ \\
\hline $0.42-0.50$ & 0.458 & 24.8 & $28.54 \pm 0.39 \pm 0.93$ & 0.457 & 34.7 & $20.57 \pm 0.33 \pm 0.66$ \\
\hline $0.50-0.60$ & 0.545 & 24.8 & $17.69 \pm 0.28 \pm 0.77$ & 0.545 & 34.8 & $13.29 \pm 0.23 \pm 0.56$ \\
\hline $0.60-0.72$ & 0.654 & 24.9 & $9.49 \pm 0.18 \pm 0.56$ & 0.653 & 34.8 & $7.13 \pm 0.15 \pm 0.41$ \\
\hline $0.72-0.90$ & & & & 0.792 & 35.0 & $2.79 \pm 0.08 \pm 0.22$ \\
\hline \multirow[t]{2}{*}{$p_{\mathrm{T}}$} & \multicolumn{3}{|c|}{$40<\theta<50$} & \multicolumn{3}{|c|}{$50<\theta<60$} \\
\hline & $\overline{\left\langle p_{\mathrm{T}}\right\rangle}$ & $\langle\theta\rangle$ & $\mathrm{d}^{2} \sigma / \mathrm{d} p \mathrm{~d} \Omega$ & $\overline{\left\langle p_{\mathrm{T}}\right\rangle}$ & $\langle\theta\rangle$ & $\mathrm{d}^{2} \sigma / \mathrm{d} p \mathrm{~d} \Omega$ \\
\hline $0.10-0.13$ & 0.116 & 44.9 & $26.16 \pm 0.66 \pm 1.84$ & & & \\
\hline $0.13-0.16$ & 0.145 & 44.8 & $32.69 \pm 0.70 \pm 1.74$ & 0.145 & 54.9 & $27.00 \pm 0.62 \pm 1.51$ \\
\hline $0.16-0.20$ & 0.180 & 44.9 & $35.72 \pm 0.63 \pm 1.58$ & 0.180 & 54.8 & $30.60 \pm 0.58 \pm 1.38$ \\
\hline $0.20-0.24$ & 0.220 & 44.7 & $35.26 \pm 0.61 \pm 1.32$ & 0.220 & 54.8 & $29.13 \pm 0.57 \pm 1.10$ \\
\hline $0.24-0.30$ & 0.269 & 44.7 & $31.62 \pm 0.47 \pm 0.98$ & 0.269 & 54.7 & $24.82 \pm 0.43 \pm 0.77$ \\
\hline $0.30-0.36$ & 0.329 & 44.7 & $27.66 \pm 0.45 \pm 0.76$ & 0.329 & 54.8 & $18.64 \pm 0.36 \pm 0.52$ \\
\hline $0.36-0.42$ & 0.389 & 44.8 & $20.94 \pm 0.38 \pm 0.60$ & 0.389 & 54.6 & $14.03 \pm 0.30 \pm 0.42$ \\
\hline $0.42-0.50$ & 0.457 & 44.8 & $14.98 \pm 0.27 \pm 0.50$ & 0.457 & 54.7 & $10.00 \pm 0.22 \pm 0.35$ \\
\hline $0.50-0.60$ & 0.546 & 44.7 & $8.98 \pm 0.18 \pm 0.40$ & 0.545 & 54.6 & $6.11 \pm 0.15 \pm 0.28$ \\
\hline $0.60-0.72$ & 0.653 & 44.9 & $5.38 \pm 0.13 \pm 0.33$ & 0.652 & 54.7 & $3.33 \pm 0.10 \pm 0.21$ \\
\hline $0.72-0.90$ & 0.793 & 44.8 & $1.79 \pm 0.06 \pm 0.15$ & 0.793 & 54.4 & $1.35 \pm 0.05 \pm 0.12$ \\
\hline $0.90-1.25$ & & & & 1.008 & 54.3 & $0.23 \pm 0.02 \pm 0.03$ \\
\hline \multirow[t]{2}{*}{$p_{\mathrm{T}}$} & \multicolumn{3}{|c|}{$60<\theta<75$} & \multicolumn{3}{|c|}{$75<\theta<90$} \\
\hline & $\overline{\left\langle p_{\mathrm{T}}\right\rangle}$ & $\langle\theta\rangle$ & $\mathrm{d}^{2} \sigma / \mathrm{d} p \mathrm{~d} \Omega$ & $\overline{\left\langle p_{\mathrm{T}}\right\rangle}$ & $\langle\theta\rangle$ & $\mathrm{d}^{2} \sigma / \mathrm{d} p \mathrm{~d} \Omega$ \\
\hline $0.13-0.16$ & 0.145 & 67.2 & $21.82 \pm 0.46 \pm 1.18$ & 0.145 & 82.4 & $15.38 \pm 0.37 \pm 0.86$ \\
\hline $0.16-0.20$ & 0.180 & 67.2 & $22.68 \pm 0.39 \pm 0.95$ & 0.180 & 82.5 & $16.85 \pm 0.32 \pm 0.69$ \\
\hline $0.20-0.24$ & 0.219 & 67.3 & $21.05 \pm 0.38 \pm 0.76$ & 0.219 & 82.3 & $15.18 \pm 0.32 \pm 0.55$ \\
\hline $0.24-0.30$ & 0.269 & 66.9 & $15.96 \pm 0.27 \pm 0.47$ & 0.268 & 82.0 & $10.95 \pm 0.22 \pm 0.33$ \\
\hline $0.30-0.36$ & 0.329 & 66.8 & $12.48 \pm 0.23 \pm 0.36$ & 0.327 & 81.9 & $7.40 \pm 0.18 \pm 0.24$ \\
\hline $0.36-0.42$ & 0.389 & 66.8 & $9.45 \pm 0.20 \pm 0.30$ & 0.388 & 82.0 & $4.85 \pm 0.14 \pm 0.19$ \\
\hline $0.42-0.50$ & 0.456 & 66.6 & $6.04 \pm 0.13 \pm 0.24$ & 0.457 & 81.5 & $3.58 \pm 0.11 \pm 0.18$ \\
\hline $0.50-0.60$ & 0.547 & 66.7 & $3.85 \pm 0.10 \pm 0.20$ & 0.544 & 81.5 & $1.82 \pm 0.07 \pm 0.12$ \\
\hline $0.60-0.72$ & 0.651 & 66.5 & $1.73 \pm 0.06 \pm 0.12$ & 0.649 & 81.3 & $0.97 \pm 0.05 \pm 0.09$ \\
\hline $0.72-0.90$ & 0.793 & 66.6 & $0.69 \pm 0.03 \pm 0.07$ & 0.782 & 81.4 & $0.25 \pm 0.02 \pm 0.03$ \\
\hline $0.90-1.25$ & 1.021 & 66.3 & $0.10 \pm 0.01 \pm 0.02$ & 1.037 & 80.4 & $0.04 \pm 0.01 \pm 0.01$ \\
\hline \multirow[t]{2}{*}{$p_{\mathrm{T}}$} & \multicolumn{3}{|c|}{$90<\theta<105$} & \multicolumn{3}{|c|}{$105<\theta<125$} \\
\hline & $\overline{\left\langle p_{\mathrm{T}}\right\rangle}$ & $\langle\theta\rangle$ & $\mathrm{d}^{2} \sigma / \mathrm{d} p \mathrm{~d} \Omega$ & $\overline{\left\langle p_{\mathrm{T}}\right\rangle}$ & $\langle\theta\rangle$ & $\mathrm{d}^{2} \sigma / \mathrm{d} p \mathrm{~d} \Omega$ \\
\hline $0.13-0.16$ & 0.145 & 97.6 & $15.03 \pm 0.36 \pm 0.83$ & 0.145 & 114.7 & $11.38 \pm 0.26 \pm 0.53$ \\
\hline $0.16-0.20$ & 0.179 & 97.3 & $14.31 \pm 0.30 \pm 0.57$ & 0.179 & 114.1 & $9.10 \pm 0.19 \pm 0.32$ \\
\hline $0.20-0.24$ & 0.219 & 97.0 & $11.02 \pm 0.25 \pm 0.39$ & 0.218 & 113.9 & $6.71 \pm 0.17 \pm 0.24$ \\
\hline $0.24-0.30$ & 0.267 & 96.8 & $7.66 \pm 0.18 \pm 0.25$ & 0.266 & 113.4 & $3.92 \pm 0.11 \pm 0.17$ \\
\hline $0.30-0.36$ & 0.329 & 97.1 & $4.97 \pm 0.15 \pm 0.20$ & 0.326 & 113.7 & $2.45 \pm 0.09 \pm 0.14$ \\
\hline $0.36-0.42$ & 0.387 & 96.7 & $2.91 \pm 0.11 \pm 0.16$ & 0.387 & 113.2 & $1.44 \pm 0.07 \pm 0.11$ \\
\hline $0.42-0.50$ & 0.454 & 96.6 & $1.88 \pm 0.08 \pm 0.13$ & 0.454 & 112.5 & $0.70 \pm 0.04 \pm 0.07$ \\
\hline $0.50-0.60$ & 0.546 & 96.7 & $0.82 \pm 0.05 \pm 0.08$ & 0.539 & 111.3 & $0.22 \pm 0.02 \pm 0.03$ \\
\hline $0.60-0.72$ & 0.649 & 95.7 & $0.33 \pm 0.03 \pm 0.04$ & 0.636 & 110.1 & $0.05 \pm 0.01 \pm 0.02$ \\
\hline $0.72-0.90$ & 0.780 & 96.8 & $0.08 \pm 0.02 \pm 0.02$ & & & \\
\hline $0.90-1.25$ & & & & 1.020 & 114.1 & $0.04 \pm 0.02 \pm 0.02$ \\
\hline
\end{tabular}


Table 5 Double-differential inclusive cross-section $\mathrm{d}^{2} \sigma / \mathrm{d} p \mathrm{~d} \Omega[\mathrm{mb} /(\mathrm{GeV} / c \mathrm{sr})]$ of the production of protons in $\pi^{+}+\mathrm{Be} \rightarrow \mathrm{p}+\mathrm{X}$ interactions with $+8.9 \mathrm{GeV} / c$ beam momentum; the first error is statistical, the second systematic; $p_{\mathrm{T}}$ in $\mathrm{GeV} / c$, polar angle $\theta$ in degrees

\begin{tabular}{|c|c|c|c|c|c|c|}
\hline \multirow[t]{2}{*}{$p_{\mathrm{T}}$} & \multicolumn{3}{|c|}{$20<\theta<30$} & \multicolumn{3}{|c|}{$30<\theta<40$} \\
\hline & $\left\langle p_{\mathrm{T}}\right\rangle$ & $\langle\theta\rangle$ & $\mathrm{d}^{2} \sigma / \mathrm{d} p \mathrm{~d} \Omega$ & $\left\langle p_{\mathrm{T}}\right\rangle$ & $\langle\theta\rangle$ & $\mathrm{d}^{2} \sigma / \mathrm{d} p \mathrm{~d} \Omega$ \\
\hline $0.20-0.24$ & 0.220 & 24.8 & $34.44 \pm 0.76 \pm 1.62$ & & & \\
\hline $0.24-0.30$ & 0.269 & 25.1 & $36.77 \pm 0.64 \pm 1.54$ & 0.270 & 34.9 & $32.90 \pm 0.66 \pm 1.30$ \\
\hline $0.30-0.36$ & 0.329 & 25.0 & $32.47 \pm 0.58 \pm 1.22$ & 0.330 & 34.9 & $30.81 \pm 0.56 \pm 1.07$ \\
\hline $0.36-0.42$ & 0.389 & 24.9 & $30.62 \pm 0.56 \pm 1.05$ & 0.389 & 34.9 & $27.98 \pm 0.54 \pm 0.89$ \\
\hline $0.42-0.50$ & 0.458 & 25.0 & $28.19 \pm 0.47 \pm 0.90$ & 0.459 & 34.9 & $25.72 \pm 0.45 \pm 0.78$ \\
\hline $0.50-0.60$ & 0.547 & 24.9 & $23.47 \pm 0.37 \pm 0.77$ & 0.548 & 34.9 & $20.87 \pm 0.36 \pm 0.65$ \\
\hline $0.60-0.72$ & 0.656 & 24.9 & $19.42 \pm 0.31 \pm 0.74$ & 0.657 & 34.8 & $15.84 \pm 0.28 \pm 0.59$ \\
\hline $0.72-0.90$ & & & & 0.802 & 34.8 & $10.53 \pm 0.19 \pm 0.54$ \\
\hline \multirow[t]{2}{*}{$p_{\mathrm{T}}$} & \multicolumn{3}{|c|}{$40<\theta<50$} & \multicolumn{3}{|c|}{$50<\theta<60$} \\
\hline & $\overline{\left\langle p_{\mathrm{T}}\right\rangle}$ & $\langle\theta\rangle$ & $\mathrm{d}^{2} \sigma / \mathrm{d} p \mathrm{~d} \Omega$ & $\overline{\left\langle p_{\mathrm{T}}\right\rangle}$ & $\langle\theta\rangle$ & $\mathrm{d}^{2} \sigma / \mathrm{d} p \mathrm{~d} \Omega$ \\
\hline $0.30-0.36$ & 0.330 & 45.0 & $30.20 \pm 0.55 \pm 1.00$ & & & \\
\hline $0.36-0.42$ & 0.389 & 45.0 & $28.76 \pm 0.55 \pm 0.85$ & 0.389 & 55.0 & $26.46 \pm 0.51 \pm 0.75$ \\
\hline $0.42-0.50$ & 0.459 & 45.0 & $23.87 \pm 0.44 \pm 0.69$ & 0.458 & 54.8 & $20.94 \pm 0.40 \pm 0.61$ \\
\hline $0.50-0.60$ & 0.548 & 44.9 & $17.94 \pm 0.34 \pm 0.58$ & 0.547 & 54.9 & $15.89 \pm 0.32 \pm 0.55$ \\
\hline $0.60-0.72$ & 0.656 & 44.8 & $13.30 \pm 0.27 \pm 0.51$ & 0.657 & 54.9 & $11.27 \pm 0.26 \pm 0.48$ \\
\hline $0.72-0.90$ & 0.800 & 44.9 & $8.01 \pm 0.18 \pm 0.42$ & 0.799 & 54.8 & $5.75 \pm 0.15 \pm 0.33$ \\
\hline $0.90-1.25$ & 1.031 & 44.7 & $2.74 \pm 0.07 \pm 0.22$ & 1.027 & 54.8 & $1.69 \pm 0.06 \pm 0.15$ \\
\hline \multirow[t]{2}{*}{$p_{\mathrm{T}}$} & \multicolumn{3}{|c|}{$60<\theta<75$} & \multicolumn{3}{|c|}{$75<\theta<90$} \\
\hline & $\left\langle p_{\mathrm{T}}\right\rangle$ & $\langle\theta\rangle$ & $\mathrm{d}^{2} \sigma / \mathrm{d} p \mathrm{~d} \Omega$ & $\left\langle p_{\mathrm{T}}\right\rangle$ & $\langle\theta\rangle$ & $\mathrm{d}^{2} \sigma / \mathrm{d} p \mathrm{~d} \Omega$ \\
\hline $0.36-0.42$ & 0.389 & 67.4 & $22.14 \pm 0.37 \pm 0.72$ & & & \\
\hline $0.42-0.50$ & 0.458 & 67.3 & $20.09 \pm 0.32 \pm 0.58$ & 0.457 & 81.9 & $14.52 \pm 0.27 \pm 0.53$ \\
\hline $0.50-0.60$ & 0.547 & 67.2 & $14.32 \pm 0.25 \pm 0.51$ & 0.545 & 81.8 & $9.33 \pm 0.20 \pm 0.42$ \\
\hline $0.60-0.72$ & 0.655 & 67.2 & $8.69 \pm 0.18 \pm 0.44$ & 0.654 & 81.7 & $4.55 \pm 0.13 \pm 0.30$ \\
\hline $0.72-0.90$ & 0.796 & 66.7 & $4.07 \pm 0.10 \pm 0.29$ & 0.798 & 81.5 & $2.00 \pm 0.07 \pm 0.18$ \\
\hline $0.90-1.25$ & 1.026 & 66.3 & $0.96 \pm 0.04 \pm 0.11$ & 1.018 & 81.5 & $0.47 \pm 0.03 \pm 0.06$ \\
\hline \multirow[t]{2}{*}{$p_{\mathrm{T}}$} & \multicolumn{3}{|c|}{$90<\theta<105$} & \multicolumn{3}{|c|}{$105<\theta<125$} \\
\hline & $\left\langle p_{\mathrm{T}}\right\rangle$ & $\langle\theta\rangle$ & $\mathrm{d}^{2} \sigma / \mathrm{d} p \mathrm{~d} \Omega$ & $\left\langle p_{\mathrm{T}}\right\rangle$ & $\langle\theta\rangle$ & $\mathrm{d}^{2} \sigma / \mathrm{d} p \mathrm{~d} \Omega$ \\
\hline $0.36-0.42$ & & & & 0.388 & 113.7 & $6.70 \pm 0.18 \pm 0.24$ \\
\hline $0.42-0.50$ & 0.456 & 96.9 & $8.60 \pm 0.21 \pm 0.41$ & 0.457 & 113.5 & $4.45 \pm 0.13 \pm 0.20$ \\
\hline $0.50-0.60$ & 0.545 & 96.7 & $5.17 \pm 0.15 \pm 0.31$ & 0.544 & 112.8 & $2.26 \pm 0.09 \pm 0.17$ \\
\hline $0.60-0.72$ & 0.650 & 96.6 & $2.26 \pm 0.10 \pm 0.18$ & 0.647 & 112.7 & $0.71 \pm 0.05 \pm 0.08$ \\
\hline $0.72-0.90$ & 0.792 & 96.4 & $0.76 \pm 0.05 \pm 0.08$ & 0.793 & 112.1 & $0.22 \pm 0.02 \pm 0.04$ \\
\hline $0.90-1.25$ & 1.018 & 95.8 & $0.19 \pm 0.02 \pm 0.03$ & 1.016 & 111.6 & $0.03 \pm 0.01 \pm 0.01$ \\
\hline
\end{tabular}

Figure 13 illustrates our measurement of the inclusive cross-sections $\mathrm{d}^{2} \sigma / \mathrm{d} p \mathrm{~d} \Omega$ of proton and $\pi^{ \pm}$production by $+8.9 \mathrm{GeV} / c$ incoming protons, in the polar angle range $20^{\circ}<\theta<30^{\circ}$.

Figure 14 illustrates our measurement of the inclusive cross-sections $\mathrm{d}^{2} \sigma / \mathrm{d} p \mathrm{~d} \Omega$ of proton and $\pi^{ \pm}$production by $+8.9 \mathrm{GeV} / c$ incoming $\pi^{+}$'s, in the polar angle range $20^{\circ}<$ $\theta<30^{\circ}$.

Figure 15 illustrates our measurement of the inclusive cross-sections $\mathrm{d}^{2} \sigma / \mathrm{d} p \mathrm{~d} \Omega$ of proton and $\pi^{ \pm}$production by
$-8.0 \mathrm{GeV} / c$ incoming $\pi^{-}$'s, in the polar angle range $20^{\circ}<$ $\theta<30^{\circ}$.

\section{Comparison with other experimental results}

We compare our $+8.9 \mathrm{GeV} / c$ Be cross-sections with those from other experiments and with the results obtained by the HARP Collaboration from the same data that we analysed. The data of the other experiments are from E802 and E910 
Table 6 Double-differential inclusive cross-section $\mathrm{d}^{2} \sigma / \mathrm{d} p \mathrm{~d} \Omega[\mathrm{mb} /(\mathrm{GeV} / \mathrm{csr})]$ of the production of $\pi^{+}$'s in $\pi^{+}+\mathrm{Be} \rightarrow \pi^{+}+\mathrm{X}$ interactions with $+8.9 \mathrm{GeV} / c$ beam momentum; the first error is statistical, the second systematic; $p_{\mathrm{T}}$ in $\mathrm{GeV} / c$, polar angle $\theta$ in degrees

\begin{tabular}{|c|c|c|c|c|c|c|}
\hline \multirow[t]{2}{*}{$p_{\mathrm{T}}$} & \multicolumn{3}{|c|}{$20<\theta<30$} & \multicolumn{3}{|c|}{$30<\theta<40$} \\
\hline & $\left\langle p_{\mathrm{T}}\right\rangle$ & $\langle\theta\rangle$ & $\mathrm{d}^{2} \sigma / \mathrm{d} p \mathrm{~d} \Omega$ & $\left\langle p_{\mathrm{T}}\right\rangle$ & $\langle\theta\rangle$ & $\mathrm{d}^{2} \sigma / \mathrm{d} p \mathrm{~d} \Omega$ \\
\hline $0.10-0.13$ & 0.115 & 24.6 & $52.73 \pm 1.28 \pm 3.46$ & 0.116 & 34.7 & $38.43 \pm 1.06 \pm 2.58$ \\
\hline $0.13-0.16$ & 0.145 & 24.6 & $68.39 \pm 1.35 \pm 3.63$ & 0.146 & 34.7 & $49.02 \pm 1.16 \pm 2.55$ \\
\hline $0.16-0.20$ & 0.180 & 24.6 & $85.35 \pm 1.26 \pm 3.81$ & 0.180 & 34.7 & $54.25 \pm 0.99 \pm 2.36$ \\
\hline $0.20-0.24$ & 0.220 & 24.7 & $90.83 \pm 1.28 \pm 3.47$ & 0.220 & 34.7 & $59.18 \pm 1.03 \pm 2.21$ \\
\hline $0.24-0.30$ & 0.270 & 24.6 & $86.94 \pm 1.01 \pm 2.86$ & 0.270 & 34.7 & $55.39 \pm 0.80 \pm 1.77$ \\
\hline $0.30-0.36$ & 0.329 & 24.7 & $78.97 \pm 0.96 \pm 2.38$ & 0.329 & 34.6 & $49.51 \pm 0.75 \pm 1.41$ \\
\hline $0.36-0.42$ & 0.389 & 24.6 & $65.31 \pm 0.86 \pm 1.97$ & 0.389 & 34.5 & $41.04 \pm 0.68 \pm 1.14$ \\
\hline $0.42-0.50$ & 0.458 & 24.6 & $50.82 \pm 0.66 \pm 1.77$ & 0.458 & 34.7 & $31.00 \pm 0.51 \pm 0.97$ \\
\hline $0.50-0.60$ & 0.546 & 24.6 & $34.19 \pm 0.47 \pm 1.65$ & 0.546 & 34.6 & $20.89 \pm 0.37 \pm 0.89$ \\
\hline $0.60-0.72$ & 0.653 & 24.5 & $20.15 \pm 0.32 \pm 1.44$ & 0.655 & 34.6 & $12.10 \pm 0.25 \pm 0.76$ \\
\hline $0.72-0.90$ & & & & 0.796 & 34.4 & $5.44 \pm 0.13 \pm 0.55$ \\
\hline \multirow[t]{2}{*}{$p_{\mathrm{T}}$} & \multicolumn{3}{|c|}{$40<\theta<50$} & \multicolumn{3}{|c|}{$50<\theta<60$} \\
\hline & $\overline{\left\langle p_{\mathrm{T}}\right\rangle}$ & $\langle\theta\rangle$ & $\mathrm{d}^{2} \sigma / \mathrm{d} p \mathrm{~d} \Omega$ & $\left\langle p_{\mathrm{T}}\right\rangle$ & $\langle\theta\rangle$ & $\mathrm{d}^{2} \sigma / \mathrm{d} p \mathrm{~d} \Omega$ \\
\hline $0.10-0.13$ & 0.116 & 44.8 & $27.23 \pm 0.89 \pm 1.89$ & & & \\
\hline $0.13-0.16$ & 0.145 & 45.0 & $34.43 \pm 0.95 \pm 1.80$ & 0.146 & 54.8 & $26.57 \pm 0.81 \pm 1.47$ \\
\hline $0.16-0.20$ & 0.180 & 44.7 & $40.25 \pm 0.86 \pm 1.76$ & 0.180 & 54.8 & $29.74 \pm 0.74 \pm 1.31$ \\
\hline $0.20-0.24$ & 0.220 & 44.7 & $41.13 \pm 0.86 \pm 1.55$ & 0.220 & 54.7 & $29.17 \pm 0.72 \pm 1.09$ \\
\hline $0.24-0.30$ & 0.269 & 44.8 & $37.59 \pm 0.66 \pm 1.21$ & 0.269 & 54.7 & $27.17 \pm 0.56 \pm 0.86$ \\
\hline $0.30-0.36$ & 0.329 & 44.7 & $31.47 \pm 0.60 \pm 0.91$ & 0.329 & 54.6 & $22.68 \pm 0.51 \pm 0.66$ \\
\hline $0.36-0.42$ & 0.389 & 44.6 & $26.25 \pm 0.55 \pm 0.75$ & 0.388 & 54.6 & $16.42 \pm 0.42 \pm 0.49$ \\
\hline $0.42-0.50$ & 0.458 & 44.6 & $19.54 \pm 0.41 \pm 0.62$ & 0.457 & 54.7 & $13.11 \pm 0.33 \pm 0.44$ \\
\hline $0.50-0.60$ & 0.545 & 44.6 & $13.17 \pm 0.29 \pm 0.53$ & 0.547 & 54.6 & $8.35 \pm 0.23 \pm 0.36$ \\
\hline $0.60-0.72$ & 0.654 & 44.4 & $7.69 \pm 0.20 \pm 0.44$ & 0.654 & 54.5 & $5.26 \pm 0.17 \pm 0.31$ \\
\hline $0.72-0.90$ & 0.793 & 44.5 & $3.72 \pm 0.11 \pm 0.32$ & 0.791 & 54.4 & $2.28 \pm 0.09 \pm 0.19$ \\
\hline $0.90-1.25$ & & & & 1.012 & 54.3 & $0.38 \pm 0.02 \pm 0.06$ \\
\hline \multirow[t]{2}{*}{$p_{\mathrm{T}}$} & \multicolumn{3}{|c|}{$60<\theta<75$} & \multicolumn{3}{|c|}{$75<\theta<90$} \\
\hline & $\left\langle p_{\mathrm{T}}\right\rangle$ & $\langle\theta\rangle$ & $\mathrm{d}^{2} \sigma / \mathrm{d} p \mathrm{~d} \Omega$ & $\left\langle p_{\mathrm{T}}\right\rangle$ & $\langle\theta\rangle$ & $\mathrm{d}^{2} \sigma / \mathrm{d} p \mathrm{~d} \Omega$ \\
\hline $0.13-0.16$ & 0.146 & 67.4 & $19.92 \pm 0.56 \pm 1.14$ & 0.146 & 82.6 & $16.19 \pm 0.51 \pm 1.00$ \\
\hline $0.16-0.20$ & 0.180 & 67.2 & $21.98 \pm 0.50 \pm 0.97$ & 0.180 & 82.2 & $17.37 \pm 0.44 \pm 0.81$ \\
\hline $0.20-0.24$ & 0.219 & 66.9 & $21.74 \pm 0.50 \pm 0.80$ & 0.219 & 82.2 & $16.33 \pm 0.43 \pm 0.59$ \\
\hline $0.24-0.30$ & 0.269 & 67.2 & $18.47 \pm 0.38 \pm 0.57$ & 0.268 & 81.9 & $11.54 \pm 0.29 \pm 0.35$ \\
\hline $0.30-0.36$ & 0.329 & 66.8 & $14.14 \pm 0.33 \pm 0.41$ & 0.328 & 82.1 & $8.72 \pm 0.26 \pm 0.27$ \\
\hline $0.36-0.42$ & 0.389 & 66.7 & $10.89 \pm 0.28 \pm 0.34$ & 0.389 & 81.9 & $5.93 \pm 0.20 \pm 0.22$ \\
\hline $0.42-0.50$ & 0.457 & 66.7 & $7.65 \pm 0.20 \pm 0.30$ & 0.458 & 81.8 & $4.88 \pm 0.17 \pm 0.23$ \\
\hline $0.50-0.60$ & 0.545 & 66.6 & $5.50 \pm 0.16 \pm 0.28$ & 0.546 & 81.8 & $2.80 \pm 0.11 \pm 0.17$ \\
\hline $0.60-0.72$ & 0.654 & 66.3 & $2.95 \pm 0.11 \pm 0.20$ & 0.653 & 81.7 & $1.30 \pm 0.07 \pm 0.11$ \\
\hline $0.72-0.90$ & 0.794 & 66.2 & $1.03 \pm 0.05 \pm 0.10$ & 0.785 & 81.0 & $0.43 \pm 0.03 \pm 0.05$ \\
\hline $0.90-1.25$ & 1.021 & 65.7 & $0.16 \pm 0.02 \pm 0.03$ & 1.007 & 81.2 & $0.05 \pm 0.01 \pm 0.01$ \\
\hline \multirow[t]{2}{*}{$p_{\mathrm{T}}$} & \multicolumn{3}{|c|}{$90<\theta<105$} & \multicolumn{3}{|c|}{$105<\theta<125$} \\
\hline & $\left\langle p_{\mathrm{T}}\right\rangle$ & $\langle\theta\rangle$ & $\mathrm{d}^{2} \sigma / \mathrm{d} p \mathrm{~d} \Omega$ & $\overline{\left\langle p_{\mathrm{T}}\right\rangle}$ & $\langle\theta\rangle$ & $\mathrm{d}^{2} \sigma / \mathrm{d} p \mathrm{~d} \Omega$ \\
\hline $0.13-0.16$ & 0.145 & 97.1 & $13.79 \pm 0.46 \pm 0.85$ & 0.145 & 114.8 & $10.53 \pm 0.33 \pm 0.66$ \\
\hline $0.16-0.20$ & 0.179 & 97.3 & $12.92 \pm 0.36 \pm 0.60$ & 0.179 & 114.5 & $9.68 \pm 0.27 \pm 0.48$ \\
\hline $0.20-0.24$ & 0.219 & 97.2 & $11.60 \pm 0.35 \pm 0.42$ & 0.219 & 114.0 & $7.56 \pm 0.25 \pm 0.27$ \\
\hline $0.24-0.30$ & 0.267 & 97.0 & $8.27 \pm 0.25 \pm 0.27$ & 0.267 & 113.5 & $5.03 \pm 0.17 \pm 0.19$ \\
\hline $0.30-0.36$ & 0.328 & 96.9 & $5.50 \pm 0.20 \pm 0.21$ & 0.327 & 113.7 & $2.86 \pm 0.13 \pm 0.15$ \\
\hline $0.36-0.42$ & 0.389 & 97.1 & $4.23 \pm 0.18 \pm 0.21$ & 0.387 & 113.6 & $1.71 \pm 0.10 \pm 0.12$ \\
\hline $0.42-0.50$ & 0.457 & 96.7 & $2.37 \pm 0.11 \pm 0.15$ & 0.457 & 112.4 & $0.94 \pm 0.06 \pm 0.08$ \\
\hline $0.50-0.60$ & 0.539 & 96.1 & $1.34 \pm 0.08 \pm 0.12$ & 0.541 & 111.9 & $0.31 \pm 0.03 \pm 0.04$ \\
\hline $0.60-0.72$ & 0.652 & 96.3 & $0.45 \pm 0.04 \pm 0.05$ & 0.650 & 111.9 & $0.12 \pm 0.02 \pm 0.02$ \\
\hline $0.72-0.90$ & 0.794 & 95.5 & $0.10 \pm 0.02 \pm 0.02$ & & & \\
\hline
\end{tabular}


Table 7 Double-differential inclusive cross-section $\mathrm{d}^{2} \sigma / \mathrm{d} p \mathrm{~d} \Omega[\mathrm{mb} /(\mathrm{GeV} / \mathrm{csr})]$ of the production of $\pi^{-}$'s in $\pi^{+}+\mathrm{Be} \rightarrow \pi^{-}+\mathrm{X}$ interactions with $+8.9 \mathrm{GeV} / c$ beam momentum; the first error is statistical, the second systematic; $p_{\mathrm{T}}$ in $\mathrm{GeV} / c$, polar angle $\theta$ in degrees

\begin{tabular}{|c|c|c|c|c|c|c|}
\hline \multirow[t]{2}{*}{$p_{\mathrm{T}}$} & \multicolumn{3}{|c|}{$20<\theta<30$} & \multicolumn{3}{|c|}{$30<\theta<40$} \\
\hline & $\left\langle p_{\mathrm{T}}\right\rangle$ & $\langle\theta\rangle$ & $\mathrm{d}^{2} \sigma / \mathrm{d} p \mathrm{~d} \Omega$ & $\left\langle p_{\mathrm{T}}\right\rangle$ & $\langle\theta\rangle$ & $\mathrm{d}^{2} \sigma / \mathrm{d} p \mathrm{~d} \Omega$ \\
\hline $0.10-0.13$ & 0.116 & 24.8 & $46.25 \pm 1.15 \pm 3.10$ & 0.116 & 34.8 & $33.42 \pm 0.94 \pm 2.33$ \\
\hline $0.13-0.16$ & 0.146 & 24.6 & $61.24 \pm 1.26 \pm 3.17$ & 0.145 & 34.6 & $36.42 \pm 0.92 \pm 1.93$ \\
\hline $0.16-0.20$ & 0.180 & 24.7 & $65.33 \pm 1.07 \pm 2.89$ & 0.180 & 34.6 & $43.91 \pm 0.88 \pm 1.95$ \\
\hline $0.20-0.24$ & 0.220 & 24.6 & $70.02 \pm 1.10 \pm 2.61$ & 0.220 & 34.6 & $44.85 \pm 0.87 \pm 1.69$ \\
\hline $0.24-0.30$ & 0.269 & 24.7 & $63.57 \pm 0.85 \pm 2.03$ & 0.270 & 34.7 & $42.49 \pm 0.69 \pm 1.35$ \\
\hline $0.30-0.36$ & 0.329 & 24.7 & $51.65 \pm 0.76 \pm 1.45$ & 0.329 & 34.7 & $35.06 \pm 0.62 \pm 0.99$ \\
\hline $0.36-0.42$ & 0.388 & 24.7 & $42.11 \pm 0.69 \pm 1.20$ & 0.389 & 34.6 & $28.74 \pm 0.56 \pm 0.81$ \\
\hline $0.42-0.50$ & 0.457 & 24.7 & $31.10 \pm 0.51 \pm 1.03$ & 0.458 & 34.7 & $19.88 \pm 0.40 \pm 0.65$ \\
\hline $0.50-0.60$ & 0.546 & 24.7 & $19.63 \pm 0.36 \pm 0.86$ & 0.546 & 34.8 & $12.89 \pm 0.29 \pm 0.55$ \\
\hline $0.60-0.72$ & 0.652 & 24.6 & $10.21 \pm 0.23 \pm 0.61$ & 0.652 & 34.8 & $6.87 \pm 0.19 \pm 0.41$ \\
\hline $0.72-0.90$ & & & & 0.793 & 34.7 & $2.89 \pm 0.10 \pm 0.24$ \\
\hline \multirow[t]{2}{*}{$p_{\mathrm{T}}$} & \multicolumn{3}{|c|}{$40<\theta<50$} & \multicolumn{3}{|c|}{$50<\theta<60$} \\
\hline & $\overline{\left\langle p_{\mathrm{T}}\right\rangle}$ & $\langle\theta\rangle$ & $\mathrm{d}^{2} \sigma / \mathrm{d} p \mathrm{~d} \Omega$ & $\left\langle p_{\mathrm{T}}\right\rangle$ & $\langle\theta\rangle$ & $\mathrm{d}^{2} \sigma / \mathrm{d} p \mathrm{~d} \Omega$ \\
\hline $0.10-0.13$ & 0.116 & 44.7 & $22.32 \pm 0.76 \pm 1.63$ & & & \\
\hline $0.13-0.16$ & 0.145 & 44.8 & $26.81 \pm 0.79 \pm 1.48$ & 0.145 & 54.9 & $20.72 \pm 0.68 \pm 1.34$ \\
\hline $0.16-0.20$ & 0.180 & 44.7 & $30.04 \pm 0.72 \pm 1.35$ & 0.181 & 54.8 & $23.25 \pm 0.63 \pm 1.07$ \\
\hline $0.20-0.24$ & 0.220 & 44.7 & $29.53 \pm 0.70 \pm 1.14$ & 0.220 & 54.7 & $24.07 \pm 0.64 \pm 0.92$ \\
\hline $0.24-0.30$ & 0.270 & 44.7 & $27.50 \pm 0.55 \pm 0.88$ & 0.269 & 54.8 & $20.77 \pm 0.49 \pm 0.66$ \\
\hline $0.30-0.36$ & 0.329 & 44.8 & $24.30 \pm 0.52 \pm 0.69$ & 0.329 & 54.6 & $16.27 \pm 0.41 \pm 0.49$ \\
\hline $0.36-0.42$ & 0.389 & 44.8 & $18.89 \pm 0.45 \pm 0.56$ & 0.388 & 54.7 & $11.66 \pm 0.34 \pm 0.36$ \\
\hline $0.42-0.50$ & 0.458 & 44.7 & $13.59 \pm 0.33 \pm 0.47$ & 0.457 & 54.7 & $9.07 \pm 0.26 \pm 0.37$ \\
\hline $0.50-0.60$ & 0.544 & 44.9 & $8.19 \pm 0.22 \pm 0.38$ & 0.546 & 54.8 & $5.79 \pm 0.18 \pm 0.30$ \\
\hline $0.60-0.72$ & 0.652 & 44.7 & $4.95 \pm 0.16 \pm 0.32$ & 0.653 & 54.6 & $2.94 \pm 0.12 \pm 0.20$ \\
\hline $0.72-0.90$ & 0.797 & 44.7 & $1.74 \pm 0.07 \pm 0.16$ & 0.793 & 54.6 & $1.29 \pm 0.07 \pm 0.12$ \\
\hline $0.90-1.25$ & & & & 1.020 & 54.4 & $0.20 \pm 0.02 \pm 0.03$ \\
\hline \multirow[t]{2}{*}{$p_{\mathrm{T}}$} & \multicolumn{3}{|c|}{$60<\theta<75$} & \multicolumn{3}{|c|}{$75<\theta<90$} \\
\hline & $\left\langle p_{\mathrm{T}}\right\rangle$ & $\langle\theta\rangle$ & $\mathrm{d}^{2} \sigma / \mathrm{d} p \mathrm{~d} \Omega$ & $\left\langle p_{\mathrm{T}}\right\rangle$ & $\langle\theta\rangle$ & $\mathrm{d}^{2} \sigma / \mathrm{d} p \mathrm{~d} \Omega$ \\
\hline $0.13-0.16$ & 0.145 & 67.2 & $16.30 \pm 0.50 \pm 0.98$ & 0.145 & 82.3 & $11.29 \pm 0.39 \pm 0.78$ \\
\hline $0.16-0.20$ & 0.180 & 67.4 & $17.40 \pm 0.43 \pm 0.81$ & 0.180 & 82.3 & $12.37 \pm 0.35 \pm 0.65$ \\
\hline $0.20-0.24$ & 0.220 & 67.2 & $16.22 \pm 0.42 \pm 0.58$ & 0.220 & 82.4 & $11.29 \pm 0.34 \pm 0.48$ \\
\hline $0.24-0.30$ & 0.269 & 67.0 & $13.86 \pm 0.31 \pm 0.42$ & 0.268 & 82.0 & $8.66 \pm 0.24 \pm 0.29$ \\
\hline $0.30-0.36$ & 0.329 & 66.9 & $10.08 \pm 0.26 \pm 0.31$ & 0.328 & 81.8 & $5.91 \pm 0.20 \pm 0.24$ \\
\hline $0.36-0.42$ & 0.388 & 66.8 & $8.08 \pm 0.23 \pm 0.32$ & 0.388 & 82.0 & $4.51 \pm 0.17 \pm 0.20$ \\
\hline $0.42-0.50$ & 0.458 & 66.7 & $5.41 \pm 0.16 \pm 0.25$ & 0.456 & 81.6 & $3.29 \pm 0.13 \pm 0.18$ \\
\hline $0.50-0.60$ & 0.546 & 66.7 & $3.23 \pm 0.11 \pm 0.19$ & 0.543 & 81.3 & $1.79 \pm 0.08 \pm 0.13$ \\
\hline $0.60-0.72$ & 0.654 & 66.2 & $1.60 \pm 0.07 \pm 0.12$ & 0.652 & 81.2 & $0.88 \pm 0.06 \pm 0.08$ \\
\hline $0.72-0.90$ & 0.790 & 66.4 & $0.74 \pm 0.04 \pm 0.08$ & 0.786 & 81.0 & $0.30 \pm 0.02 \pm 0.04$ \\
\hline $0.90-1.25$ & 1.017 & 66.2 & $0.12 \pm 0.01 \pm 0.02$ & 1.004 & 81.4 & $0.04 \pm 0.01 \pm 0.01$ \\
\hline \multirow[t]{2}{*}{$p_{\mathrm{T}}$} & \multicolumn{3}{|c|}{$90<\theta<105$} & \multicolumn{3}{|c|}{$105<\theta<125$} \\
\hline & $\left\langle p_{\mathrm{T}}\right\rangle$ & $\langle\theta\rangle$ & $\mathrm{d}^{2} \sigma / \mathrm{d} p \mathrm{~d} \Omega$ & $\overline{\left\langle p_{\mathrm{T}}\right\rangle}$ & $\langle\theta\rangle$ & $\mathrm{d}^{2} \sigma / \mathrm{d} p \mathrm{~d} \Omega$ \\
\hline $0.13-0.16$ & 0.145 & 97.3 & $10.63 \pm 0.38 \pm 0.77$ & 0.145 & 114.9 & $8.61 \pm 0.28 \pm 0.72$ \\
\hline $0.16-0.20$ & 0.179 & 97.1 & $10.19 \pm 0.31 \pm 0.63$ & 0.179 & 114.0 & $6.45 \pm 0.20 \pm 0.44$ \\
\hline $0.20-0.24$ & 0.219 & 97.0 & $7.74 \pm 0.26 \pm 0.43$ & 0.218 & 113.6 & $5.65 \pm 0.20 \pm 0.28$ \\
\hline $0.24-0.30$ & 0.267 & 97.0 & $6.15 \pm 0.20 \pm 0.27$ & 0.267 & 113.8 & $3.38 \pm 0.13 \pm 0.16$ \\
\hline $0.30-0.36$ & 0.328 & 97.0 & $4.37 \pm 0.17 \pm 0.19$ & 0.326 & 113.3 & $2.20 \pm 0.11 \pm 0.13$ \\
\hline $0.36-0.42$ & 0.388 & 96.7 & $2.61 \pm 0.13 \pm 0.16$ & 0.390 & 112.9 & $1.27 \pm 0.08 \pm 0.10$ \\
\hline $0.42-0.50$ & 0.457 & 96.8 & $1.65 \pm 0.09 \pm 0.12$ & 0.454 & 113.6 & $0.74 \pm 0.05 \pm 0.07$ \\
\hline $0.50-0.60$ & 0.543 & 96.7 & $0.89 \pm 0.06 \pm 0.09$ & 0.540 & 111.9 & $0.24 \pm 0.03 \pm 0.03$ \\
\hline $0.60-0.72$ & 0.649 & 95.8 & $0.38 \pm 0.04 \pm 0.05$ & 0.656 & 111.2 & $0.09 \pm 0.02 \pm 0.02$ \\
\hline $0.72-0.90$ & 0.791 & 96.2 & $0.07 \pm 0.02 \pm 0.02$ & & & \\
\hline
\end{tabular}


Table 8 Double-differential inclusive cross-section $\mathrm{d}^{2} \sigma / \mathrm{d} p \mathrm{~d} \Omega[\mathrm{mb} /(\mathrm{GeV} / c \mathrm{sr})]$ of the production of protons in $\pi^{-}+\mathrm{Be} \rightarrow \mathrm{p}+\mathrm{X}$ interactions with $-8.0 \mathrm{GeV} / c$ beam momentum; the first error is statistical, the second systematic; $p_{\mathrm{T}}$ in $\mathrm{GeV} / c$, polar angle $\theta$ in degrees

\begin{tabular}{|c|c|c|c|c|c|c|}
\hline \multirow[t]{2}{*}{$p_{\mathrm{T}}$} & \multicolumn{3}{|c|}{$20<\theta<30$} & \multicolumn{3}{|c|}{$30<\theta<40$} \\
\hline & $\overline{\left\langle p_{\mathrm{T}}\right\rangle}$ & $\langle\theta\rangle$ & $\mathrm{d}^{2} \sigma / \mathrm{d} p \mathrm{~d} \Omega$ & $\overline{\left\langle p_{\mathrm{T}}\right\rangle}$ & $\langle\theta\rangle$ & $\mathrm{d}^{2} \sigma / \mathrm{d} p \mathrm{~d} \Omega$ \\
\hline $0.20-0.24$ & 0.221 & 25.1 & $28.46 \pm 1.02 \pm 1.39$ & & & \\
\hline $0.24-0.30$ & 0.269 & 25.0 & $26.71 \pm 0.79 \pm 1.15$ & 0.271 & 35.0 & $26.35 \pm 0.77 \pm 1.07$ \\
\hline $0.30-0.36$ & 0.329 & 24.9 & $27.13 \pm 0.79 \pm 1.06$ & 0.330 & 34.9 & $25.06 \pm 0.75 \pm 0.93$ \\
\hline $0.36-0.42$ & 0.389 & 25.0 & $24.92 \pm 0.76 \pm 0.89$ & 0.389 & 35.0 & $23.28 \pm 0.73 \pm 0.79$ \\
\hline $0.42-0.50$ & 0.459 & 25.0 & $22.28 \pm 0.60 \pm 0.73$ & 0.459 & 35.0 & $17.73 \pm 0.55 \pm 0.57$ \\
\hline $0.50-0.60$ & 0.547 & 24.9 & $19.55 \pm 0.50 \pm 0.65$ & 0.548 & 34.8 & $16.55 \pm 0.48 \pm 0.54$ \\
\hline $0.60-0.72$ & 0.658 & 24.9 & $14.55 \pm 0.40 \pm 0.57$ & 0.656 & 34.8 & $12.50 \pm 0.38 \pm 0.49$ \\
\hline $0.72-0.90$ & & & & 0.801 & 34.9 & $7.74 \pm 0.24 \pm 0.40$ \\
\hline \multirow[t]{2}{*}{$p_{\mathrm{T}}$} & \multicolumn{3}{|c|}{$40<\theta<50$} & \multicolumn{3}{|c|}{$50<\theta<60$} \\
\hline & $\left\langle p_{\mathrm{T}}\right\rangle$ & $\langle\theta\rangle$ & $\mathrm{d}^{2} \sigma / \mathrm{d} p \mathrm{~d} \Omega$ & $\left\langle p_{\mathrm{T}}\right\rangle$ & $\langle\theta\rangle$ & $\mathrm{d}^{2} \sigma / \mathrm{d} p \mathrm{~d} \Omega$ \\
\hline $0.30-0.36$ & 0.329 & 45.0 & $24.35 \pm 0.73 \pm 0.82$ & & & \\
\hline $0.36-0.42$ & 0.389 & 44.9 & $20.62 \pm 0.67 \pm 0.63$ & 0.389 & 54.9 & $20.67 \pm 0.67 \pm 0.65$ \\
\hline $0.42-0.50$ & 0.457 & 45.0 & $18.61 \pm 0.56 \pm 0.57$ & 0.458 & 55.0 & $16.70 \pm 0.52 \pm 0.53$ \\
\hline $0.50-0.60$ & 0.547 & 44.8 & $14.12 \pm 0.45 \pm 0.47$ & 0.548 & 55.0 & $12.55 \pm 0.43 \pm 0.46$ \\
\hline $0.60-0.72$ & 0.656 & 44.9 & $9.24 \pm 0.33 \pm 0.37$ & 0.655 & 54.9 & $8.68 \pm 0.33 \pm 0.38$ \\
\hline $0.72-0.90$ & 0.799 & 45.0 & $6.23 \pm 0.23 \pm 0.34$ & 0.800 & 55.0 & $4.79 \pm 0.20 \pm 0.28$ \\
\hline $0.90-1.25$ & 1.046 & 44.9 & $1.78 \pm 0.09 \pm 0.15$ & 1.044 & 54.9 & $1.37 \pm 0.08 \pm 0.13$ \\
\hline \multirow[t]{2}{*}{$p_{\mathrm{T}}$} & \multicolumn{3}{|c|}{$60<\theta<75$} & \multicolumn{3}{|c|}{$75<\theta<90$} \\
\hline & $\overline{\left\langle p_{\mathrm{T}}\right\rangle}$ & $\langle\theta\rangle$ & $\mathrm{d}^{2} \sigma / \mathrm{d} p \mathrm{~d} \Omega$ & $\overline{\left\langle p_{\mathrm{T}}\right\rangle}$ & $\langle\theta\rangle$ & $\mathrm{d}^{2} \sigma / \mathrm{d} p \mathrm{~d} \Omega$ \\
\hline $0.36-0.42$ & 0.389 & 67.5 & $18.23 \pm 0.50 \pm 0.61$ & & & \\
\hline $0.42-0.50$ & 0.458 & 67.4 & $16.13 \pm 0.42 \pm 0.47$ & 0.458 & 81.7 & $10.95 \pm 0.34 \pm 0.42$ \\
\hline $0.50-0.60$ & 0.546 & 67.4 & $11.55 \pm 0.33 \pm 0.43$ & 0.546 & 81.7 & $7.54 \pm 0.26 \pm 0.34$ \\
\hline $0.60-0.72$ & 0.654 & 67.2 & $6.82 \pm 0.24 \pm 0.36$ & 0.655 & 81.7 & $3.88 \pm 0.18 \pm 0.27$ \\
\hline $0.72-0.90$ & 0.797 & 67.2 & $3.50 \pm 0.15 \pm 0.27$ & 0.796 & 81.7 & $1.69 \pm 0.10 \pm 0.15$ \\
\hline $0.90-1.25$ & 1.038 & 66.7 & $0.78 \pm 0.05 \pm 0.10$ & 1.024 & 81.7 & $0.44 \pm 0.04 \pm 0.06$ \\
\hline \multirow[t]{2}{*}{$p_{\mathrm{T}}$} & \multicolumn{3}{|c|}{$90<\theta<105$} & \multicolumn{3}{|c|}{$105<\theta<125$} \\
\hline & $\overline{\left\langle p_{\mathrm{T}}\right\rangle}$ & $\langle\theta\rangle$ & $\mathrm{d}^{2} \sigma / \mathrm{d} p \mathrm{~d} \Omega$ & $\overline{\left\langle p_{\mathrm{T}}\right\rangle}$ & $\langle\theta\rangle$ & $\mathrm{d}^{2} \sigma / \mathrm{d} p \mathrm{~d} \Omega$ \\
\hline $0.36-0.42$ & & & & 0.388 & 113.6 & $4.91 \pm 0.23 \pm 0.19$ \\
\hline $0.42-0.50$ & 0.457 & 96.6 & $6.78 \pm 0.27 \pm 0.34$ & 0.456 & 113.6 & $3.32 \pm 0.16 \pm 0.16$ \\
\hline $0.50-0.60$ & 0.545 & 96.7 & $4.23 \pm 0.20 \pm 0.26$ & 0.541 & 113.0 & $1.88 \pm 0.12 \pm 0.15$ \\
\hline $0.60-0.72$ & 0.657 & 96.5 & $2.04 \pm 0.14 \pm 0.17$ & 0.654 & 113.2 & $0.79 \pm 0.07 \pm 0.10$ \\
\hline $0.72-0.90$ & 0.795 & 96.6 & $0.79 \pm 0.07 \pm 0.08$ & 0.792 & 111.9 & $0.29 \pm 0.04 \pm 0.05$ \\
\hline $0.90-1.25$ & 1.018 & 96.1 & $0.23 \pm 0.03 \pm 0.03$ & 1.035 & 111.8 & $0.05 \pm 0.02 \pm 0.02$ \\
\hline
\end{tabular}

which were obtained with somewhat higher beam momenta. A more direct comparison with these data will be given in a forthcoming paper [22] where we present cross-sections on Be for all beam momenta from $3 \mathrm{GeV} / c$ to $15 \mathrm{GeV} / c$.

\subsection{Comparison with E802 results}

Experiment E802 [23] at Brookhaven National Laboratory measured secondary $\pi^{+}$'s in the polar-angle range $5^{\circ}<\theta<$ $58^{\circ}$ from the interactions of $14.6 \mathrm{GeV} / c$ protons with beryllium nuclei.

Figure 16 shows their published Lorentz-invariant crosssection of $\pi^{+}$and $\pi^{-}$production by $14.6 \mathrm{GeV} / c$ protons, in the rapidity range $1.2<y<1.4$, as a function of $m_{\mathrm{T}}-$ $m_{\pi}$, where $m_{\mathrm{T}}$ denotes the transverse mass. Their data are compared with our results expressed in the same units as used by E802. 
Table 9 Double-differential inclusive cross-section $\mathrm{d}^{2} \sigma / \mathrm{d} p \mathrm{~d} \Omega[\mathrm{mb} /(\mathrm{GeV} / \mathrm{csr})]$ of the production of $\pi^{+}$'s in $\pi^{-}+\mathrm{Be} \rightarrow \pi^{+}+\mathrm{X}$ interactions with $-8.0 \mathrm{GeV} / c$ beam momentum; the first error is statistical, the second systematic; $p_{\mathrm{T}}$ in $\mathrm{GeV} / c$, polar angle $\theta$ in degrees

\begin{tabular}{|c|c|c|c|c|c|c|}
\hline \multirow[t]{2}{*}{$p_{\mathrm{T}}$} & \multicolumn{3}{|c|}{$20<\theta<30$} & \multicolumn{3}{|c|}{$30<\theta<40$} \\
\hline & $\left\langle p_{\mathrm{T}}\right\rangle$ & $\langle\theta\rangle$ & $\mathrm{d}^{2} \sigma / \mathrm{d} p \mathrm{~d} \Omega$ & $\left\langle p_{\mathrm{T}}\right\rangle$ & $\langle\theta\rangle$ & $\mathrm{d}^{2} \sigma / \mathrm{d} p \mathrm{~d} \Omega$ \\
\hline $0.10-0.13$ & 0.116 & 24.5 & $40.03 \pm 1.60 \pm 2.82$ & 0.116 & 34.7 & $27.55 \pm 1.32 \pm 1.90$ \\
\hline $0.13-0.16$ & 0.146 & 24.6 & $51.88 \pm 1.74 \pm 2.87$ & 0.145 & 34.8 & $38.40 \pm 1.48 \pm 2.07$ \\
\hline $0.16-0.20$ & 0.181 & 24.5 & $58.09 \pm 1.48 \pm 2.61$ & 0.181 & 34.8 & $40.43 \pm 1.25 \pm 1.79$ \\
\hline $0.20-0.24$ & 0.220 & 24.6 & $61.57 \pm 1.52 \pm 2.38$ & 0.220 & 34.6 & $41.25 \pm 1.23 \pm 1.59$ \\
\hline $0.24-0.30$ & 0.270 & 24.7 & $58.68 \pm 1.19 \pm 1.94$ & 0.269 & 34.7 & $43.31 \pm 1.02 \pm 1.42$ \\
\hline $0.30-0.36$ & 0.328 & 24.6 & $53.20 \pm 1.14 \pm 1.58$ & 0.329 & 34.8 & $36.40 \pm 0.94 \pm 1.07$ \\
\hline $0.36-0.42$ & 0.389 & 24.6 & $41.85 \pm 0.99 \pm 1.35$ & 0.389 & 34.7 & $28.53 \pm 0.82 \pm 0.83$ \\
\hline $0.42-0.50$ & 0.457 & 24.6 & $31.72 \pm 0.74 \pm 1.11$ & 0.457 & 34.6 & $19.60 \pm 0.58 \pm 0.63$ \\
\hline $0.50-0.60$ & 0.545 & 24.7 & $21.39 \pm 0.53 \pm 1.04$ & 0.545 & 34.8 & $12.58 \pm 0.40 \pm 0.55$ \\
\hline $0.60-0.72$ & 0.655 & 24.7 & $11.72 \pm 0.34 \pm 0.84$ & 0.656 & 34.7 & $7.16 \pm 0.26 \pm 0.46$ \\
\hline $0.72-0.90$ & & & & 0.796 & 34.5 & $2.87 \pm 0.12 \pm 0.30$ \\
\hline \multirow[t]{2}{*}{$p_{\mathrm{T}}$} & \multicolumn{3}{|c|}{$40<\theta<50$} & \multicolumn{3}{|c|}{$50<\theta<60$} \\
\hline & $\overline{\left\langle p_{\mathrm{T}}\right\rangle}$ & $\langle\theta\rangle$ & $\mathrm{d}^{2} \sigma / \mathrm{d} p \mathrm{~d} \Omega$ & $\left\langle p_{\mathrm{T}}\right\rangle$ & $\langle\theta\rangle$ & $\mathrm{d}^{2} \sigma / \mathrm{d} p \mathrm{~d} \Omega$ \\
\hline $0.10-0.13$ & 0.117 & 45.0 & $21.40 \pm 1.17 \pm 1.54$ & & & \\
\hline $0.13-0.16$ & 0.146 & 44.9 & $25.24 \pm 1.15 \pm 1.38$ & 0.146 & 54.9 & $19.22 \pm 0.99 \pm 1.14$ \\
\hline $0.16-0.20$ & 0.179 & 44.8 & $29.56 \pm 1.07 \pm 1.34$ & 0.180 & 54.8 & $21.66 \pm 0.90 \pm 0.99$ \\
\hline $0.20-0.24$ & 0.220 & 44.9 & $29.03 \pm 1.03 \pm 1.14$ & 0.220 & 54.7 & $22.20 \pm 0.92 \pm 0.87$ \\
\hline $0.24-0.30$ & 0.270 & 44.7 & $26.44 \pm 0.80 \pm 0.88$ & 0.269 & 54.9 & $20.48 \pm 0.71 \pm 0.68$ \\
\hline $0.30-0.36$ & 0.329 & 44.7 & $23.88 \pm 0.76 \pm 0.72$ & 0.329 & 54.7 & $16.76 \pm 0.62 \pm 0.51$ \\
\hline $0.36-0.42$ & 0.388 & 44.7 & $18.64 \pm 0.66 \pm 0.56$ & 0.389 & 54.8 & $14.55 \pm 0.59 \pm 0.47$ \\
\hline $0.42-0.50$ & 0.457 & 44.7 & $13.14 \pm 0.48 \pm 0.45$ & 0.458 & 54.6 & $8.92 \pm 0.38 \pm 0.32$ \\
\hline $0.50-0.60$ & 0.544 & 44.5 & $8.67 \pm 0.33 \pm 0.37$ & 0.544 & 54.5 & $5.77 \pm 0.27 \pm 0.34$ \\
\hline $0.60-0.72$ & 0.656 & 44.6 & $4.57 \pm 0.21 \pm 0.29$ & 0.653 & 54.4 & $3.28 \pm 0.18 \pm 0.22$ \\
\hline $0.72-0.90$ & 0.789 & 44.9 & $1.66 \pm 0.09 \pm 0.16$ & 0.790 & 54.6 & $1.38 \pm 0.09 \pm 0.14$ \\
\hline $0.90-1.25$ & & & & 1.016 & 54.2 & $0.22 \pm 0.02 \pm 0.03$ \\
\hline \multirow[t]{2}{*}{$p_{\mathrm{T}}$} & \multicolumn{3}{|c|}{$60<\theta<75$} & \multicolumn{3}{|c|}{$75<\theta<90$} \\
\hline & $\left\langle p_{\mathrm{T}}\right\rangle$ & $\langle\theta\rangle$ & $\mathrm{d}^{2} \sigma / \mathrm{d} p \mathrm{~d} \Omega$ & $\left\langle p_{\mathrm{T}}\right\rangle$ & $\langle\theta\rangle$ & $\mathrm{d}^{2} \sigma / \mathrm{d} p \mathrm{~d} \Omega$ \\
\hline $0.13-0.16$ & 0.146 & 67.2 & $13.44 \pm 0.66 \pm 0.80$ & 0.145 & 82.6 & $11.17 \pm 0.62 \pm 0.74$ \\
\hline $0.16-0.20$ & 0.180 & 67.2 & $16.66 \pm 0.63 \pm 0.82$ & 0.180 & 82.2 & $12.06 \pm 0.52 \pm 0.58$ \\
\hline $0.20-0.24$ & 0.220 & 66.9 & $15.91 \pm 0.63 \pm 0.61$ & 0.219 & 82.3 & $11.14 \pm 0.50 \pm 0.46$ \\
\hline $0.24-0.30$ & 0.269 & 67.2 & $13.82 \pm 0.47 \pm 0.45$ & 0.269 & 82.0 & $8.48 \pm 0.35 \pm 0.28$ \\
\hline $0.30-0.36$ & 0.329 & 67.2 & $10.10 \pm 0.39 \pm 0.31$ & 0.330 & 81.6 & $6.09 \pm 0.30 \pm 0.22$ \\
\hline $0.36-0.42$ & 0.390 & 66.7 & $7.17 \pm 0.32 \pm 0.26$ & 0.389 & 82.0 & $4.01 \pm 0.24 \pm 0.18$ \\
\hline $0.42-0.50$ & 0.457 & 66.8 & $5.45 \pm 0.24 \pm 0.23$ & 0.457 & 81.5 & $3.30 \pm 0.19 \pm 0.17$ \\
\hline $0.50-0.60$ & 0.545 & 66.5 & $3.04 \pm 0.15 \pm 0.17$ & 0.544 & 81.7 & $1.70 \pm 0.12 \pm 0.12$ \\
\hline $0.60-0.72$ & 0.653 & 66.9 & $1.82 \pm 0.11 \pm 0.14$ & 0.655 & 81.4 & $0.95 \pm 0.09 \pm 0.08$ \\
\hline $0.72-0.90$ & 0.785 & 66.5 & $0.65 \pm 0.05 \pm 0.07$ & 0.791 & 81.5 & $0.28 \pm 0.03 \pm 0.04$ \\
\hline $0.90-1.25$ & 1.018 & 65.1 & $0.09 \pm 0.01 \pm 0.02$ & 1.006 & 81.2 & $0.04 \pm 0.01 \pm 0.02$ \\
\hline \multirow[t]{2}{*}{$p_{\mathrm{T}}$} & \multicolumn{3}{|c|}{$90<\theta<105$} & \multicolumn{3}{|c|}{$105<\theta<125$} \\
\hline & $\left\langle p_{\mathrm{T}}\right\rangle$ & $\langle\theta\rangle$ & $\mathrm{d}^{2} \sigma / \mathrm{d} p \mathrm{~d} \Omega$ & $\overline{\left\langle p_{\mathrm{T}}\right\rangle}$ & $\langle\theta\rangle$ & $\mathrm{d}^{2} \sigma / \mathrm{d} p \mathrm{~d} \Omega$ \\
\hline $0.13-0.16$ & 0.146 & 97.4 & $9.44 \pm 0.55 \pm 0.66$ & 0.145 & 114.3 & $7.67 \pm 0.41 \pm 0.55$ \\
\hline $0.16-0.20$ & 0.180 & 97.2 & $9.09 \pm 0.45 \pm 0.52$ & 0.179 & 114.2 & $6.20 \pm 0.31 \pm 0.38$ \\
\hline $0.20-0.24$ & 0.219 & 97.4 & $7.08 \pm 0.37 \pm 0.31$ & 0.218 & 114.0 & $5.35 \pm 0.29 \pm 0.27$ \\
\hline $0.24-0.30$ & 0.269 & 97.1 & $5.74 \pm 0.29 \pm 0.26$ & 0.269 & 113.6 & $3.14 \pm 0.18 \pm 0.14$ \\
\hline $0.30-0.36$ & 0.328 & 96.9 & $3.62 \pm 0.23 \pm 0.16$ & 0.327 & 113.6 & $2.08 \pm 0.15 \pm 0.11$ \\
\hline $0.36-0.42$ & 0.388 & 96.6 & $2.64 \pm 0.19 \pm 0.15$ & 0.387 & 112.0 & $1.27 \pm 0.12 \pm 0.10$ \\
\hline $0.42-0.50$ & 0.459 & 97.1 & $1.84 \pm 0.14 \pm 0.14$ & 0.456 & 113.1 & $0.69 \pm 0.07 \pm 0.06$ \\
\hline $0.50-0.60$ & 0.544 & 96.9 & $0.86 \pm 0.09 \pm 0.08$ & 0.536 & 113.0 & $0.45 \pm 0.06 \pm 0.06$ \\
\hline $0.60-0.72$ & 0.649 & 95.8 & $0.58 \pm 0.07 \pm 0.07$ & 0.652 & 111.7 & $0.10 \pm 0.02 \pm 0.02$ \\
\hline $0.72-0.90$ & 0.796 & 96.9 & $0.13 \pm 0.02 \pm 0.02$ & 0.768 & 112.2 & $0.05 \pm 0.02 \pm 0.02$ \\
\hline
\end{tabular}


Table 10 Double-differential inclusive cross-section $\mathrm{d}^{2} \sigma / \mathrm{d} p \mathrm{~d} \Omega[\mathrm{mb} /(\mathrm{GeV} / c \mathrm{sr})]$ of the production of $\pi^{-}$'s in $\pi^{-}+\mathrm{Be} \rightarrow \pi^{-}+\mathrm{X}$ interactions with $-8.0 \mathrm{GeV} / c$ beam momentum; the first error is statistical, the second systematic; $p_{\mathrm{T}}$ in $\mathrm{GeV} / c$, polar angle $\theta$ in degrees

\begin{tabular}{|c|c|c|c|c|c|c|}
\hline \multirow[t]{2}{*}{$p_{\mathrm{T}}$} & \multicolumn{3}{|c|}{$20<\theta<30$} & \multicolumn{3}{|c|}{$30<\theta<40$} \\
\hline & $\left\langle p_{\mathrm{T}}\right\rangle$ & $\langle\theta\rangle$ & $\mathrm{d}^{2} \sigma / \mathrm{d} p \mathrm{~d} \Omega$ & $\left\langle p_{\mathrm{T}}\right\rangle$ & $\langle\theta\rangle$ & $\mathrm{d}^{2} \sigma / \mathrm{d} p \mathrm{~d} \Omega$ \\
\hline $0.10-0.13$ & 0.116 & 24.8 & $62.88 \pm 2.02 \pm 4.24$ & 0.116 & 34.8 & $41.80 \pm 1.61 \pm 2.90$ \\
\hline $0.13-0.16$ & 0.145 & 24.6 & $85.27 \pm 2.21 \pm 4.78$ & 0.145 & 34.5 & $53.90 \pm 1.73 \pm 2.88$ \\
\hline $0.16-0.20$ & 0.180 & 24.6 & $95.38 \pm 1.93 \pm 4.18$ & 0.181 & 34.7 & $63.73 \pm 1.57 \pm 2.83$ \\
\hline $0.20-0.24$ & 0.220 & 24.7 & $97.33 \pm 1.93 \pm 3.65$ & 0.220 & 34.7 & $67.33 \pm 1.59 \pm 2.57$ \\
\hline $0.24-0.30$ & 0.269 & 24.7 & $96.82 \pm 1.56 \pm 3.05$ & 0.269 & 34.6 & $62.27 \pm 1.23 \pm 1.98$ \\
\hline $0.30-0.36$ & 0.329 & 24.8 & $83.57 \pm 1.45 \pm 2.36$ & 0.329 & 34.5 & $52.83 \pm 1.12 \pm 1.49$ \\
\hline $0.36-0.42$ & 0.389 & 24.8 & $67.94 \pm 1.30 \pm 2.05$ & 0.388 & 34.7 & $43.38 \pm 1.03 \pm 1.23$ \\
\hline $0.42-0.50$ & 0.458 & 24.7 & $55.32 \pm 1.01 \pm 1.86$ & 0.458 & 34.7 & $34.45 \pm 0.79 \pm 1.12$ \\
\hline $0.50-0.60$ & 0.547 & 24.5 & $34.34 \pm 0.71 \pm 1.53$ & 0.545 & 34.6 & $23.14 \pm 0.58 \pm 0.98$ \\
\hline $0.60-0.72$ & 0.654 & 24.4 & $21.28 \pm 0.51 \pm 1.26$ & 0.655 & 34.8 & $12.91 \pm 0.39 \pm 0.75$ \\
\hline $0.72-0.90$ & & & & 0.799 & 34.9 & $5.46 \pm 0.20 \pm 0.44$ \\
\hline \multirow[t]{2}{*}{$p_{\mathrm{T}}$} & \multicolumn{3}{|c|}{$40<\theta<50$} & \multicolumn{3}{|c|}{$50<\theta<60$} \\
\hline & $\overline{\left\langle p_{\mathrm{T}}\right\rangle}$ & $\langle\theta\rangle$ & $\mathrm{d}^{2} \sigma / \mathrm{d} p \mathrm{~d} \Omega$ & $\left\langle p_{\mathrm{T}}\right\rangle$ & $\langle\theta\rangle$ & $\mathrm{d}^{2} \sigma / \mathrm{d} p \mathrm{~d} \Omega$ \\
\hline $0.10-0.13$ & 0.115 & 44.9 & $30.80 \pm 1.33 \pm 2.28$ & & & \\
\hline $0.13-0.16$ & 0.146 & 44.9 & $39.52 \pm 1.44 \pm 2.16$ & 0.145 & 54.8 & $28.11 \pm 1.18 \pm 1.73$ \\
\hline $0.16-0.20$ & 0.180 & 44.9 & $46.83 \pm 1.34 \pm 2.11$ & 0.180 & 54.8 & $33.93 \pm 1.13 \pm 1.54$ \\
\hline $0.20-0.24$ & 0.220 & 44.8 & $47.27 \pm 1.35 \pm 1.83$ & 0.220 & 54.8 & $31.77 \pm 1.08 \pm 1.21$ \\
\hline $0.24-0.30$ & 0.270 & 44.8 & $42.06 \pm 1.01 \pm 1.35$ & 0.269 & 54.6 & $30.99 \pm 0.87 \pm 0.99$ \\
\hline $0.30-0.36$ & 0.329 & 44.7 & $34.59 \pm 0.90 \pm 0.99$ & 0.329 & 54.7 & $25.41 \pm 0.78 \pm 0.75$ \\
\hline $0.36-0.42$ & 0.389 & 44.9 & $29.93 \pm 0.86 \pm 0.89$ & 0.389 & 54.6 & $19.27 \pm 0.67 \pm 0.60$ \\
\hline $0.42-0.50$ & 0.458 & 44.7 & $21.17 \pm 0.61 \pm 0.73$ & 0.458 & 54.7 & $14.23 \pm 0.50 \pm 0.52$ \\
\hline $0.50-0.60$ & 0.547 & 44.7 & $14.45 \pm 0.46 \pm 0.65$ & 0.546 & 54.6 & $9.78 \pm 0.37 \pm 0.47$ \\
\hline $0.60-0.72$ & 0.656 & 44.6 & $7.58 \pm 0.29 \pm 0.47$ & 0.654 & 54.6 & $5.35 \pm 0.24 \pm 0.34$ \\
\hline $0.72-0.90$ & 0.794 & 44.4 & $3.82 \pm 0.17 \pm 0.33$ & 0.793 & 54.7 & $2.41 \pm 0.14 \pm 0.21$ \\
\hline $0.90-1.25$ & & & & 1.023 & 54.2 & $0.41 \pm 0.04 \pm 0.06$ \\
\hline \multirow[t]{2}{*}{$p_{\mathrm{T}}$} & \multicolumn{3}{|c|}{$60<\theta<75$} & \multicolumn{3}{|c|}{$75<\theta<90$} \\
\hline & $\left\langle p_{\mathrm{T}}\right\rangle$ & $\langle\theta\rangle$ & $\mathrm{d}^{2} \sigma / \mathrm{d} p \mathrm{~d} \Omega$ & $\left\langle p_{\mathrm{T}}\right\rangle$ & $\langle\theta\rangle$ & $\mathrm{d}^{2} \sigma / \mathrm{d} p \mathrm{~d} \Omega$ \\
\hline $0.13-0.16$ & 0.146 & 67.3 & $22.50 \pm 0.85 \pm 1.35$ & 0.146 & 82.6 & $17.59 \pm 0.73 \pm 1.21$ \\
\hline $0.16-0.20$ & 0.180 & 67.3 & $26.16 \pm 0.78 \pm 1.20$ & 0.179 & 82.4 & $20.63 \pm 0.69 \pm 1.03$ \\
\hline $0.20-0.24$ & 0.219 & 67.0 & $23.97 \pm 0.75 \pm 0.87$ & 0.219 & 82.0 & $17.58 \pm 0.63 \pm 0.68$ \\
\hline $0.24-0.30$ & 0.268 & 67.0 & $21.19 \pm 0.58 \pm 0.65$ & 0.268 & 81.8 & $14.01 \pm 0.47 \pm 0.43$ \\
\hline $0.30-0.36$ & 0.329 & 66.8 & $16.07 \pm 0.49 \pm 0.48$ & 0.328 & 81.9 & $9.73 \pm 0.39 \pm 0.34$ \\
\hline $0.36-0.42$ & 0.389 & 67.0 & $12.21 \pm 0.44 \pm 0.41$ & 0.388 & 82.2 & $6.75 \pm 0.32 \pm 0.28$ \\
\hline $0.42-0.50$ & 0.456 & 66.8 & $8.85 \pm 0.32 \pm 0.36$ & 0.457 & 81.6 & $5.33 \pm 0.25 \pm 0.27$ \\
\hline $0.50-0.60$ & 0.545 & 66.9 & $5.73 \pm 0.23 \pm 0.31$ & 0.544 & 81.9 & $2.91 \pm 0.16 \pm 0.20$ \\
\hline $0.60-0.72$ & 0.655 & 66.8 & $2.66 \pm 0.14 \pm 0.19$ & 0.650 & 81.6 & $1.30 \pm 0.10 \pm 0.12$ \\
\hline $0.72-0.90$ & 0.793 & 66.4 & $1.17 \pm 0.08 \pm 0.12$ & 0.779 & 81.1 & $0.32 \pm 0.04 \pm 0.04$ \\
\hline $0.90-1.25$ & 1.006 & 66.4 & $0.10 \pm 0.02 \pm 0.02$ & 1.042 & 79.8 & $0.05 \pm 0.01 \pm 0.02$ \\
\hline \multirow[t]{2}{*}{$p_{\mathrm{T}}$} & \multicolumn{3}{|c|}{$90<\theta<105$} & \multicolumn{3}{|c|}{$105<\theta<125$} \\
\hline & $\left\langle p_{\mathrm{T}}\right\rangle$ & $\langle\theta\rangle$ & $\mathrm{d}^{2} \sigma / \mathrm{d} p \mathrm{~d} \Omega$ & $\overline{\left\langle p_{\mathrm{T}}\right\rangle}$ & $\langle\theta\rangle$ & $\mathrm{d}^{2} \sigma / \mathrm{d} p \mathrm{~d} \Omega$ \\
\hline $0.13-0.16$ & 0.145 & 97.2 & $15.89 \pm 0.69 \pm 1.21$ & 0.146 & 114.8 & $14.31 \pm 0.56 \pm 1.00$ \\
\hline $0.16-0.20$ & 0.180 & 97.4 & $16.27 \pm 0.58 \pm 0.89$ & 0.179 & 114.3 & $11.82 \pm 0.43 \pm 0.62$ \\
\hline $0.20-0.24$ & 0.219 & 97.3 & $13.44 \pm 0.54 \pm 0.57$ & 0.218 & 114.3 & $8.73 \pm 0.39 \pm 0.32$ \\
\hline $0.24-0.30$ & 0.267 & 96.8 & $9.30 \pm 0.38 \pm 0.31$ & 0.267 & 114.0 & $5.06 \pm 0.25 \pm 0.22$ \\
\hline $0.30-0.36$ & 0.327 & 97.0 & $6.47 \pm 0.32 \pm 0.27$ & 0.329 & 113.9 & $3.10 \pm 0.19 \pm 0.18$ \\
\hline $0.36-0.42$ & 0.389 & 96.9 & $4.37 \pm 0.26 \pm 0.24$ & 0.389 & 113.5 & $1.99 \pm 0.15 \pm 0.16$ \\
\hline $0.42-0.50$ & 0.456 & 96.4 & $2.87 \pm 0.19 \pm 0.21$ & 0.454 & 111.9 & $0.82 \pm 0.08 \pm 0.08$ \\
\hline $0.50-0.60$ & 0.545 & 95.9 & $1.38 \pm 0.11 \pm 0.14$ & 0.541 & 112.4 & $0.26 \pm 0.04 \pm 0.04$ \\
\hline $0.60-0.72$ & 0.655 & 96.3 & $0.41 \pm 0.05 \pm 0.06$ & 0.646 & 110.0 & $0.09 \pm 0.02 \pm 0.02$ \\
\hline $0.72-0.90$ & 0.790 & 96.8 & $0.08 \pm 0.02 \pm 0.02$ & & & \\
\hline
\end{tabular}




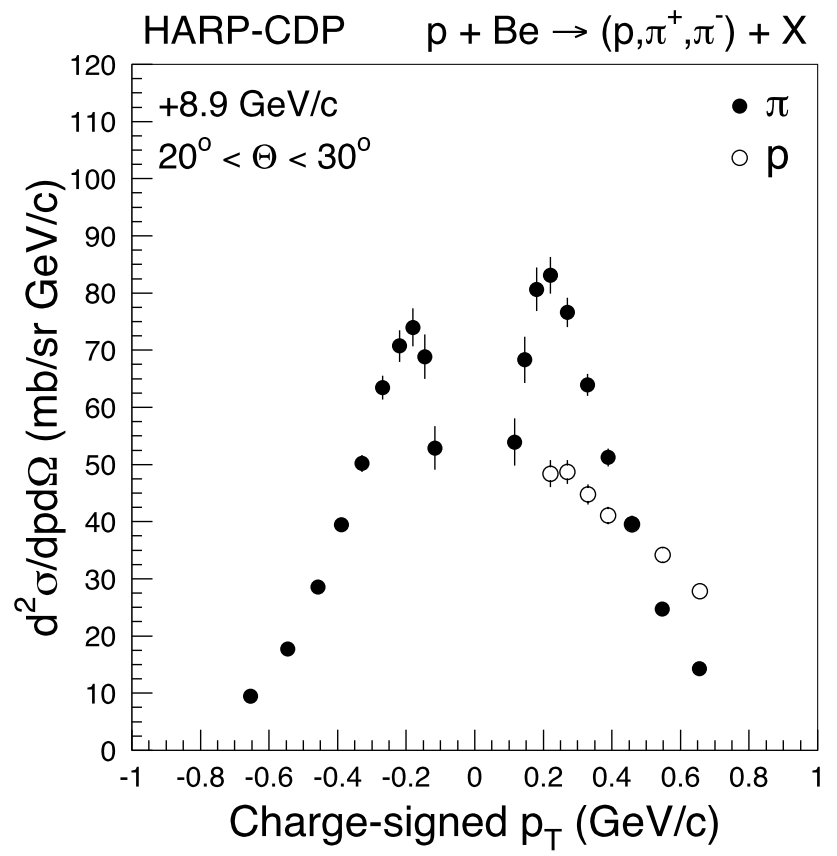

Fig. 13 Inclusive cross-sections as a function of charge-signed $p_{\mathrm{T}}$ of proton and $\pi^{ \pm}$production by $+8.9 \mathrm{GeV} / c$ incoming protons, off beryllium nuclei, in the polar-angle range $20^{\circ}<\theta<30^{\circ}$

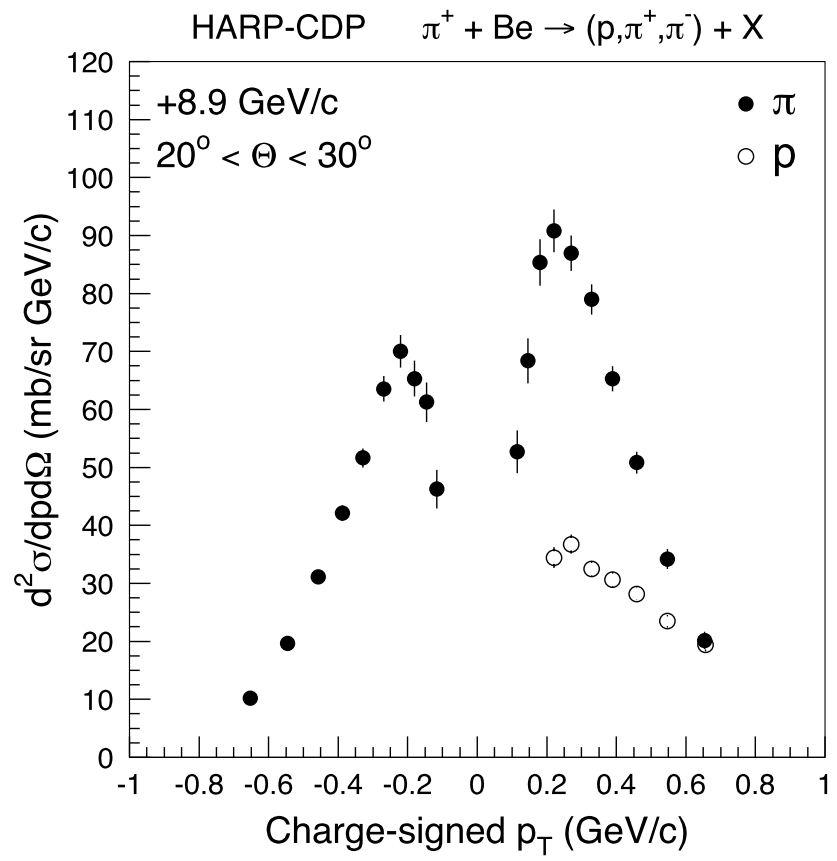

Fig. 14 Inclusive cross-sections as a function of charge-signed $p_{\mathrm{T}}$ of proton and $\pi^{ \pm}$production by $+8.9 \mathrm{GeV} / c$ incoming $\pi^{+}$'s, off beryllium nuclei, in the polar-angle range $20^{\circ}<\theta<30^{\circ}$

We note that both experiments agree in suggesting an exponential decrease of the invariant cross-section with increasing $m_{\mathrm{T}}-m_{\pi}$, over two orders of magnitude. Unlike the $\pi^{-}$cross-sections, the $\pi^{+}$cross-sections at +8.9 and

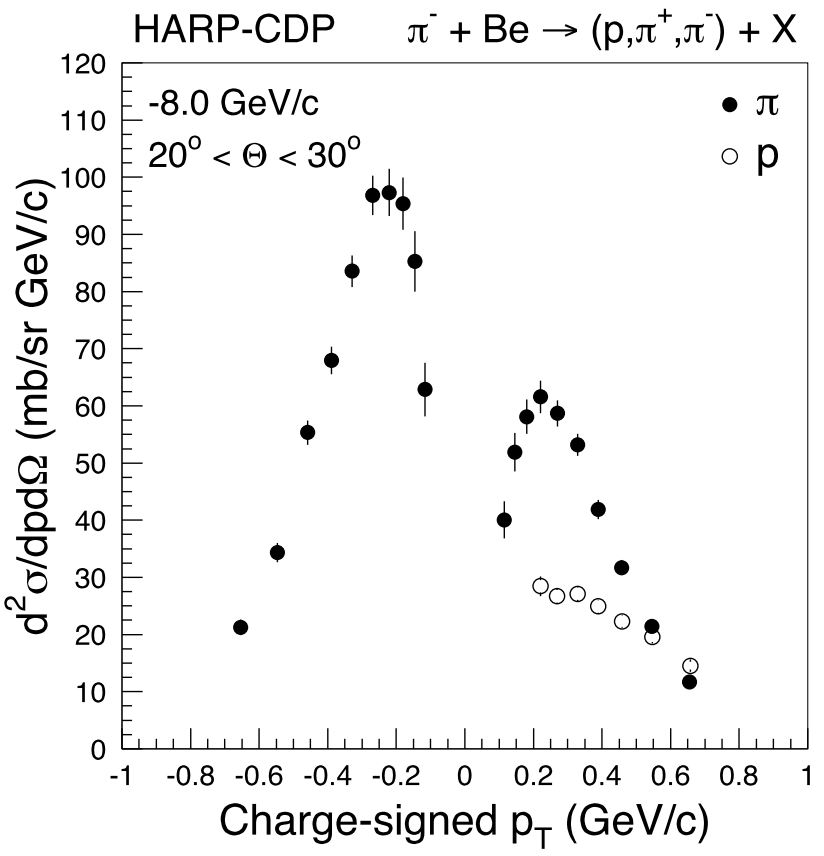

Fig. 15 Inclusive cross-sections as a function of charge-signed $p_{\mathrm{T}}$ of proton and $\pi^{ \pm}$production by $-8.0 \mathrm{GeV} / c$ incoming $\pi^{-}$'s, off beryllium nuclei, in the polar-angle range $20^{\circ}<\theta<30^{\circ}$

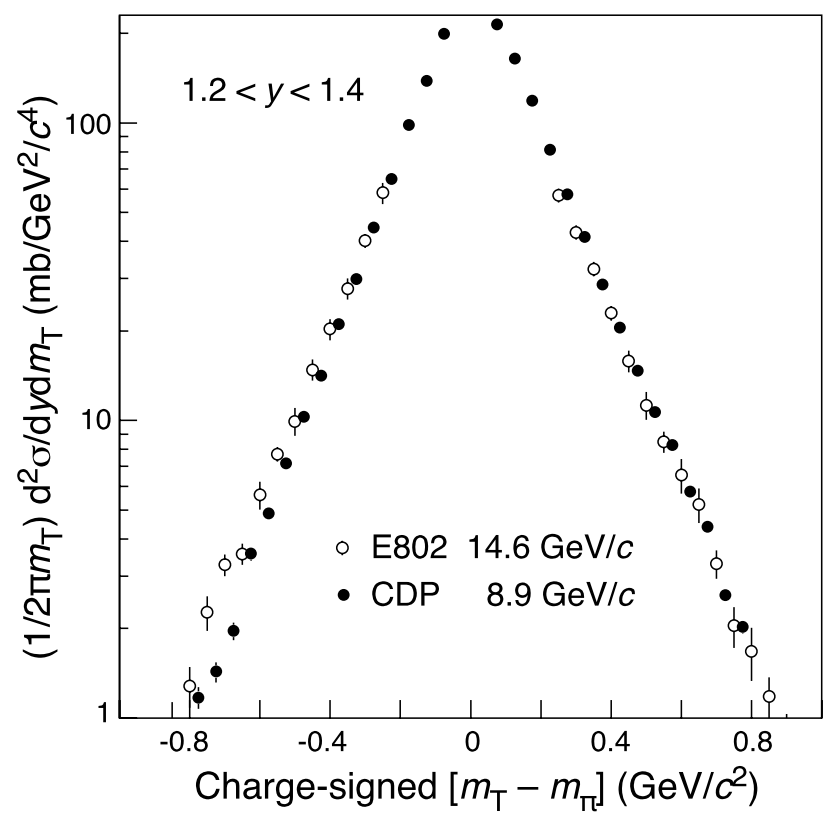

Fig. 16 Comparison of our cross-sections (black circles) of $\pi^{ \pm}$ production by $+8.9 \mathrm{GeV} / c$ protons off beryllium nuclei, with the cross-sections published by the E802 Collaboration for the proton beam momentum of $14.6 \mathrm{GeV} / c$ (open circles); the errors are statistical only

$+14.6 \mathrm{GeV} / c$ exhibit nearly the same slope. In the comparison of absolute cross-sections, the E802 normalization un- 


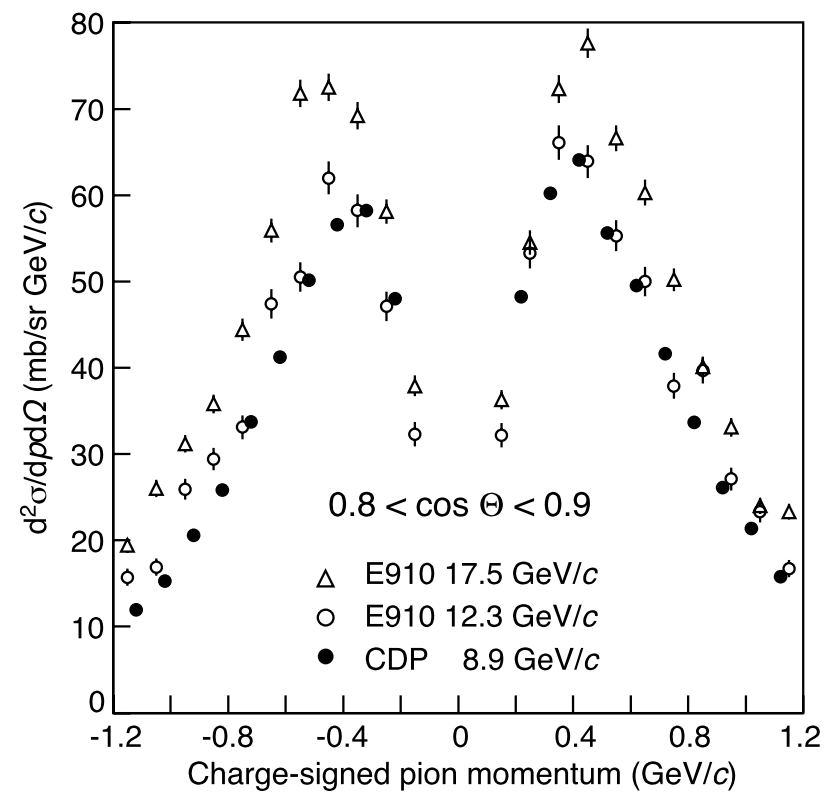

Fig. 17 Comparison of our cross-sections (black circles) of $\pi^{ \pm}$ production by $+8.9 \mathrm{GeV} / c$ protons off beryllium nuclei with the cross-sections published by the E910 Collaboration for proton beam momenta of 12.3 (open circles) and 17.5 (open triangles) $\mathrm{GeV} / c$; the errors are statistical only

certainty of (10-15)\% is to be taken into account on top of the beam energy difference.

\subsection{Comparison with E910 results}

Experiment E910 [24] at Brookhaven National Laboratory measured secondary charged pions in the momentum range $0.1-6 \mathrm{GeV} / c$ from the interactions of 12.3 and $17.5 \mathrm{GeV} / c$ protons with beryllium nuclei. This experiment used a TPC for the measurement of secondaries, with a comfortably large track length of $\sim 1.5 \mathrm{~m}$. With a magnetic field strength of $0.5 \mathrm{~T}$, this large track length renders charge identification and proton-pion separation by $\mathrm{d} E / \mathrm{d} x$ beyond doubt.

Also here, the E910 data are shown as published, and our data are expressed in the same units as used by E910. Although the E910 measurements were made with proton beam momenta of 12.3 and $17.5 \mathrm{GeV} / c$, respectively, we note the similar $\pi^{+} / \pi^{-}$ratio between the cross-sections from E910 and our cross-sections from a proton beam momentum of $8.9 \mathrm{GeV} / c$, shown in Fig. 17. In the comparison of absolute cross-sections, the E910 normalization uncertainty of $\leq 5 \%$ is to be taken into account on top of the beam energy differences.

\subsection{Comparison with results from the HARP Collaboration}

Figure 18 shows the comparison of our cross-sections of pion production by $+8.9 \mathrm{GeV} / c$ protons off beryllium nuclei with the results published by the HARP Collaboration [25],

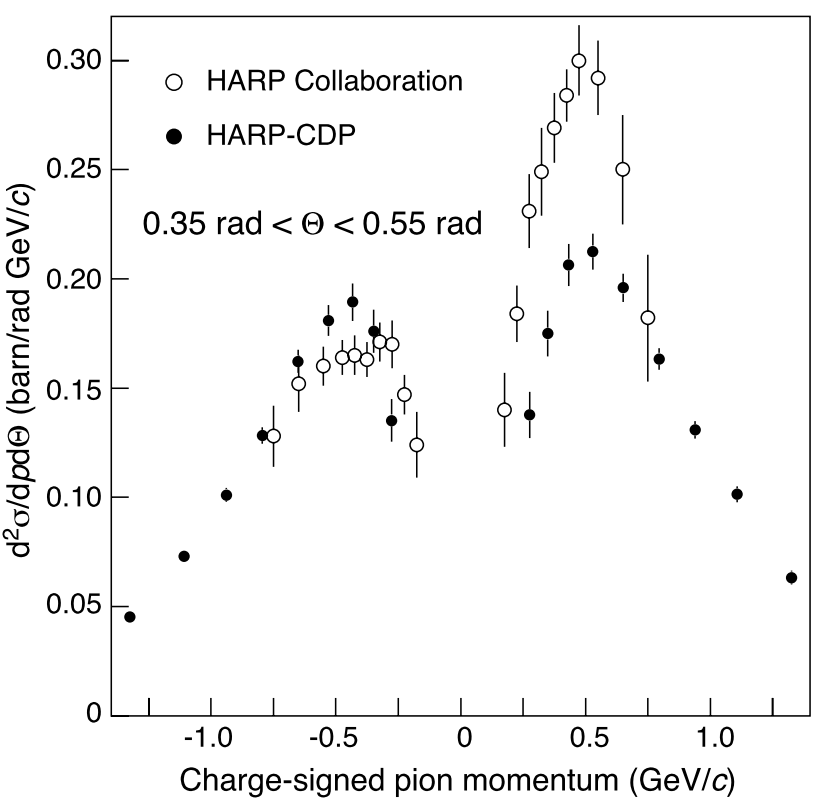

Fig. 18 Comparison of our cross-sections (black circles) of $\pi^{ \pm}$ production by $+8.9 \mathrm{GeV} / c$ protons off beryllium nuclei with the cross-sections published by the HARP Collaboration (open circles)

in the polar-angle range $0.35<\theta<0.55 \mathrm{rad}$. The latter cross-sections are plotted as published, while we expressed our cross-sections in the units used by the HARP Collaboration.

There is a severe discrepancy between our cross-sections and those reported by the HARP Collaboration. We note the difference especially of the $\pi^{+}$cross-section, and the difference in the momentum range. The discrepancy is even more serious as the same data set has been analysed by both groups. For a discussion of the reasons for this discrepancy we refer to the Appendix of this paper.

\section{Summary}

From the analysis of data from the HARP large-angle spectrometer (polar angle $\theta$ in the range $20^{\circ}<\theta<125^{\circ}$ ), double-differential cross-sections $\mathrm{d}^{2} \sigma / \mathrm{d} p \mathrm{~d} \Omega$ of the production of secondary protons, $\pi^{+}$'s, and $\pi^{-}$'s, have been obtained. The incoming beam particles were $+8.9 \mathrm{GeV} / c$ protons and pions, and $-8.0 \mathrm{GeV} / c$ pions, impinging on a $5 \%$ $\lambda_{\text {abs }}$ thick stationary beryllium target. The high statistics of the $+8.9 \mathrm{GeV} / c$ data allowed us to determine cross-sections of $\mathrm{K}^{+}$and deuteron production, albeit with lower precision. Our cross-sections for $\pi^{+}$and $\pi^{-}$production agree with results from other experiments but disagree with the results of the HARP Collaboration that were obtained from the same raw data.

Acknowledgements We are greatly indebted to many technical collaborators whose diligent and hard work made the HARP detector a 
well-functioning instrument. We thank all HARP colleagues who devoted time and effort to the design and construction of the detector, to data taking, and to setting up the computing and software infrastructure. We express our sincere gratitude to HARP's funding agencies for their support.

\section{Appendix}

The situation that two groups of authors, in this case the 'HARP Collaboration' (referred to as 'OH' for 'Official HARP') and us, the HARP-CDP group, publish inconsistent results from the same raw data, is unusual and unsatisfactory. Naturally, the question arises as to whose results can be trusted.

The central problem in OH's data analysis is their lack of understanding TPC track distortions which leads to:

- a bias of $\Delta\left(1 / p_{\mathrm{T}}\right) \simeq 0.3(\mathrm{GeV} / c)^{-1}$ in their reconstruction of TPC tracks; in other words, their relative $p_{\mathrm{T}}$ bias increases linearly with $p_{\mathrm{T}}$ and attains some $30 \%$ at $p_{\mathrm{T}}=$ $1 \mathrm{GeV} / c$; the bias is such that for particles with positive charge $p_{\mathrm{T}}$ is decreased, while for particles with negative charge $p_{\mathrm{T}}$ is increased;

- a resolution of $\sigma\left(1 / p_{\mathrm{T}}\right) \simeq 0.6(\mathrm{GeV} / c)^{-1}$ which is considerably worse than $\sigma\left(1 / p_{\mathrm{T}}\right) \simeq 0.30(\mathrm{GeV} / c)^{-1}$ claimed by $\mathrm{OH}$; and

- a bad overall RPC time-of-flight resolution of 305 ps and an apparent advance of the timing signal of protons with respect to that of pions by $\sim 500$ ps ('500 ps effect').

These three problems, together with a number of additional mistakes [26, 27], have the following fatal consequences for cross-sections of secondary hadron production:

- cross-section spectra of secondary hadrons are distorted especially in regions where cross-sections vary strongly with momentum; and

- protons and pions are partly confused.

Discussions of the flaws in the $\mathrm{OH}$ analysis have been published in [3] and [4]. Criticisms of the $\mathrm{OH}$ analysis by independent review bodies can be found in $[9,10]$. We summarize here the main points.

OH's argument that 'dynamic' TPC track distortions can be neglected during the first third of the $400 \mathrm{~ms}$ long accelerator spill [8], reads as "... owing to their limited mobility the first [argon] ions created in the amplification region need about $25 \mathrm{~ms}$ to reach the drift region and subsequently the steady flow of ions into this region only starts approximately $100 \mathrm{~ms}$ after the start of the spill, with a gradual transition between these two regimes..." This argument is wrong. With an electric field strength of $\sim 1.7 \mathrm{kV} / \mathrm{cm}$ the argon ions need less than $1 \mathrm{~ms}$ for the relevant distance of $11 \mathrm{~mm}$. Therefore, dynamic TPC track distortions increase right away approximately linearly with time in the spill.
(Dynamic track distortions in the HARP TPC attain in the $r \cdot \phi$ coordinate $\sim 10 \mathrm{~mm}$ at the end of the spill, one order of magnitude larger than the typical $r \cdot \phi$ resolution; the correct algorithms to cope with TPC track distortions are described in [1] and in ample detail in [28-31].)

OH's claims in [8] that "...The constrained fit [which uses the beam point in addition to the TPC clusters] is unbiased with respect to the unconstrained fit [which uses the TPC clusters only]..." and "...The weight of the vertex constraint compensates very well the distortions..." contradict logic. It is impossible that a circle fit of TPC clusters that shifted away from their nominal $r \cdot \phi$ positions, together with the (undistorted) beam point, yields the same $p_{\mathrm{T}}$ as a fit of the distorted TPC clusters alone. It is equally impossible that a circle fit of the distorted TPC clusters together with the undistorted beam point gives an unbiased $p_{\mathrm{T}}$ estimate.

OH's justification of their claim of the absence of a bias in their circle fit stems from Fig. 4 in [8]: “...In Fig. 4 it is shown that the vertex constraint does not introduce biases for those particle trajectories and that the simulation provides an excellent description of the behaviour of the resolution function...". Their Fig. 4 claims to prove the equality of momentum from OH's 'constrained' and 'unconstrained fits'. That this claim is wrong is evident from the unphysical non-Gaussian shape of the shown distribution. Its cause is a mistake in their calculation of the $r \cdot \phi$ error of TPC clusters: their $\sigma_{r \phi}^{2}$ is multiplied by a factor $\cos ^{2} 2 \phi$ which assigns clusters an unphysically large weight depending on how close they are to the singular values $\phi=45^{\circ}, 135^{\circ}$, $225^{\circ}$ and $315^{\circ}$ in the azimuthal angle. (The mathematical intricacies of this mistake are explained in [26].)

$\mathrm{OH}$ never presented evidence that their $p_{\mathrm{T}}$ resolution during the accelerator spill is indeed $\sigma\left(1 / p_{\mathrm{T}}\right) \simeq$ $0.30(\mathrm{GeV} / c)^{-1}$, and that after TPC track distortion corrections their $r \cdot \phi$ residuals with respect to an unbiased external coordinated system are compatible with zero across the whole active TPC volume.

In their most recent physics publication [25], $\mathrm{OH}$ claim "...Corrections that allow use of the full statistics to be made, correcting for such [dynamic] distortions, have been developed... and are fully applied in this analysis. The obtained results are fully compatible within the statistical errors and differential systematic uncertainties with those previously published...". This claimed agreement between data from the first third of the spill without distortion correction, with data from the full spill with distortion correction, permits the conclusion that OH's full-spill analysis is beset by the same flaws as their earlier analysis of data from the first third of the spill.

Since $\mathrm{OH}$ have a biased track momentum, they observe that the RPC timing signal of protons is advanced with respect to the RPC timing signal of (relativistic) pions. This '500 ps effect' observation led them to conclude in [35] 
“...While this is in itself an interesting effect ...it prevents the use of the RPCs as a method to verify the reconstructed momentum scale of heavily ionizing particles [protons]." As a consequence, they made no use of the powerful particle identification capability from RPC time of flight. The exclusive use of $\mathrm{d} E / \mathrm{d} x$ from the TPC in conjunction with a biased track momentum leads to the partial confusion of protons and pions in $\mathrm{OH}$ 's analysis.

OH's interpretation of the ' $500 \mathrm{ps}$ effect' is characterized by statements like "... One possible explanation is the fluctuation in arrival time of the first cluster of the primary ionization. This fluctuation is smaller for heavily ionizing particles [protons]..." in [7], or "...An order of magnitude estimate of the effect given the propagation velocity of electrons in the gas and the chamber gap leads to an order of magnitude of a few 100 ps..." in [35]. This understanding of signal generation is wrong. The anode signal is generated by induction. Hence the (fast) propagation of electromagnetic waves across the gas gap is relevant and not the arrival at the anode of - in comparison-slowly moving electrons. (The correct mechanism of RPC-signal generation is described in [2].)

We conclude that claims and results published by $\mathrm{OH}$ [5-8, 25, 32-34, 36-38] suffer from systematic biases and shortcomings that are absent in our analyses presented in this and forthcoming papers.

\section{References}

1. V. Ammosov et al., Nucl. Instrum. Methods Phys. Res. A 588, 294 (2008)

2. V. Ammosov et al., Nucl. Instrum. Methods Phys. Res. A 578, 119 (2007)

3. V. Ammosov et al., J. Instrum. 3, P01002 (2008)

4. V. Ammosov et al., Eur. Phys. J. C 54, 169 (2008)

5. M.G. Catanesi et al., Nucl. Instrum. Methods Phys. Res. A 571, 527 (2007)

6. M. Bogomilov et al., IEEE Trans. Nucl. Sci. 54, 342 (2007)

7. A. Artamonov et al., J. Instrum. 2, P10004 (2007)

8. M.G. Catanesi et al., J. Instrum. 3, P04007 (2008)

9. Report on HARP Data comparisons, CERN-SPSC-2009-004 (SPSC-M-768)

10. Minutes of the SPSC Meetings, http://cern.ch/Committees/ SPSC/datesSPSCminutes.html
11. V. Ammosov et al., CERN-HARP-CDP-2008-001 (HARP Memo 08-101)

12. V. Ammosov et al., CERN-HARP-CDP-2008-002 (HARP Memo 08-102)

13. K. Fujii et al., Nucl. Instrum. Methods Phys. Res. A 264, 297 (1988)

14. F. Dydak, Yu. Nefedov, CERN-HARP-CDP-2004-001 (HARP Memo 04-002)

15. K. Fujii et al., Track reconstruction with the TRISTAN-TOPAZ TPC, KEK preprint 87-24; JLC Physics Group, Introduction to helical track manipulations (Internal Report, 6 June 1997)

16. V. Ammosov et al., CERN-HARP-CDP-2006-005 (HARP Memo 06-103)

17. N.I. Chernov, G.A. Ososkov, Comput. Phys. Commun. 33, 329 (1984)

18. S. Agostinelli et al., Nucl. Instrum. Methods Phys. Res. A 506, 250 (2003)

19. J. Allison et al., IEEE Trans. Nucl. Sci. 53, 270 (2006)

20. A. Bolshakova et al., Eur. Phys. J. C 56, 323 (2008)

21. A. Bolshakova et al., Tables of cross-sections of large-angle hadron production in proton- and pion-nucleus interactions I: beryllium nuclei and beam momenta of $+8.9 \mathrm{GeV} / c$ and $-8.0 \mathrm{GeV} / c$, CERN-HARP-CDP-2009-001

22. A. Bolshakova et al., Cross-sections of large-angle hadron production in proton- and pion-nucleus interactions II: beryllium nuclei and beam momenta from $\pm 3 \mathrm{GeV} / c$ to $\pm 15 \mathrm{GeV} / c$. Eur. Phys. J. C (2009, submitted)

23. T. Abbott et al., Phys. Rev. D 45, 3906 (1992)

24. I. Chemakin et al., Phys. Rev. C 65, 024904 (2002)

25. M.G. Catanesi et al., Phys. Rev. C 77, 055207 (2008)

26. V. Ammosov et al., CERN-HARP-CDP-2006-003 (HARP Memo 06-101)

27. V. Ammosov et al., CERN-HARP-CDP-2006-007 (HARP Memo 06-105) and CERN-HARP-CDP-2007-001 (HARP Memo 07-101)

28. F. Dydak, CERN-HARP-CDP-2003-001 (HARP Memo 03-001)

29. F. Dydak, A. Krasnoperov, Yu. Nefedov, CERN-HARP-CDP2003-002 (HARP Memo 03-002)

30. I. Boyko et al., CERN-HARP-CDP-2005-001 (HARP Memo 05-101)

31. V. Ammosov et al., CERN-HARP-CDP-2007-003 (HARP Memo 07-102)

32. M.G. Catanesi et al., Eur. Phys. J. C 51, 787 (2007)

33. M.G. Catanesi et al., Eur. Phys. J. C 53, 177 (2008)

34. M.G. Catanesi et al., Eur. Phys. J. C 54, 37 (2008)

35. HARP Collaboration, arXiv:0709.2806v1 [physics.ins-det]

36. HARP Collaboration, Nucl. Instrum. Methods Phys. Res. A 571, 564 (2007)

37. M. Bogomilov et al., IEEE Trans. Nucl. Sci. 54, 1455 (2007)

38. HARP Collaboration, Nucl. Instrum. Methods Phys. Res. A 588, 318 (2008) 